\title{
STARK UNITS IN POSITIVE CHARACTERISTIC
}

\author{
BRUNO ANGLÈS, TUAN NGO DAC, AND FLORIC TAVARES RIBEIRO
}

\begin{abstract}
We show that the module of Stark units associated to a signnormalized rank one Drinfeld module can be obtained from Anderson's equivariant $A$-harmonic series. We apply this to obtain a class formula à la Taelman and to prove a several variable log-algebraicity theorem, generalizing Anderson's log-algebraicity theorem. We also give another proof of Anderson's log-algebraicity theorem using shtukas and obtain various results concerning the module of Stark units for Drinfeld modules of arbitrary rank.
\end{abstract}

\section{Contents}

Introduction

1. Notation

2. Stark units and $L$-series attached to Drinfeld modules

2.1. $L$-series attached to Drinfeld modules

2.2. Stark units and the Taelman class module

2.3. Co-volumes

2.4. Regulator of Stark units and $L$-series

3. Stark units associated to sign-normalized rank one Drinfeld modules

3.1. Zeta functions

3.2. Background on sign-normalized rank one Drinfeld modules 18

3.3. Equivariant $A$-harmonic series: a detailed example 21

3.4. Stark units 24

3.5. A class formula à la Taelman 30

4. Log-Algebraicity Theorem 32

4.1. A refinement of Anderson's log-algebraicity theorem 32

4.2. Several variable $L$-series and shtukas 35

4.3. Stark units and several variable log-algebraicity theorem 4

4.4. Another proof of Anderson's log-algebraicity theorem

References 47

Date: September 25, 2018.

2010 Mathematics Subject Classification. Primary 11G09; Secondary 11M38, 11R58.

Key words and phrases. Stark units, $L$-series in positive characteristics, class formula, logalgebraicity theorem, shtukas.

${ }^{1}$ The second author was partially supported by ANR Grant PerCoLaTor ANR-14-CE25-0002. 


\section{INTRODUCTION}

The power-series $\sum_{n \geq 1} \frac{z^{n}}{n}$ is log-algebraic:

$$
\sum_{n \geq 1} \frac{z^{n}}{n}=-\log (1-z) .
$$

This identity allows one to obtain the value of a Dirichlet $L$-series at $s=1$ as an algebraic linear combination of logarithms of circular units. Inspired by examples of Carlitz [10, and Thakur [26, Anderson (11, 2]) proved an analogue of this identity for a sign-normalized rank one Drinfeld $A$-module, known as Anderson's $\log$-algebraicity theorem.

When $A=\mathbb{F}_{q}[\theta]$ (the genus 0 case), various works (1, 2, 3, 6, 7, 9, 11, 15, 16, 17, $20,21,22,23$ ) have revealed the importance of certain units in the study of special values of the Goss $L$-functions at $s=1$. To give a simple example, the Carlitz module is considered to play the role of the multiplicative group $\mathbb{G}_{m}$ over $\mathbb{Z}$, and Anderson ([1, 2]) showed that the images through the Carlitz exponential of some special units give algebraic elements which are the equivalent of the circular units. The special units constructed in such a way are then "log-algebraic". Recently, Taelman $(22,23])$ introduced the module of units attached to any Drinfeld module and proved a class formula which states that the special value of the Goss $L$ function attached to a Drinfeld module at $s=1$ is the product of a regulator term arising from the module of units and an algebraic term arising from a certain class module. Also, deformations of Goss $L$-series values in Tate algebras are investigated by Pellarin and two of the authors $([6,7,9,21])$. For higher dimensional versions of Drinfeld modules, we refer the reader to [3, 11, 15, 16, 17, 20]. We should mention that all these works are based on a crucial fact that $\mathbb{F}_{q}[\theta]$ is a principal ideal domain, which is no longer true in general.

In the present paper, we develop a new method to deal with higher genus cases. We introduce Stark units attached to Drinfeld $A$-modules extending the previous work of two of the authors (9]) and make a systematic study of these modules of Stark units. For a sign-normalized rank one Drinfeld module, we prove a direct link between the module of Stark units and Anderson's equivariant $A$-harmonic series, which is an analogue of Stark's conjectures. It allows us to obtain a class formula à la Taelman and a several variable log-algebraicity theorem in the general context.

Let us give now more precise statements of our results.

Let $K / \mathbb{F}_{q}$ be a global function field $\left(\mathbb{F}_{q}\right.$ is algebraically closed in $K$ ), let $A$ be the ring of elements of $K$ which are regular outside a fixed place $\infty$ of $K$ of degree $d_{\infty} \geq 1$. The completion $K_{\infty}$ of $K$ at the place $\infty$ has residue field $\mathbb{F}_{\infty}$ and is endowed with the $\infty$-adic valuation $v_{\infty}: K_{\infty} \rightarrow \mathbb{Z} \cup\{+\infty\}$. For $a \in A$, we set: $\operatorname{deg} a:=-d_{\infty} v_{\infty}(a)$. We fix an algebraic closure $\bar{K}_{\infty}$ of $K_{\infty}$, and still denote $v_{\infty}: \bar{K}_{\infty} \rightarrow \mathbb{Q} \cup\{+\infty\}$ the extension of $v_{\infty}$ to $\bar{K}_{\infty}$. Let $\tau: \bar{K}_{\infty} \rightarrow \bar{K}_{\infty}$ be the $\mathbb{F}_{q}$-algebra homomorphism which sends $x$ to $x^{q}$.

We choose a sign function sgn : $K_{\infty}^{\times} \rightarrow \mathbb{F}_{\infty}^{\times}$, that is, a group homomorphism such that sgn $\left.\right|_{\mathbb{F}_{\infty}^{\times}}=\operatorname{Id}_{\mathbb{F}_{\infty}^{\times}}$. Let $\phi: A \hookrightarrow \bar{K}_{\infty}\{\tau\}$ be a sign-normalized rank one Drinfeld module (see Section 3.2), i.e. there exists an integer $i(\phi) \in \mathbb{N}$ such that:

$$
\forall a \in A, \quad \phi_{a}=a+\cdots+\operatorname{sgn}(a)^{q^{i(\phi)}} \tau^{\operatorname{deg} a} .
$$


Then, the exponential series attached to $\phi$ is the unique element $\exp _{\phi} \in \bar{K}_{\infty}\{\{\tau\}\}$, such that $\exp _{\phi} \equiv 1(\bmod \tau)$, and:

$$
\forall a \in A, \quad \exp _{\phi} a=\phi_{a} \exp _{\phi} .
$$

If we write:

$$
\exp _{\phi}=\sum_{i \geq 0} e_{i}(\phi) \tau^{i}
$$

with $e_{i}(\phi) \in \bar{K}_{\infty}$, then the field $H:=K\left(e_{i}(\phi), i \in \mathbb{N}\right)$ is a finite abelian extension of $K$ which is unramified outside $\infty$ (see Section 3.2). Let $B$ be the integral closure of $A$ in $H$. For all $a \in A$, we have:

$$
\phi_{a} \in B\{\tau\} .
$$

For a non-zero ideal $I$ of $A$, we define $\phi_{I} \in H\{\tau\}$ to be the monic element in $H\{\tau\}$ such that:

$$
H\{\tau\} \phi_{I}=\sum_{a \in I} H\{\tau\} \phi_{a} .
$$

In fact, $\phi_{I} \in B\{\tau\}$ and we denote its constant term by $\psi(I) \in B \backslash\{0\}$.

For simplicity, we will work over the abelian extension $H / K$. We should mention that the results presented below are still valid for any finite abelian extension $E / K$ such that $H \subset E$.

Let $G=\operatorname{Gal}(H / K)$. For a non-zero ideal $I$ of $A$, we denote by $\sigma_{I}=(I, H / K) \in$ $G$, where $(\cdot, H / K)$ is the Artin map. Let $z$ be an indeterminate over $K_{\infty}$ and let $\mathbb{T}_{z}\left(K_{\infty}\right)$ be the Tate algebra in the variable $z$ with coefficients in $K_{\infty}$. Let's set:

$$
H_{\infty}=H \otimes_{K} K_{\infty},
$$

and:

$$
\mathbb{T}_{z}\left(H_{\infty}\right)=H \otimes_{K} \mathbb{T}_{z}\left(K_{\infty}\right) .
$$

Let $\tau: \mathbb{T}_{z}\left(H_{\infty}\right) \rightarrow \mathbb{T}_{z}\left(H_{\infty}\right)$ be the continuous $\mathbb{F}_{q}[z]$-algebra homomorphism such that:

$$
\forall x \in H_{\infty}, \quad \tau(x)=x^{q} .
$$

We set:

$$
\exp _{\widetilde{\phi}}=\sum_{i \geq 0} e_{i}(\phi) z^{i} \tau^{i} \in H[z]\{\{\tau\}\} .
$$

Then $\exp _{\tilde{\phi}}$ converges on $\mathbb{T}_{z}\left(H_{\infty}\right)$. Following [9], we introduce the module of $z$-units attached to $\phi / B$ :

$$
U(\widetilde{\phi} / B[z])=\left\{f \in \mathbb{T}_{z}\left(H_{\infty}\right), \exp _{\widetilde{\phi}}(f) \in B[z]\right\} .
$$

We denote by ev : $\mathbb{T}_{z}\left(H_{\infty}\right) \rightarrow H_{\infty}$ the evaluation at $z=1$. The module of Stark units attached to $\phi / B$ is defined by (see [9], Section 2 ):

$$
U_{S t}(\phi / B)=\operatorname{ev}(U(\widetilde{\phi} / B[z])) \subset H_{\infty} .
$$

Then $U_{S t}(\phi / B)$ is an $A$-lattice in $H_{\infty}$ (see Theorem 2.7), i.e. $U_{S t}(\phi / B)$ is an $A$ module which is discrete and cocompact in $H_{\infty}$. In fact, $U_{S t}(\phi / B)$ is contained in the $A$-module of the Taelman module of units [22] defined by:

$$
U(\phi / B)=\left\{x \in H_{\infty}, \exp _{\phi}(x) \in B\right\},
$$


which is also an $A$-lattice in $H_{\infty}$. Following Taelman [22, the Taelman class module $H(\phi / B)$ is a finite $A$-module (via $\phi$ ) defined by:

$$
H(\phi / B)=\frac{H_{\infty}}{B+\exp _{\phi}\left(H_{\infty}\right)}
$$

Following Anderson [1, we introduce the following series (see Section 3.3):

$$
\mathcal{L}(\phi / B ; 1 ; z)=\sum_{I} \frac{z^{\operatorname{deg} I}}{\psi(I)} \sigma_{I} \in \mathbb{T}_{z}\left(H_{\infty}\right)[G]
$$

where the sum runs through the non-zero ideals $I$ of $A$. The equivariant $A$-harmonic series attached to $\phi / B$ is defined by:

$$
\mathcal{L}(\phi / B)=\operatorname{ev}(\mathcal{L}(\phi / B ; 1 ; z)) \in H_{\infty}[G] .
$$

One of our main theorems states (see Theorem 3.8) that the module of Stark units $U_{S t}(\phi / B)$ can be obtained from the equivariant $A$-harmonic series $\mathcal{L}(\phi / B)$, which is reminiscent of Stark's Conjectures ([25]):

Theorem A. We have:

$$
U(\widetilde{\phi} / B[z])=\mathcal{L}(\phi / B ; 1 ; z) B[z] .
$$

In particular,

$$
U_{S t}(\phi / B)=\mathcal{L}(\phi / B) B .
$$

We will present several applications of this theorem.

Firstly, we apply Theorem $\AA$ to obtain a class formula à la Taelman for $\phi / B$, by a different method of Taelman's original one 23 . Roughly speaking, we introduce the Stark regulator (resp. the regulator defined by Taelman [23]) attached to $\phi / B$ by $\left[B: U_{S t}(\phi / B)\right]_{A} \in \bar{K}_{\infty}^{\times}$(resp. $\left.[B: U(\phi / B)]_{A} \in \bar{K}_{\infty}^{\times}\right)$(see Section 2.3). We show (see Theorem 2.7):

Theorem B. We have:

$$
\operatorname{Fitt}_{A} \frac{U(\phi / B)}{U_{S t}(\phi / B)}=\operatorname{Fitt}_{A} H(\phi / B)
$$

where, for a finite $A$-module $M$, Fitt $_{A} M$ is the Fitting ideal of $M$.

Observe that $\mathcal{L}(\phi / B)$ induces a $K_{\infty}$-linear map on $H_{\infty}$, and we denote by $\operatorname{det}_{K_{\infty}} \mathcal{L}(\phi / B)$ its determinant. We prove the following formula (see Theorem 3.6):

$$
\operatorname{det}_{K_{\infty}} \mathcal{L}(\phi / B)=\zeta_{B}(1):=\prod_{\mathfrak{P}}\left(1-\frac{1}{\left[\frac{B}{\mathfrak{P}}\right]_{A}}\right)^{-1} \in \bar{K}_{\infty}^{\times},
$$

where $\mathfrak{P}$ runs through the maximal ideals of $B$. Note that $\zeta_{B}(1)$ is a special value at $s=1$ of some zeta function $\zeta_{B}(s)$ introduced by Goss (see [19], Chapter 8). Therefore, Theorem $\mathrm{A}$ and Theorem $\mathrm{B}$ imply Taelman's class formula for $\phi / B$ (see Theorem 3.10):

Theorem C. We have:

$$
\zeta_{B}(1)=\left[B: U_{S t}(\phi / B)\right]_{A}=[B: U(\phi / B)]_{A}[H(\phi / B)]_{A} .
$$


When the genus of $K$ is zero and $d_{\infty}=1$, Taelman's class formula, its higher dimensional versions, and its arithmetic consequences are now well-understood due

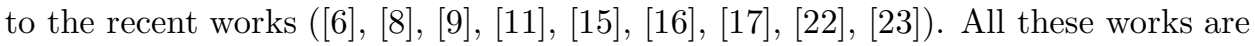
based on the crucial fact that when $g=0$ and $d_{\infty}=1$, the ring $A$ is a principal ideal domain (when $A$ is not assumed to be principal, the existence of a class formula is still an open problem in general). Using the module of Stark units, we are able to overcome this difficulty, and Theorem $\mathrm{C}$ provides a large class of examples of Taelman's class formula when $A$ is no longer principal. We refer the reader to Section 2.4 for a more detailed discussion.

Secondly, we apply Theorem $\mathrm{A}$ to prove a several variable log-algebraicity theorem, generalizing Anderson's log-algebraicity theorems (see Theorem 4.2). (The theorem below is valid for any finite abelian extension $E / K, H \subset E$, see Theorem 4.2 for the precise statement).

Theorem D. Let $n \geq 0$ and let $X_{1}, \ldots, X_{n}, z$ be $n+1$ indeterminates over $K$. Let $\tau: K\left[X_{1}, \ldots, X_{n}\right][[z]] \rightarrow K\left[X_{1}, \ldots, X_{n}\right][[z]]$ be the continuous $\mathbb{F}_{q}[[z]]$-algebra homomorphism for the $z$-adic topology such that $\forall x \in K\left[X_{1}, \ldots, X_{n}\right], \tau(x)=x^{q}$. Then:

$$
\forall b \in B, \quad \exp _{\widetilde{\phi}}\left(\sum_{I} \frac{\sigma_{I}(b)}{\psi(I)} \phi_{I}\left(X_{1}\right) \cdots \phi_{I}\left(X_{n}\right) z^{\operatorname{deg} I}\right) \in B\left[X_{1}, \ldots, X_{n}, z\right],
$$

where $I$ runs through the non-zero ideals of $A$.

For $n \leq 1$ and $d_{\infty}=1$, this theorem was due to G. Anderson ([1], Theorem 5.1.1 and 2, Theorem 3):

$$
\begin{gathered}
\forall b \in B, \quad \exp _{\widetilde{\phi}}\left(\sum_{I} \frac{\sigma_{I}(b)}{\psi(I)} z^{\operatorname{deg} I}\right) \in B[z], \\
\forall b \in B, \quad \exp _{\widetilde{\phi}}\left(\sum_{I} \frac{\sigma_{I}(b)}{\psi(I)} \phi_{I}(X) z^{\operatorname{deg} I}\right) \in B[X, z],
\end{gathered}
$$

where the sum runs through the non-zero ideals of $A$. Again, this result is now well-understood when the genus of $K$ is zero (and $d_{\infty}=1$ ) due to the recent works of many people $(6], 7], 8], 9], 23$, 28] Sections 8.9 and 8.10, and the forthcoming work of M. Papanikolas [20]). However, to our knowledge, Anderson's $\log$-algebraicity remains quite mysterious for $g>0$ until now.

Thirdly, we present an alternative approach to the previous several variable logalgebraicity theorem (Theorem D) via Drinfeld's correspondence between Drinfeld modules and shtukas. Using the shtuka function attached to $\phi / B$ via Drinfeld's correspondence, we introduce one variable versions of the previous objects, i.e. the modules of $z$-units and Stark units, the equivariant $A$-harmonic series and the $L$ series (see Section 4.2). We prove an analogue of Theorem $\mathrm{A}$ in this one variable context (see Theorem 4.9). More generally, we also obtain a several variable logalgebraicity theorem (see Corollary 4.10). In the case $g=0$ and $d_{\infty}=1$, we rediscover the Pellarin's $L$-series [21] and its several variable variants studied in [4, [6], 7], 9. We deduce from this another proof of Theorem D (see Section 4.4).

Finally, we prove some results concerning the module of Stark units for Drinfeld modules of arbitrary rank in Section 2. In particular, Theorem B is still valid for any Drinfeld module. 


\section{Notation}

Let $K / \mathbb{F}_{q}$ be a global function field of genus $g$, where $\mathbb{F}_{q}$ is a finite field of characteristic $p$, having $q$ elements $\left(\mathbb{F}_{q}\right.$ is algebraically closed in $\left.K\right)$. We fix a place $\infty$ of $K$ of degree $d_{\infty}$, and denote by $A$ the ring of elements of $K$ which are regular outside of $\infty$. The completion $K_{\infty}$ of $K$ at the place $\infty$ has residue field $\mathbb{F}_{\infty}$ and comes with the $\infty$-adic valuation $v_{\infty}: K_{\infty} \rightarrow \mathbb{Z} \cup\{+\infty\}$. We fix an algebraic closure $\bar{K}_{\infty}$ of $K_{\infty}$ and still denote by $v_{\infty}: \mathbb{C}_{\infty} \rightarrow \mathbb{Q} \cup\{+\infty\}$ the extension of $v_{\infty}$ to the completion $\mathbb{C}_{\infty}$ of $\bar{K}_{\infty}$.

We will fix a uniformizer $\pi$ of $K_{\infty}$. Set $\pi_{1}=\pi$, and for $n \geq 2$, choose $\pi_{n} \in \bar{K}_{\infty}^{\times}$ such that $\pi_{n}^{n}=\pi_{n-1}$. If $z \in \mathbb{Q}, z=\frac{m}{n !}$ for some $m \in \mathbb{Z}, n \geq 1$, we set:

$$
\pi^{z}:=\pi_{n}^{m} \text {. }
$$

Let $\overline{\mathbb{F}}_{q}$ be the algebraic closure of $\mathbb{F}_{q}$ in $\bar{K}_{\infty}$, and let $U_{\infty}=\left\{x \in \bar{K}_{\infty}, v_{\infty}(x-1)>0\right\}$. Then:

$$
\bar{K}_{\infty}^{\times}=\pi^{\mathbb{Q}} \times \overline{\mathbb{F}}_{q}^{\times} \times U_{\infty} .
$$

Therefore, if $x \in \bar{K}_{\infty}^{\times}$, one can write in a unique way:

$$
x=\pi^{v_{\infty}(x)} \operatorname{sgn}(x)\langle x\rangle, \quad \operatorname{sgn}(x) \in \overline{\mathbb{F}}_{q}^{\times},\langle x\rangle \in U_{\infty} .
$$

Let $\mathcal{I}(A)$ be the group of non-zero fractional ideals of $A$. For $I \in \mathcal{I}(A), I \subset A$, we set:

$$
\operatorname{deg} I:=\operatorname{dim}_{\mathbb{F}_{q}} A / I \text {. }
$$

Then, the function deg on non-zero ideals of $A$ extends into a group homomorphism:

$$
\operatorname{deg}: \mathcal{I}(A) \rightarrow \mathbb{Z}
$$

Let's observe that, for $x \in K^{\times}$, we have:

$$
\operatorname{deg}(x):=\operatorname{deg}(x A)=-d_{\infty} v_{\infty}(x) .
$$

Let $I \in \mathcal{I}(A)$, then there exists an integer $h \geq 1$ such that $I^{h}=x A, x \in K^{\times}$. We set:

$$
\langle I\rangle:=\langle x\rangle^{\frac{1}{h}} \in U_{\infty} .
$$

Then one shows (see [19], Section 8.2) that the map [.] $: \mathcal{I}(A) \rightarrow \bar{K}_{\infty}^{\times}, I \mapsto\langle I\rangle \pi^{-\frac{\operatorname{deg} I}{d_{\infty}}}$ is a group homomorphism such that:

$$
\forall x \in K^{\times}, \quad[x A]=\frac{x}{\operatorname{sgn}(x)} .
$$

Observe that:

$$
\forall I \in \mathcal{I}(A), \quad \operatorname{sgn}([I])=1 .
$$

If $M$ is a finite $A$-module, and $\operatorname{Fitt}_{A}(M)$ is the Fitting ideal of $M$, we set:

$$
[M]_{A}:=\left[\operatorname{Fitt}_{A}(M)\right] \text {. }
$$

Let's observe that, if $0 \rightarrow M_{1} \rightarrow M \rightarrow M_{2} \rightarrow 0$ is a short exact sequence of finite $A$-modules, then:

$$
[M]_{A}=\left[M_{1}\right]_{A}\left[M_{2}\right]_{A} .
$$

Let $E / K$ be a finite extension, and let $O_{E}$ be the integral closure of $A$ in $E$. Let $\mathcal{I}\left(O_{E}\right)$ be the group of non-zero fractional ideals of $O_{E}$. We denote by $N_{E / K}$ : 
$\mathcal{I}\left(O_{E}\right) \rightarrow \mathcal{I}(A)$ the group homomorphism such that, if $\mathfrak{P}$ is a maximal ideal of $O_{E}$ and $P=\mathfrak{P} \cap A$, we have:

$$
N_{E / K}(\mathfrak{P})=P^{\left[\frac{O_{E}}{\mathfrak{P}}: \frac{A}{P}\right]} .
$$

Note that, if $\mathfrak{P}=x O_{E}, x \in E^{\times}$, then:

$$
N_{E / K}(\mathfrak{P})=N_{E / K}(x) A,
$$

where $N_{E / K}: E \rightarrow K$ also denotes the usual norm map.

\section{Stark units and $L$-Series attached to Drinfeld modules}

\section{1. $L$-series attached to Drinfeld modules.}

Let $E / K$ be a finite extension, and let $O_{E}$ be the integral closure of $A$ in $E$. Let $\tau: E \rightarrow E, x \mapsto x^{q}$. Let $\rho$ be an Drinfeld $A$-module (or a Drinfeld module for short) of rank $r \geq 1$ defined over $O_{E}$, i.e. $\rho: A \hookrightarrow O_{E}\{\tau\}$ is an $\mathbb{F}_{q}$-algebra homomorphism such that:

$$
\forall a \in A \backslash\{0\}, \quad \rho_{a}=\rho_{a, 0}+\rho_{a, 1} \tau+\cdots+\rho_{a, r \operatorname{deg} a} \tau^{r \operatorname{deg} a},
$$

where $\rho_{a, 0}, \ldots, \rho_{a, r \operatorname{deg} a} \in O_{E}, \rho_{a, 0}=a$, and $\rho_{a, r \operatorname{deg} a} \neq 0$.

Let $\mathfrak{P}$ be a maximal ideal of $O_{E}$, we denote by $\rho\left(O_{E} / \mathfrak{P}\right)$ the finite dimensional

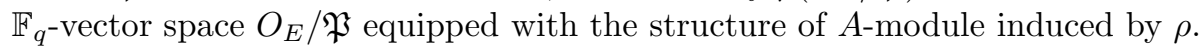

Proposition 2.1. The following product converges to a principal unit in $K_{\infty}^{\times}$(i.e. an element in $\left.U_{\infty} \cap K_{\infty}^{\times}\right)$:

$$
L_{A}\left(\rho / O_{E}\right):=\prod_{\mathfrak{P}} \frac{\left[\frac{O_{E}}{\mathfrak{P}}\right]_{A}}{\left[\rho\left(\frac{O_{E}}{\mathfrak{P}}\right)\right]_{A}},
$$

where $\mathfrak{P}$ runs through the maximal ideals of $O_{E}$.

Proof. By [19], Remark 7.1.8.2, we have: $H_{A} \subset E$, where $H_{A} / K$ is the maximal unramified abelian extension of $K$ such that $\infty$ splits completely in $H_{A}$. Thus $N_{E / K}(\mathfrak{P})$ is a principal ideal. Observe that:

$$
\operatorname{Fitt}_{A} \frac{O_{E}}{\mathfrak{P}}=N_{E / K}(\mathfrak{P}) .
$$

Thus:

$$
\left[\frac{O_{E}}{\mathfrak{P}}\right]_{A}=\left[N_{E / K}(\mathfrak{P})\right] .
$$

By [18, Theorem 5.1, there exists a unitary polynomial $P(X) \in A[X]$ of degree $r^{\prime} \leq r$ such that:

$$
\begin{aligned}
N_{E / K}(\mathfrak{P}) & =P(0) A, \\
\operatorname{Fitt}_{A} \rho\left(\frac{O_{E}}{\mathfrak{P}}\right) & =P(1) A, \\
v_{\infty}\left(\frac{(-1)^{r^{\prime}} P(0)}{P(1)}-1\right) & \geq \frac{\operatorname{deg}\left(N_{E / K}(\mathfrak{P})\right)}{r^{\prime} d_{\infty}} .
\end{aligned}
$$

This last assertion comes from the fact that $P(X)$ is a power of the minimal polynomial over $K$ of the Frobenius $F$ of $\frac{O_{E}}{\mathfrak{P}}$ (see [18, Lemma 3.3), and that $K(F) / K$ 
is totally imaginary (i.e. there exists a unique place of $K(F)$ over $\infty$ ). By the properties of [.] (see Section 11), we have:

$$
\frac{\left[\frac{O_{E}}{\mathfrak{P}}\right]_{A}}{\left[\rho\left(\frac{O_{E}}{\mathfrak{P}^{\prime}}\right)\right]_{A}}=\frac{(-1)^{r^{\prime}} P(0)}{P(1)} .
$$

The proposition follows.

Remark 2.2. The element $L_{A}\left(\rho / O_{E}\right) \in K_{\infty}^{\times}$is called the $L$-series attached to $\rho / O_{E}$. By the proof of Proposition 2.1. $L_{A}\left(\rho / O_{E}\right)$ depends on $A, \rho$ and $O_{E}$, but not on the choice of $\pi$.

Let $F / K$ be a finite extension with $F \subset E$, and such that there exists a unique place of $F$ above $\infty$ (still denoted by $\infty$ ). Let $A^{\prime}$ be the integral closure of $A$ in $F$, then $A^{\prime}$ is the set of elements in $F$ which are regular outside $\infty$. We assume that $\rho$ extends into a Drinfeld $A^{\prime}$-module: $\rho: A^{\prime} \hookrightarrow O_{E}\{\tau\}$. Let $[\cdot]_{A^{\prime}}: \mathcal{I}\left(A^{\prime}\right) \rightarrow \bar{K}_{\infty}^{\times}$be the map constructed as in Section 1 with the help of the choice of a uniformizer $\pi^{\prime} \in F_{\infty}^{\times}$. Let $N_{F_{\infty} / K_{\infty}}: F_{\infty} \rightarrow K_{\infty}$ be the usual norm map.

Corollary 2.3. We have:

$$
N_{F_{\infty} / K_{\infty}}\left(L_{A^{\prime}}\left(\rho / O_{E}\right)\right)=L_{A}\left(\rho / O_{E}\right) .
$$

Proof. Recall that:

$$
L_{A^{\prime}}\left(\rho / O_{E}\right):=\prod_{\mathfrak{P}} \frac{\left[\frac{O_{E}}{\mathfrak{P}^{\mathfrak{P}}}\right]_{A^{\prime}}}{\left[\rho\left(\frac{O_{E}}{\mathfrak{P}}\right)\right]_{A^{\prime}}},
$$

where $\mathfrak{P}$ runs through the maximal ideals of $O_{E}$. Since $N_{F_{\infty} / K_{\infty}}$ is continuous, we get by the proof of Proposition 2.1.

$$
N_{F_{\infty} / K_{\infty}}\left(L_{A^{\prime}}\left(\rho / O_{E}\right)\right)=\prod_{\mathfrak{P}} N_{F_{\infty} / K_{\infty}}\left(\frac{\left[\frac{O_{E}}{\mathfrak{P}}\right]_{A^{\prime}}}{\left[\rho\left(\frac{O_{E}}{\mathfrak{P}}\right)\right]_{A^{\prime}}}\right) .
$$

Let $\mathfrak{P}$ be a maximal ideal of $O_{E}$. Since $\frac{\left[\frac{O_{E}}{\mathcal{F}}\right]_{A^{\prime}}}{\left[\rho\left(\frac{O_{E}}{\mathcal{P}}\right)\right]_{A^{\prime}}} \in F^{\times}$, we get:

$$
N_{F_{\infty} / K_{\infty}}\left(\frac{\left[\frac{O_{E}}{\mathfrak{P}^{3}}\right]_{A^{\prime}}}{\left[\rho\left(\frac{O_{E}}{\mathfrak{P}^{\mathfrak{P}}}\right)\right]_{A^{\prime}}}\right)=N_{F / K}\left(\frac{\left[\frac{O_{E}}{\mathfrak{P}^{\mathfrak{P}}}\right]_{A^{\prime}}}{\left[\rho\left(\frac{O_{E}}{\mathfrak{P}^{3}}\right)\right]_{A^{\prime}}}\right) .
$$

But, observe that if $M$ is a finite $A^{\prime}$-module, we have:

$$
N_{F / K}\left(\operatorname{Fitt}_{A^{\prime}} M\right)=\operatorname{Fitt}_{A} M \text {. }
$$

By the proof of Proposition 2.1] $\frac{\left[\frac{O_{E}}{\mathcal{F}_{F}}\right]_{A^{\prime}}}{\left[\rho\left(\frac{\mathcal{O}_{F}}{\mathcal{P}}\right)\right]_{A^{\prime}}}$ is a principal unit in $F_{\infty}^{\times}$, and therefore $N_{F / K}\left(\frac{\left[\frac{O_{E}}{\mathcal{F}}\right]_{A^{\prime}}}{\left[\rho\left(\frac{O_{E}}{\not 3}\right)\right]_{A^{\prime}}}\right)$ is also a principal unit in $K_{\infty}^{\times}$. Again, by the proof of Proposition 2.1, we get:

$$
N_{F / K}\left(\frac{\left[\frac{O_{E}}{\mathfrak{P}^{3}}\right]_{A^{\prime}}}{\left[\rho\left(\frac{O_{E}}{\mathfrak{P}^{\mathfrak{P}}}\right)\right]_{A^{\prime}}}\right)=\frac{\left[\frac{O_{E}}{\mathfrak{P}^{\prime}}\right]_{A}}{\left[\rho\left(\frac{O_{E}}{\mathfrak{P}^{3}}\right)\right]_{A}} .
$$

The corollary follows. 


\subsection{Stark units and the Taelman class module.}

Let $E / K$ be a finite extension of degree $n$, and let $O_{E}$ be the integral closure of $A$ in $E$. Set:

$$
E_{\infty}=E \otimes_{K} K_{\infty}
$$

Let $M$ be an $A$-module, $M \subset E_{\infty}$, we say that $M$ is an $A$-lattice in $E_{\infty}$ if $M$ is discrete and cocompact in $E_{\infty}$. Observe that if $M$ is an $A$-lattice in $E_{\infty}$, then there exist $e_{1}, \ldots, e_{n} \in E_{\infty}$ (recall that $n=[E: K]$ ) such that $E_{\infty}=\oplus_{i=1}^{n} K_{\infty} e_{i}$, $N:=\oplus_{i=1}^{n} A e_{i} \subset M$ and $\frac{M}{N}$ is a finite $A$-module. Note also that $O_{E}$ is an $A$-lattice in $E_{\infty}$.

Let $\tau: E_{\infty} \rightarrow E_{\infty}, x \mapsto x^{q}$. Let $\rho: A \hookrightarrow O_{E}\{\tau\}$ be a Drinfeld module of rank $r \geq 1$. Then, there exist unique elements $\exp _{\rho}, \log _{\rho} \in E\{\{\tau\}\}$ such that

$$
\begin{gathered}
\exp _{\rho}, \log _{\rho} \in 1+E\{\{\tau\}\} \tau, \\
\forall a \in A, \quad \exp _{\rho} a=\rho_{a} \exp _{\rho}, \\
\exp _{\rho} \log _{\rho}=\log _{\rho} \exp _{\rho}=1 .
\end{gathered}
$$

The formal series $\exp _{\rho}$, and $\log _{\rho}$ are respectively called the exponential series and the logarithm series associated to $\rho / O_{E}$. We will write:

$$
\begin{gathered}
\exp _{\rho}=\sum_{i \geq 0} e_{i}(\rho) \tau^{i}, \\
\log _{\rho}=\sum_{i \geq 0} l_{i}(\rho) \tau^{i},
\end{gathered}
$$

with $e_{i}(\rho), l_{i}(\rho) \in E$. Moreover, $\exp _{\rho}$ converges on $E_{\infty}$ (see [19], proof of Theorem 4.6.9).

Definition 2.4. We define the Taelman module of units associated to $\rho / O_{E}$ as follows:

$$
U\left(\rho / O_{E}\right)=\left\{x \in E_{\infty}, \exp _{\rho}(x) \in O_{E}\right\}
$$

Then, as a consequence of [22], Theorem 1, the $A$-module $U\left(\rho / O_{E}\right)$ is an $A$ lattice in $E_{\infty}$.

Definition 2.5. We define the Taelman class module associated to $\rho / O_{E}$ by:

$$
H\left(\rho / O_{E}\right)=\frac{E_{\infty}}{O_{E}+\exp _{\rho}\left(E_{\infty}\right)} .
$$

Note that $H\left(\rho / O_{E}\right)$ is an $A$-module via $\rho$, and by [22, Theorem $1, H\left(\rho / O_{E}\right)$ is a finite $A$-module.

Let $z$ be an indeterminate over $K_{\infty}$, and let $\mathbb{T}_{z}\left(K_{\infty}\right)$ be the Tate algebra in the variable $z$ with coefficients in $K_{\infty}$. We set:

$$
\mathbb{T}_{z}\left(E_{\infty}\right)=E \otimes_{K} \mathbb{T}_{z}\left(K_{\infty}\right)
$$

Observe that $E_{\infty} \subset \mathbb{T}_{z}\left(E_{\infty}\right)$, and $\mathbb{T}_{z}\left(E_{\infty}\right)$ is a free $\mathbb{T}_{z}\left(K_{\infty}\right)$-module of rank $[E: K]$. Let $\tau: \mathbb{T}_{z}\left(E_{\infty}\right) \rightarrow \mathbb{T}_{z}\left(E_{\infty}\right)$ be the continuous $\mathbb{F}_{q}[z]$-algebra homomorphism such that:

$$
\forall x \in E_{\infty}, \quad \tau(x)=x^{q} .
$$

Let ev $: \mathbb{T}_{z}\left(E_{\infty}\right) \rightarrow E_{\infty}$ be the surjective $E_{\infty}$-algebra homomorphism given by:

$$
\forall f \in \mathbb{T}_{z}\left(E_{\infty}\right), \quad \operatorname{ev}(f)=\left.f\right|_{z=1}
$$


We have: $\operatorname{ker}$ ev $=(z-1) \mathbb{T}_{z}\left(E_{\infty}\right)$, and:

$$
\forall f \in \mathbb{T}_{z}\left(E_{\infty}\right), \quad \operatorname{ev}(\tau(f))=\tau(\operatorname{ev}(f)) .
$$

Recall that:

$$
\exp _{\rho}=\sum_{i \geq 0} e_{i}(\rho) \tau^{i}, \quad \text { with } e_{i}(\rho) \in E
$$

We set:

$$
\exp _{\widetilde{\rho}}=\sum_{i \geq 0} e_{i}(\rho) z^{i} \tau^{i} \in E[z]\{\{\tau\}\} .
$$

Observe that $\exp _{\widetilde{\rho}}$ converges on $\mathbb{T}_{z}\left(E_{\infty}\right)$, and:

$$
\forall f \in \mathbb{T}_{z}\left(E_{\infty}\right), \quad \operatorname{ev}\left(\exp _{\widetilde{\rho}}(f)\right)=\exp _{\rho}(\operatorname{ev}(f)) .
$$

Let $\tilde{\rho}: A \hookrightarrow O_{E}[z]\{\tau\}$ be the $\mathbb{F}_{q}$-algebra homomorphism given by:

$$
\forall a \in A, \quad \widetilde{\rho}_{a}=a+\rho_{a, 1} z \tau+\cdots+\rho_{a, r \operatorname{deg} a} z^{r \operatorname{deg} a} \tau^{r \operatorname{deg} a},
$$

where $\rho_{a}=a+\rho_{a, 1} \tau+\cdots+\rho_{a, r \operatorname{deg} a} \tau^{r \operatorname{deg} a}$. Then:

$$
\forall a \in A, \quad \exp _{\widetilde{\rho}} a=\widetilde{\rho}_{a} \exp _{\widetilde{\rho}} .
$$

Definition 2.6. The module of $z$-units associated to $\rho / O_{E}$ is defined by:

$$
U\left(\widetilde{\rho} / O_{E}[z]\right)=\left\{f \in \mathbb{T}_{z}\left(E_{\infty}\right), \exp _{\widetilde{\rho}}(f) \in O_{E}[z]\right\} .
$$

And the module of Stark units associated to $\rho / O_{E}$ is defined by:

$$
U_{S t}\left(\rho / O_{E}\right):=\operatorname{ev}\left(U\left(\widetilde{\rho} / O_{E}[z]\right)\right) .
$$

Observe that $U_{S t}\left(\rho / O_{E}\right) \subset U\left(\rho / O_{E}\right)$.

Theorem 2.7. The A-module $U_{S t}\left(\rho / O_{E}\right)$ is an A-lattice in $E_{\infty}$. Furthermore:

$$
\left[\frac{U\left(\rho / O_{E}\right)}{U_{S t}\left(\rho / O_{E}\right)}\right]_{A}=\left[H\left(\rho / O_{E}\right)\right]_{A} .
$$

Proof. This is a consequence of the proof of [9, Theorem 1. For the convenience of the reader, we give a sketch of the proof. Let's set:

$$
H\left(\widetilde{\rho} / O_{E}[z]\right)=\frac{\mathbb{T}_{z}\left(E_{\infty}\right)}{O_{E}[z]+\exp _{\tilde{\rho}}\left(\mathbb{T}_{z}\left(E_{\infty}\right)\right)} .
$$

Observe that $H\left(\widetilde{\rho} / O_{E}[z]\right)$ is an $A[z]$-module via $\widetilde{\rho}$, and furthermore $H\left(\widetilde{\rho} / O_{E}[z]\right)$ is a finite $\mathbb{F}_{q}[z]$-module ( $[$, Proposition 2$)$. Let's set:

$$
V=\left\{x \in H\left(\widetilde{\rho} / O_{E}[z]\right),(z-1) x=0\right\} .
$$

Since ker ev $=(z-1) \mathbb{T}_{z}\left(E_{\infty}\right)$, the multiplication by $z-1$ on $H\left(\widetilde{\rho} / O_{E}\right)$ gives rise to an exact sequence of finite $A$-modules:

$$
0 \rightarrow V \rightarrow H\left(\widetilde{\rho} / O_{E}[z]\right) \rightarrow H\left(\widetilde{\rho} / O_{E}[z]\right) \rightarrow H\left(\rho / O_{E}\right) \rightarrow 0 .
$$

Thus:

$$
\operatorname{Fitt}_{A} V=\operatorname{Fitt}_{A} H\left(\rho / O_{E}\right) .
$$

Now, let's consider the homomorphism of $\mathbb{F}_{q}[z]$-modules $\alpha: \mathbb{T}_{z}\left(E_{\infty}\right) \rightarrow \mathbb{T}_{z}\left(E_{\infty}\right)$ given by:

Observe that:

$$
\forall x \in \mathbb{T}_{z}\left(E_{\infty}\right), \quad \alpha(x)=\frac{\exp _{\tilde{\rho}}(x)-\exp _{\rho}(x)}{z-1} .
$$

$$
(z-1) \alpha\left(U\left(\rho / O_{E}\right)\right) \subset O_{E}+\exp _{\widetilde{\rho}}\left(\mathbb{T}_{z}\left(E_{\infty}\right)\right),
$$




$$
\forall a \in A, \forall x \in U\left(\rho\left(O_{E}\right)\right), \quad \alpha(a x)-\widetilde{\rho}_{a}(\alpha(x)) \in O_{E}[z] .
$$

Thus $\alpha$ induces a homomorphism of $A$-modules:

$$
\bar{\alpha}: U\left(\rho / O_{E}\right) \rightarrow V .
$$

By [9], Proposition 3, this homomorphism is surjective and its kernel is precisely $U_{S t}\left(\rho / O_{E}\right)$. The theorem follows.

\subsection{Co-volumes.}

Let $V$ be a finite dimensional $K_{\infty}$-vector space of dimension $n \geq 1$. An $A$-lattice in $V$ is a discrete and cocompact sub- $A$-module of $V$.

Lemma 2.8. Let $M, N$ be two A-lattices in $V$. Then there exists an isomorphism of $K_{\infty}$-vector spaces $\sigma: V \rightarrow V$ such that:

$$
\sigma(M) \subset N .
$$

Proof. Since $A$ is a Dedekind domain, there exist two non-zero ideals $I, J$ of $A$, and two $K_{\infty}$-basis $\left\{e_{1}, \ldots e_{n}\right\},\left\{f_{1}, \ldots, f_{n}\right\}$ of $V$, such that:

$$
\begin{aligned}
& M=\oplus_{j=1}^{n-1} A e_{j} \oplus I e_{n}, \\
& N=\oplus_{j=1}^{n-1} A f_{j} \oplus J f_{n} .
\end{aligned}
$$

Furthermore, $M$ and $N$ are isomorphic as $A$-modules if and only if $I$ and $J$ have the same class in the ideal class group $\operatorname{Pic}(A)$ of $A$. Let $x \in I^{-1} J \backslash\{0\}$. Let $\sigma: V \rightarrow V$ such that:

$$
\begin{aligned}
\sigma\left(e_{j}\right) & =f_{j}, \quad j=1, \ldots, n-1, \\
\sigma\left(e_{n}\right) & =x f_{n} .
\end{aligned}
$$

Then:

$$
\sigma(M) \subset N .
$$

Note that if $M$ and $N$ are isomorphic $A$-modules then we can select $x \in K^{\times}$such that $I^{-1} J=x A$ and in this case $\sigma(M)=N$.

Lemma 2.9. Let $M, N$ be two A-lattices in $V$. Let $\sigma_{1}, \sigma_{2}: V \rightarrow V$ be two isomorphisms of $K_{\infty}$-vector spaces such that $\sigma_{i}(M) \subset N, i=1,2$. Then:

$$
\frac{\operatorname{det}_{K_{\infty}} \sigma_{1}}{\operatorname{sgn}\left(\operatorname{det}_{K_{\infty}} \sigma_{1}\right)}\left[\frac{N}{\sigma_{1}(M)}\right]_{A}^{-1}=\frac{\operatorname{det}_{K_{\infty}} \sigma_{2}}{\operatorname{sgn}\left(\operatorname{det}_{K_{\infty}} \sigma_{2}\right)}\left[\frac{N}{\sigma_{2}(M)}\right]_{A}^{-1} .
$$

Proof. Let $\sigma=\sigma_{1} \sigma_{2}^{-1}$. Since $\sigma\left(\sigma_{2}(M)\right)=\sigma_{1}(M) \subset N$, with $\sigma_{2}(M) \subset N$, we can find $a \in A$ with $\operatorname{sgn} a=1$ such that $a \sigma(N) \subset N$. Set $U=\frac{1}{a} \sigma_{2}(M) \cap N$. Then the multiplication by $a$ induces an exact sequence of finite $A$-modules:

$$
0 \longrightarrow \frac{U}{\sigma_{2}(M)} \longrightarrow \frac{N}{\sigma_{2}(M)} \stackrel{a}{\longrightarrow} \frac{N}{\sigma_{2}(M)} \longrightarrow \frac{N}{a N} \longrightarrow 0
$$

from which we deduce

$$
\left[\frac{U}{\sigma_{2}(M)}\right]_{A}=\left[\frac{N}{a N}\right]_{A}=a^{n} .
$$

And $a \sigma$ similarly induces an exact sequence of finite $A$-modules:

$$
0 \longrightarrow \frac{U}{\sigma_{2}(M)} \longrightarrow \frac{N}{\sigma_{2}(M)} \stackrel{a \sigma}{\longrightarrow} \frac{N}{\sigma_{1}(M)} \longrightarrow \frac{N}{a \sigma(N)} \longrightarrow 0 .
$$


We get:

$$
\begin{aligned}
{\left[\frac{N}{\sigma_{1}(M)}\right]_{A} } & =\left[\frac{N}{\sigma_{2}(M)}\right]_{A}\left[\frac{U}{\sigma_{2}(M)}\right]_{A}^{-1}\left[\frac{N}{a \sigma(N)}\right]_{A}=\left[\frac{N}{\sigma_{2}(M)}\right]_{A} a^{-n} \frac{\operatorname{det}_{K_{\infty}}(a \sigma)}{\operatorname{sgn}\left(\operatorname{det}_{K_{\infty}}(a \sigma)\right)} \\
& =\left[\frac{N}{\sigma_{2}(M)}\right]_{A} \frac{\operatorname{det}_{K_{\infty}}(\sigma)}{\operatorname{sgn}\left(\operatorname{det}_{K_{\infty}}(\sigma)\right)} .
\end{aligned}
$$

The lemma follows.

Let $M, N$ be two $A$-lattices in $V$. By Lemma 2.8, there exists an isomorphism of $K_{\infty}$-vector spaces $\sigma: V \rightarrow V$ such that $\sigma(M) \subset N$, we set:

$$
[M: N]_{A}=\frac{\operatorname{det}_{K_{\infty}} \sigma}{\operatorname{sgn}\left(\operatorname{det}_{K_{\infty}} \sigma\right)}\left[\frac{N}{\sigma(M)}\right]_{A}^{-1} .
$$

By Lemma 2.9, this is well-defined. In particular, if $M, N$ are two $A$-lattices in $V$ such that $N \subset M$, then:

$$
[M: N]_{A}=\left[\frac{M}{N}\right]_{A}
$$

If $M, N, U$ are three $A$-lattices in $V$, we get:

$$
[M: N]_{A}=[M: U]_{A}[U: N]_{A} .
$$

Let $F / K$ be a finite extension such that there exists a unique place of $F$ above $\infty$ (still denoted by $\infty$ ). Let $A^{\prime}$ be the integral closure of $A$ in $F$. We assume that $V$ is also an $F_{\infty}$-vector space. Let $[\cdot]_{A^{\prime}}: \mathcal{I}\left(A^{\prime}\right) \rightarrow \bar{K}_{\infty}^{\times}$be the map constructed as in Section 1 with the help of the choice of a uniformizer $\pi^{\prime} \in F_{\infty}^{\times}$. Let $N_{F_{\infty} / K_{\infty}}$ : $F_{\infty} \rightarrow K_{\infty}$ be the usual norm map.

Lemma 2.10. Let $M, N$ be two $A^{\prime}$-lattices in $V$. Then there exists an integer $m \geq 1$ such that $[M: N]_{A^{\prime}}^{m} \in F_{\infty}^{\times},[M: N]_{A}^{m} \in K_{\infty}^{\times}$, and:

$$
N_{F_{\infty} / K_{\infty}}\left([M: N]_{A^{\prime}}^{m}\right)=[M: N]_{A}^{m} .
$$

Proof. Let $\sigma: V \rightarrow V$ be an isomorphism of $F_{\infty}$-vector spaces such that $\sigma(M) \subset N$, and we set: $I^{\prime}=\operatorname{Fitt}_{A^{\prime}} \frac{N}{\sigma(M)}$. Then:

$$
\operatorname{Fitt}_{A} \frac{N}{\sigma(M)}=N_{F / K}\left(I^{\prime}\right)
$$

Let $m \geq 1$ be an integer such that:

$$
I^{\prime m}=x A^{\prime}, \quad x \in A^{\prime} \backslash\{0\} .
$$

Then:

Furthermore, we have:

$$
[M: N]_{A^{\prime}}^{m}=\left(\frac{\operatorname{det}_{F_{\infty}} \sigma}{\operatorname{sgn}^{\prime}\left(\operatorname{det}_{F_{\infty}} \sigma\right)}\right)^{m} \frac{\operatorname{sgn}^{\prime}(x)}{x} .
$$

$$
\operatorname{det}_{K_{\infty}} \sigma=N_{F_{\infty} / K_{\infty}}\left(\operatorname{det}_{F_{\infty}} \sigma\right)
$$

Thus:

Therefore:

$$
[M: N]_{A}^{m}=\left(\frac{N_{F_{\infty} / K_{\infty}}\left(\operatorname{det}_{F_{\infty}} \sigma\right)}{\operatorname{sgn}\left(N_{F_{\infty} / K_{\infty}}\left(\operatorname{det}_{F_{\infty}} \sigma\right)\right)}\right)^{m} \frac{\operatorname{sgn}\left(N_{F / K}(x)\right)}{N_{F / K}(x)} .
$$

The lemma follows.

$$
N_{F_{\infty} / K_{\infty}}\left([M: N]_{A^{\prime}}^{m}\right) \in[M: N]_{A}^{m} \mathbb{F}_{\infty}^{\times}
$$




\subsection{Regulator of Stark units and $L$-series.}

Let $E / K$ be a finite extension, $E \subset \mathbb{C}_{\infty}$. Recall that $E_{\infty}=E \otimes_{K} K_{\infty}$. If $M$ is an $A$-lattice in $E_{\infty}$, then we call $\left[O_{E}: M\right]_{A}$ the A-regulator of $M$.

Definition 2.11. Let $\rho: A \hookrightarrow O_{E}\{\tau\}$ be a Drinfeld module of rank $r \geq 1$. We define the regulator of Stark units associated to $\rho / O_{E}$ by $\left[O_{E}: U_{S t}\left(\rho / O_{E}\right)\right]_{A}$.

Proposition 2.12. Let $\rho: A \hookrightarrow O_{E}\{\tau\}$ be a Drinfeld module of rank $r \geq 1$. We have:

$$
\left[O_{E}: U_{S t}\left(\rho / O_{E}\right)\right]_{A} \in U_{\infty}
$$

Furthermore, the regulator of Stark units relative to $\rho / O_{E}$ depends on $\rho, A$ and $O_{E}$, not on the choice of $\pi$.

Proof. Let $\theta \in A \backslash \mathbb{F}_{q}$, and let $L=\mathbb{F}_{q}(\theta), B=\mathbb{F}_{q}[\theta]$. Let $[\cdot]_{B}: \mathcal{I}(B) \rightarrow L_{\infty}^{\times}$be the map as in Section 1 associated to the choice of $\frac{1}{\theta}$ as a uniformizer of $L_{\infty}$. Then, by Theorem 2.7, we have:

$$
\left[O_{E}: U_{S t}\left(\rho / O_{E}\right)\right]_{B}=\left[O_{E}: U\left(\rho / O_{E}\right)\right]_{B}\left[H\left(\rho / O_{E}\right)\right]_{B} .
$$

Then, by [22], Theorem 2, we get:

$$
\left[O_{E}: U_{S t}\left(\rho / O_{E}\right)\right]_{B} \in 1+\frac{1}{\theta} \mathbb{F}_{q}\left[\left[\frac{1}{\theta}\right]\right] .
$$

Now, by Lemma 2.10, there exists an integer $m \geq 1$ such that:

$$
N_{K_{\infty} / L_{\infty}}\left(\left[O_{E}: U_{S t}\left(\rho / O_{E}\right)\right]_{A}^{m}\right)=\left[O_{E}: U_{S t}\left(\rho / O_{E}\right)\right]_{B}^{m} .
$$

This implies:

$$
v_{\infty}\left(\left[O_{E}: U_{S t}\left(\rho / O_{E}\right)\right]_{A}\right)=0
$$

Thus:

$$
\left[O_{E}: U_{S t}\left(\rho / O_{E}\right)\right]_{A} \in \overline{\mathbb{F}}_{q}^{\times} \times U_{\infty} .
$$

But $\operatorname{sgn}\left(\left[O_{E}: U_{S t}\left(\rho / O_{E}\right)\right]_{A}\right)=1$, thus:

$$
\left[O_{E}: U_{S t}\left(\rho / O_{E}\right)\right]_{A} \in U_{\infty}
$$

Let $\pi^{\prime}$ be another uniformizer of $K_{\infty}$, and let $[\cdot]_{A}^{\prime}: \mathcal{I}(A) \rightarrow \bar{K}_{\infty}^{\times}$be the map as in Section 1 associated to $\pi^{\prime}$. Then, by the above discussion, we get:

$$
\left[O_{E}: U_{S t}\left(\rho / O_{E}\right)\right]_{A}^{\prime} \in U_{\infty} \text {. }
$$

Again, by Lemma 2.10 there exists an integer $m^{\prime} \geq 1$ such that:

$$
\left(\left[O_{E}: U_{S t}\left(\rho / O_{E}\right)\right]_{A}^{\prime}\right)^{m^{\prime}}=\left[O_{E}: U_{S t}\left(\rho / O_{E}\right)\right]_{A}^{m^{\prime}} .
$$

Since $\left[O_{E}: U_{S t}\left(\rho / O_{E}\right)\right]_{A}^{\prime},\left[O_{E}: U_{S t}\left(\rho / O_{E}\right)\right]_{A} \in U_{\infty}$, we get:

$$
\left[O_{E}: U_{S t}\left(\rho / O_{E}\right)\right]_{A}^{\prime}=\left[O_{E}: U_{S t}\left(\rho / O_{E}\right)\right]_{A} .
$$

This concludes the proof of the proposition.

Let's set:

$$
\alpha_{A}\left(\rho / O_{E}\right):=\frac{L_{A}\left(\rho / O_{E}\right)}{\left[O_{E}: U_{S t}\left(\rho / O_{E}\right)\right]_{A}} \in \bar{K}_{\infty}^{\times} .
$$

By Proposition 2.12 and Remark 2.2, $\alpha_{A}\left(\rho / O_{E}\right)$ depends on $A, \rho$, and $O_{E}$, not on the choice of $\pi$. Furthermore:

$$
\alpha_{A}\left(\rho / O_{E}\right) \in U_{\infty} .
$$


Let's also observe that, if $p^{k}$ is the exact power of $p$ dividing $|\operatorname{Pic}(A)|$, then:

$$
\alpha_{A}\left(\rho / O_{E}\right)^{p^{k} d_{\infty}} \in K_{\infty}^{\times} .
$$

We have the fundamental result due to L. Taelman (23], Theorem 1):

Theorem 2.13 (Taelman). Assume that the genus of $K$ is zero and $d_{\infty}=1$. Then:

$$
\alpha_{A}\left(\rho / O_{E}\right)=1 .
$$

Proof. Select $\theta \in A \backslash \mathbb{F}_{q}$ such that $v_{\infty}(\theta)=1$. Then $A=\mathbb{F}_{q}[\theta]$. Let $[\cdot]_{A}: \mathcal{I}(A) \rightarrow K_{\infty}^{\times}$ be the map as in Section 1 associated to the choice of $\frac{1}{\theta}$ as a uniformizer of $K_{\infty}$. Then, by Proposition 2.12, Theorem 2.7 and 23, Theorem 1:

$$
\left[O_{E}: U_{S t}\left(\rho / O_{E}\right)\right]_{A}=\left[O_{E}: U\left(\rho / O_{E}\right)\right]_{A}\left[H\left(\rho / O_{E}\right)\right]_{A}=L_{A}\left(\rho / O_{E}\right) .
$$

This concludes the proof of the theorem.

Corollary 2.14.

1) Let $F / K$ be a finite extension, $F \subset E$, and such that there exists a unique place of $F$ above $\infty$ (still denoted by $\infty)$. Let $A^{\prime}$ be the integral closure of $A$ in $F$. Let $N_{F_{\infty} / K_{\infty}}: F_{\infty} \rightarrow K_{\infty}$ be the usual norm map. Then, there exists an integer $k \geq 1$ such that $\alpha_{A^{\prime}}\left(\rho / O_{E}\right)^{k} \in F_{\infty}^{\times}, \alpha_{A}\left(\rho / O_{E}\right)^{k} \in K_{\infty}^{\times}$, and:

$$
N_{F_{\infty} / K_{\infty}}\left(\alpha_{A^{\prime}}\left(\rho / O_{E}\right)^{k}\right)=\alpha_{A}\left(\rho / O_{E}\right)^{k} .
$$

In particular, $\alpha_{A^{\prime}}\left(\rho / O_{E}\right)=1 \Rightarrow \alpha_{A}\left(\rho / O_{E}\right)=1$.

2) If there exists an integer $m \geq 1$ such that $\alpha_{A}\left(\rho / O_{E}\right)^{m} \in K^{\times}$, then $\alpha_{A}\left(\rho / O_{E}\right)=$

1. In particular, if $\sigma\left(\alpha_{A}\left(\rho / O_{E}\right)\right)=\alpha_{A}\left(\rho^{\sigma} / \sigma\left(O_{E}\right)\right)$ for all $\sigma \in \operatorname{Aut}_{K}\left(\mathbb{C}_{\infty}\right)$, then $\alpha_{A}\left(\rho / O_{E}\right)=1$.

Proof.

1) The first assertion is a consequence of Corollary 2.3 and Lemma 2.10 If $\alpha_{A^{\prime}}\left(\rho / O_{E}\right)=1$, then there exists an integer $k \geq 1$ such that $\alpha_{A}\left(\rho / O_{E}\right)^{k}=1$. But, since $\operatorname{sgn}\left(\alpha_{A}\left(\rho / O_{E}\right)\right)=1$, we get $\alpha_{A}\left(\rho / O_{E}\right)=1$.

2) Let $x=\alpha_{A}\left(\rho / O_{E}\right)^{m} \in K^{\times}$. Let $P$ be a maximal ideal of $A$, and select an integer $l \geq 1$ such that $P^{l}$ is a principal ideal. Let $\theta \in A \backslash \mathbb{F}_{q}$ such that $P^{l}=\theta A$. Let $L=\mathbb{F}_{q}(\theta)$ and $B=\mathbb{F}_{q}[\theta]$. Then, by Taelman's Theorem (Theorem 2.13), we have:

$$
\alpha_{B}\left(\rho / O_{E}\right)=1 \text {. }
$$

Therefore, by 1), we have:

$$
N_{K / L}(x) \in \mathbb{F}_{q}^{\times} .
$$

Since $P$ is the only maximal ideal of $A$ above $\theta B$, we deduce that $x$ is a $P$-adic unit. Since this is true for all maximal ideal of $A$, we get:

$$
x \in \mathbb{F}_{q}^{\times} \text {. }
$$

But, $\operatorname{sgn}\left(\alpha_{A}\left(\rho / O_{E}\right)\right)=1$, thus: $\alpha_{A}\left(\rho / O_{E}\right)=1$.

Let's assume that $\sigma\left(\alpha_{A}\left(\rho / O_{E}\right)\right)=\alpha_{A}\left(\rho^{\sigma} / \sigma\left(O_{E}\right)\right)$ for all $\sigma \in \operatorname{Aut}_{K}\left(\mathbb{C}_{\infty}\right)$. Let $\sigma \in \operatorname{Aut}_{K}\left(\mathbb{C}_{\infty}\right)$. Let $\mathfrak{P}$ be a maximal ideal of $O_{E}$, then:

$$
\begin{gathered}
{\left[\frac{\sigma\left(O_{E}\right)}{\sigma(\mathfrak{P})}\right]_{A}=\left[\frac{O_{E}}{\mathfrak{P}}\right]_{A},} \\
{\left[\rho^{\sigma}\left(\frac{\sigma\left(O_{E}\right)}{\sigma(\mathfrak{P})}\right)\right]_{A}=\left[\rho\left(\frac{O_{E}}{\mathfrak{P}}\right)\right]_{A} .}
\end{gathered}
$$


Thus:

$$
L_{A}\left(\rho^{\sigma} / \sigma\left(O_{E}\right)\right)=L_{A}\left(\rho / O_{E}\right) .
$$

Observe that $\sigma$ induces a $K_{\infty}$-algebra isomorphism:

$$
E_{\infty} \simeq \sigma(E)_{\infty} .
$$

Note that $\exp _{\tilde{\rho}}: E[[z]] \rightarrow E[[z]]$ is an $\mathbb{F}_{q}[[z]]$-algebra isomorphism. Therefore:

$$
U\left(\widetilde{\rho} / O_{E}[z]\right) \subset E[[z]] .
$$

Thus:

$$
U\left(\widetilde{\rho^{\sigma}} / \sigma\left(O_{E}\right)[z]\right)=\sigma\left(U\left(\widetilde{\rho} / O_{E}[z]\right)\right) .
$$

By the definition of Stark units, we get:

$$
U_{S t}\left(\rho^{\sigma} / \sigma\left(O_{E}\right)\right)=\sigma\left(U_{S t}\left(\rho / O_{E}\right)\right) .
$$

Thus:

$$
\left[\sigma\left(O_{E}\right): U_{S t}\left(\rho^{\sigma} / \sigma\left(O_{E}\right)\right)\right]_{A}=\left[O_{E}: U_{S t}\left(\rho / O_{E}\right)\right]_{A} .
$$

Therefore:

$$
\alpha_{A}\left(\rho^{\sigma} / \sigma\left(O_{E}\right)\right)=\alpha_{A}\left(\rho / O_{E}\right) .
$$

We get:

$$
\forall \sigma \in \operatorname{Aut}_{K}\left(\mathbb{C}_{\infty}\right), \quad \sigma\left(\alpha_{A}\left(\rho / O_{E}\right)\right)=\alpha_{A}\left(\rho / O_{E}\right) .
$$

This implies that $\alpha_{A}\left(\rho / O_{E}\right)$ is algebraic over $K$ and that there exists an integer $k \geq 0$ such that:

$$
\alpha_{A}\left(\rho / O_{E}\right)^{p^{k}} \in K^{\times} .
$$

Therefore:

$$
\alpha_{A}\left(\rho / O_{E}\right)=1
$$

We do not know whether $\alpha_{A}\left(\rho / O_{E}\right)$ is algebraic over $K$, and it might be too naive to expect that $\alpha_{A}\left(\rho / O_{E}\right)=1$ in general. However, in the next section, we will prove that, if $\phi$ is a sign-normalized rank one Drinfeld module and $E / K$ is a finite abelian extension such that $H \subset E$, then $\alpha_{A}\left(\phi / O_{E}\right)=1$ (Theorem 3.10 ). L. Taelman informed us that the class formula ([23, Theorem 1$)$ has been generalized by C. Debry to the case where $A$ is a principal ideal domain.

We also prove below that $\alpha_{A}\left(\phi / O_{E}\right)$ is invariant under isogeny, which could be considered as an analogue of the isogeny invariance of the Birch and SwinnertonDyer conjecture due to Tate [24:

Theorem 2.15. Let $E / K$ be a finite extension and let $\rho, \phi: A \rightarrow O_{E}\{\tau\}$ be two Drinfeld A-modules such that there exists $u \in O_{E}\{\tau\} \backslash\{0\}$ with the following property:

$$
\forall a \in A, \quad \rho_{a} u=u \phi_{a}
$$

then:

$$
\alpha_{A}\left(\rho / O_{E}\right)=\alpha_{A}\left(\phi / O_{E}\right) .
$$


Proof. Let $\mathfrak{P}$ be a maximal ideal of $O_{E}$ such that $u \neq \equiv 0(\bmod \mathfrak{P})$. Then by [18, Theorem 3.5 and Theorem 5.1, we get:

$$
\left[\rho\left(\frac{O_{E}}{\mathfrak{P}}\right)\right]_{A}=\left[\phi\left(\frac{O_{E}}{\mathfrak{P}}\right)\right]_{A} .
$$

This implies that there exists an ideal $I \in \mathcal{I}(A)$ such that:

$$
\frac{L_{A}\left(\rho / O_{E}\right)}{L_{A}\left(\phi / O_{E}\right)}=[I] \text {. }
$$

Let $\zeta \in O_{E} \backslash\{0\}$ be the constant coefficient of $u$. Then we have the following equality in $E\{\{\tau\}\}$ :

$$
\exp _{\rho} \zeta=u \exp _{\phi}
$$

Thus:

$$
\exp _{\widetilde{\rho}} \zeta=\widetilde{u} \exp _{\widetilde{\phi}}
$$

where, if $u=\sum_{i=0}^{m} u_{i} \tau^{i}, u_{i} \in O_{E}, \widetilde{u}=\sum_{i=0}^{m} u_{i} z^{i} \tau^{i}$. This implies that:

$$
\zeta U\left(\widetilde{\phi} / O_{E}[z]\right) \subset U\left(\widetilde{\rho} / O_{E}[z]\right) .
$$

Therefore:

$$
\zeta U_{S t}\left(\phi / O_{E}\right) \subset U_{S t}\left(\rho / O_{E}\right)
$$

We get:

and:

$$
\left[O_{E}: \zeta U_{S t}\left(\phi / O_{E}\right)\right]_{A}=\left[O_{E}: U_{S t}\left(\phi / O_{E}\right)\right]_{A}\left[\frac{O_{E}}{\zeta O_{E}}\right]_{A}
$$

$$
\left[O_{E}: \zeta U_{S t}\left(\phi / O_{E}\right)\right]_{A}=\left[O_{E}: U_{S t}\left(\rho / O_{E}\right)\right]_{A}\left[\frac{U_{S t}\left(\rho / O_{E}\right)}{\zeta U_{S t}\left(\phi / O_{E}\right)}\right]_{A}
$$

Therefore, there exists an element $J \in \mathcal{I}(A)$ such that:

$$
\frac{\left[O_{E}: U_{S t}\left(\rho / O_{E}\right)\right]_{A}}{\left[O_{E}: U_{S t}\left(\phi / O_{E}\right)\right]_{A}}=[J] .
$$

Finally, we get:

$$
\frac{\alpha_{A}\left(\rho / O_{E}\right)}{\alpha_{A}\left(\phi / O_{E}\right)}=\left[I J^{-1}\right]
$$

Let $x=\left(\frac{\alpha_{A}\left(\rho / O_{E}\right)}{\alpha_{A}\left(\phi / O_{E}\right)}\right)^{h\left(q^{d} \infty-1\right)} \in K^{\times}$, where $h=|\operatorname{Pic}(A)|$. Then, by Corollary 2.14, and Theorem 2.13] if $\theta \in A \backslash \mathbb{F}_{q}$, there exists an integer $k \geq 1$ such that:

$$
N_{K_{\infty} / \mathbb{F}_{q}\left(\left(\frac{1}{\theta}\right)\right)}\left(x^{k}\right)=1
$$

But, by Proposition 2.12 $x$ is a principal unit in $K_{\infty}$, thus:

$$
N_{K / \mathbb{F}_{q}(\theta)}(x)=1 .
$$

The above equality being valid for any $\theta \in A \backslash \mathbb{F}_{q}$, by the proof of Corollary 2.14 we deduce that:

$$
x=1 .
$$

Since $\operatorname{sgn}\left(\frac{\alpha_{A}\left(\rho / O_{E}\right)}{\alpha_{A}\left(\phi / O_{E}\right)}\right)=1$, we get:

$$
\frac{\alpha_{A}\left(\rho / O_{E}\right)}{\alpha_{A}\left(\phi / O_{E}\right)}=1
$$




\section{Stark units associated to Sign-normalized Rank one Drinfeld MODULES}

\subsection{Zeta functions.}

In this section, we briefly recall the definition of some zeta functions ([19], Chapter 8).

Recall that if $I \in \mathcal{I}(A)$, we have set:

$$
[I]=\langle I\rangle \pi^{-\frac{\operatorname{deg} I}{d_{\infty}}} \in \bar{K}_{\infty}^{\times}
$$

where $v_{\infty}(\langle I\rangle-1)>0$, and:

$$
\forall x \in K^{\times}, \quad\langle x A\rangle=\frac{x}{\operatorname{sgn}(x)} \pi^{-v_{\infty}(x)} .
$$

Let $\mathbb{S}_{\infty}=\mathbb{C}_{\infty}^{\times} \times \mathbb{Z}_{p}$ be the Goss "complex plane". The group action of $\mathbb{S}_{\infty}$ is written additively. Let $I \in \mathcal{I}(A)$ and $s=(x ; y) \in \mathbb{S}_{\infty}$, we set:

$$
I^{s}=\langle I\rangle^{y} x^{\operatorname{deg} I} \in \mathbb{C}_{\infty}^{\times} .
$$

We have a natural injective group homomorphism: $\mathbb{Z} \rightarrow \mathbb{S}_{\infty}, j \mapsto s_{j}=\left(\pi^{-\frac{j}{d_{\infty}}}, j\right)$. Observe that:

$$
\forall j \in \mathbb{Z}, \forall I \in \mathcal{I}(A), \quad I^{s_{j}}=[I]^{j} .
$$

Let $E / K$ be a finite extension, and let $O_{E}$ be the integral closure of $A$ in $E$. Let $\mathfrak{I}$ be a non-zero ideal of $E$. We have:

$$
\forall j \in \mathbb{Z}, \quad N_{E / K}(\mathfrak{I})^{s_{j}}=\left[\frac{O_{E}}{\mathfrak{I}}\right]_{A}^{j} .
$$

Let $s \in \mathbb{S}_{\infty}$, then the following sum converges in $\mathbb{C}_{\infty}([19]$, Theorem 8.9.2):

$$
\zeta_{O_{E}}(s):=\sum_{d \geq 0} \sum_{\substack{\mathfrak{I} \in \mathcal{I}\left(O_{E}\right), \mathfrak{I} \subset O_{E}, \operatorname{deg}\left(N_{E / K}(\mathfrak{I})\right)=d}} N_{E / K}(\mathfrak{I})^{-s} .
$$

The function $\zeta_{O_{E}}: \mathbb{S}_{\infty} \rightarrow \mathbb{C}_{\infty}$ is called the zeta function attached to $O_{E}$ and $[\cdot]_{A}$. Observe that:

$$
\forall j \in \mathbb{Z}, \quad \zeta_{O_{E}}(j):=\zeta_{O_{E}}\left(s_{j}\right)=\sum_{d \geq 0} \sum_{\substack{\mathfrak{I} \in \mathcal{I}(A), \mathfrak{I} \subset O_{E}, \operatorname{deg}\left(N_{E / K}(\mathfrak{I})\right)=d}}\left[\frac{O_{E}}{\mathfrak{I}}\right]_{A}^{-j} .
$$

In particular:

$$
\zeta_{O_{E}}(1)=\prod_{\mathfrak{P}}\left(1-\frac{1}{\left[\frac{O_{E}}{\mathfrak{P}}\right]_{A}}\right)^{-1} \in \bar{K}_{\infty}^{\times},
$$

where $\mathfrak{P}$ runs through the maximal ideals of $O_{E}$.

Lemma 3.1. Let $H_{A}$ be the Hilbert class field of $A$, i.e. $H_{A} / K$ is the maximal unramified abelian extension of $A$ in which $\infty$ splits completely. If $H_{A} \subset E$, then the function $\zeta_{O_{E}}($.$) depends only on O_{E}$ and $\left.\operatorname{sgn}\right|_{K_{\infty}^{\times}}$.

Proof. Let $\mathfrak{P}$ be a maximal ideal of $O_{E}$. Let $A^{\prime}$ be the integral closure of $A$ in $H_{A}$. Let $P^{\prime}=\mathfrak{P} \cap A^{\prime}, P=\mathfrak{P} \cap A$. By class field theory, $P^{\left[\frac{A^{\prime}}{P^{\prime}}: \frac{A}{P}\right]}$ is a principal ideal. Thus:

$$
N_{E / K}(\mathfrak{P})=\theta A,
$$


for some $\theta \in A \backslash \mathbb{F}_{q}$. Let $j \in \mathbb{N}, j \geq 1$. We have:

$$
\left(1-\frac{1}{\left[\frac{O_{E}}{\mathcal{P}^{\mathfrak{S}}}\right]_{A}^{j}}\right)^{-1}=\frac{\frac{\theta^{j}}{\operatorname{sgn}\left(\theta^{j}\right)}}{\frac{\theta^{j}}{\operatorname{sgn}\left(\theta^{j}\right)}-1} .
$$

But, observe that:

$$
\zeta_{O_{E}}(j)=\prod_{\mathfrak{P}}\left(1-\frac{1}{\left[\frac{O_{E}}{\mathfrak{P}^{\mathfrak{P}}}\right]_{A}^{j}}\right)^{-1} \in U_{\infty} \cap K_{\infty}^{\times} .
$$

The lemma is thus a consequence of [19], Theorem 8.7.1.

\subsection{Background on sign-normalized rank one Drinfeld modules.}

Let $\phi: A \rightarrow \bar{K}_{\infty}\{\tau\}$ be a rank one Drinfeld module such that there exists $i(\phi) \in \mathbb{N}$ with the following property:

$$
\forall a \in A \backslash\{0\}, \quad \phi_{a}=a+\cdots+\operatorname{sgn}(a)^{q^{i(\phi)}} \tau^{\operatorname{deg} a} .
$$

Such a Drinfeld module $\phi$ is said to be sign-normalized. By [19, Theorem 7.2.15, there always exist sign-normalized rank one Drinfeld modules.

From now on, we will fix a sign-normalized rank one Drinfeld module $\phi: A \rightarrow \bar{K}_{\infty}\{\tau\}$.

Let $I_{K}$ be the group of idèles of $K$. Let's consider the following subgroup of the idèles of $K$ :

$$
\left.K^{\times} \operatorname{kersgn}\right|_{K_{\infty}^{\times}} \prod_{v \neq \infty} O_{v}^{\times}
$$

where for a place $v$ of $K, O_{v}$ denotes the valuation ring of the $v$-adic completion of $K$. By class field theory, there exists a unique finite abelian extension $H / K$ such that the reciprocity map induces an isomorphism:

$$
\frac{I_{K}}{\left.K^{\times} \operatorname{ker} \operatorname{sgn}\right|_{K_{\infty}^{\times}} \prod_{v \neq \infty} O_{v}^{\times}} \simeq \operatorname{Gal}(H / K) .
$$

The natural surjective homomorphism $I_{K} \rightarrow \mathcal{I}(A)$ induces an isomorphism given by the Artin map $(., H / K)$ :

$$
\frac{\mathcal{I}(A)}{\mathcal{P}_{+}(A)} \simeq \operatorname{Gal}(H / K)
$$

where $\mathcal{P}_{+}(A)=\{x A, x \in K, \operatorname{sgn}(x)=1\}$. Let $H_{A}$ be the Hilbert class field of $A$, i.e. $H_{A} / K$ corresponds to the following subgroup of the idèles of $K$ :

$$
K^{\times} K_{\infty}^{\times} \prod_{v \neq \infty} O_{v}^{\times} .
$$

Then $H / K$ is unramified outside $\infty$, and $H / H_{A}$ is totally ramified at the places of $H_{A}$ above $\infty$. Furthermore:

$$
\operatorname{Gal}\left(H / H_{A}\right) \simeq \frac{\mathbb{F}_{\infty}^{\times}}{\mathbb{F}_{q}^{\times}}
$$

If $w$ is a place of $H$ above $\infty$, then the $w$-adic completion of $H$ is isomorphic to:

$$
K_{\infty}\left(\left((-1)^{d_{\infty}-1} \pi\right)^{\frac{q-1}{q^{d \infty-1}}}\right) .
$$

We denote by $B$ the integral closure of $A$ in $H$ and set $A^{\prime}=B \cap H_{A}$. We observe that $\mathbb{F}_{\infty} \subset A^{\prime}$. 
We denote by $G$ the Galois group $\operatorname{Gal}(H / K)$. For $I \in \mathcal{I}(A)$, we set:

$$
\sigma_{I}=(I, H / K) \in G \text {. }
$$

By [19, Proposition 7.4.2 and Corollary 7.4.9, the subfield of $\mathbb{C}_{\infty}$ generated by $K$ and the coefficients of $\phi_{a}$ is $H$. Furthermore ([19, Lemma 7.4.5):

$$
\forall a \in A, \quad \phi_{a} \in B\{\tau\} .
$$

Let $I$ be a non-zero ideal of $A$, and let's define $\phi_{I}$ to be the unitary element in $H\{\tau\}$ such that:

$$
H\{\tau\} \phi_{I}=\sum_{a \in I} H\{\tau\} \phi_{a}
$$

We have:

$$
\begin{gathered}
\operatorname{ker} \phi_{I}=\bigcap_{a \in I} \operatorname{ker} \phi_{a}, \\
\phi_{I} \in B\{\tau\}, \\
\operatorname{deg}_{\tau} \phi_{I}=\operatorname{deg} I .
\end{gathered}
$$

We write: $\phi_{I}=\phi_{I, 0}+\cdots+\phi_{I, \operatorname{deg} I} \tau^{\operatorname{deg} I}$ with $\phi_{I, \operatorname{deg} I}=1$ and denote by $\psi(I) \epsilon$ $B \backslash\{0\}$ the constant coefficient $\phi_{I, 0}$ of $\phi_{I}$.

Lemma 3.2. The map $\psi$ extends uniquely into a map $\psi: \mathcal{I}(A) \rightarrow H^{\times}$with the following properties:

1) for all $I, J \in \mathcal{I}(A), \psi(I J)=\sigma_{J}(\psi(I)) \psi(J)$,

2) for all $I \in \mathcal{I}(A), I B=\psi(I) B$,

3) for all $x \in K^{\times}, \psi(x A)=\frac{x}{\operatorname{sgn}(x)^{q^{i(\phi)}}}$.

In particular, we have:

$$
\forall x \in K^{\times}, \quad \sigma_{x A}(\psi(I))=\operatorname{sgn}(x)^{q^{i(\phi)}-q^{i(\phi)+\operatorname{deg} I}} \psi(I) .
$$

Proof. Let $I \in \mathcal{I}(A)$, select $a \in A, \operatorname{sgn}(a)=1$, such that $a I \subset A$. Let's set:

$$
\psi(I):=\frac{\psi(a I)}{a} \in H^{\times} .
$$

By [19], Theorem 7.4.8 and Theorem 7.6.2, the map $\psi: \mathcal{I}(A) \rightarrow H^{\times}$is well-defined and satisfies the desired properties.

Note that the map $\psi$ determines $H$ and $H_{A}$ :

Proposition 3.3. We have:

1) $H=K(\psi(I), I \in \mathcal{I}(A))$;

2) $H_{A}=K\left(\psi(I), I \in \mathcal{I}(A), \operatorname{deg} I \equiv 0\left(\bmod d_{\infty}\right)\right)$.

Proof.

1) Let $\sigma \in \operatorname{Gal}\left(H / K(\psi(I), I \in \mathcal{I}(A))\right.$. Let $J \in \mathcal{I}(A)$ such that $\sigma=\sigma_{J}$. Then:

$$
\forall I \in \mathcal{I}(A), \quad \sigma_{I}(\psi(J))=\psi(J) .
$$

Therefore:

$$
\psi(J) \in K^{\times} .
$$

Since $J B=\psi(J) B$ (Lemma 3.2), we get that $J=x A$ for some $x \in K^{\times}$. Thus, for all $I \in \mathcal{I}(A)$, we get:

$$
\operatorname{sgn}(x)^{q^{i(\phi)}-q^{i(\phi)+\operatorname{deg} I}}=1 .
$$

Since $\operatorname{deg}: \mathcal{I}(A) \rightarrow \mathbb{Z}$ is a surjective group homomorphism, this implies that $\operatorname{sgn}(x) \in \mathbb{F}_{q}^{\times}$and thus $J \in \mathcal{P}_{+}(A)$. Therefore $\sigma=1$. 
2) Set $E=K\left(\psi(I), I \in \mathcal{I}(A), \operatorname{deg} I \equiv 0\left(\bmod d_{\infty}\right)\right)$. Observe that:

$$
\operatorname{Gal}\left(H / H_{A}\right)=\left\{\sigma_{x A}, x \in K^{\times}\right\} .
$$

Thus:

$$
K\left(\mathbb{F}_{\infty}\right) \subset E \subset H_{A}
$$

We also have:

$$
\operatorname{Gal}\left(H_{A} / K\left(\mathbb{F}_{\infty}\right)\right)=\left\{\left(I, H_{A} / K\right), I \in \mathcal{I}(A), \operatorname{deg} I \equiv 0 \quad\left(\bmod d_{\infty}\right)\right\} .
$$

Let $\sigma \in \operatorname{Gal}\left(H_{A} / E\right)$. Then, there exists $J \in \mathcal{I}(A), \operatorname{deg} J \equiv 0\left(\bmod d_{\infty}\right)$, such that $\sigma=\left(J, H_{A} / K\right)$. But for all $I \in \mathcal{I}(A), \operatorname{deg} I \equiv 0\left(\bmod d_{\infty}\right)$, we have:

$$
\psi(I J)=\sigma(\psi(I)) \psi(J)=\psi(I) \psi(J),
$$

and therefore:

$$
\left(I, H_{A} / K\right)(\psi(J))=\psi(J) .
$$

This implies:

$$
\psi(J) \in K\left(\mathbb{F}_{\infty}\right)^{\times} .
$$

But:

$$
J A\left[\mathbb{F}_{\infty}\right]=\psi(J) A\left[\mathbb{F}_{\infty}\right] .
$$

Thus $J^{d_{\infty}}$ is a principal ideal. But:

$$
\psi\left(J^{d_{\infty}}\right)=\psi(J)^{d_{\infty}} .
$$

In particular:

$$
\psi(J)^{d_{\infty} \frac{q^{d} \infty-1}{q-1}} \in K^{\times} .
$$

Thus, if $\delta$ is the Frobenius in $\operatorname{Gal}\left(K\left(\mathbb{F}_{\infty}\right) / K\right)$, there exists $\zeta \in \mathbb{F}_{\infty}^{\times}$such that:

$$
\delta(\psi(J))=\zeta \psi(J) .
$$

Observe that:

$$
N_{\mathbb{F}_{\infty} / \mathbb{F}_{q}}(\zeta)=1
$$

Thus:

$$
\zeta=\frac{\mu}{\delta(\mu)}
$$

for some $\mu \in \mathbb{F}_{\infty}^{\times}$. This implies that:

$$
\psi(J) \mu \in K^{\times} .
$$

Therefore $J$ is a principal ideal and thus $\sigma=1$.

We have the following crucial fact:

Proposition 3.4. Let $E / K$ be a finite extension such that $H \subset E$. Then:

$$
L_{A}\left(\phi / O_{E}\right)=\zeta_{O_{E}}(1)
$$

Proof. Let $\mathfrak{P}$ be a maximal ideal of $O_{E}$. Let $m=\left[\frac{O_{E}}{\mathfrak{P}}: \frac{A}{P}\right]$. Then:

$$
N_{E / K}(\mathfrak{P})=P^{m} \text {. }
$$

Since $H \subset O_{E}$, by class field theory, we get:

$$
P^{m}=\theta A, \quad \text { with } \quad \theta \in A, \operatorname{sgn}(\theta)=1 .
$$

Since $\phi$ is a rank one Drinfeld module, it implies that:

$$
\phi_{\theta} \equiv \tau^{m \operatorname{deg} P} \quad(\bmod \mathfrak{P}) .
$$


This implies that:

We get:

$$
\left[\phi\left(\frac{O_{E}}{\mathfrak{P}}\right)\right]_{A}=\theta-1=\left[\frac{O_{E}}{\mathfrak{P}}\right]_{A}-1 .
$$

$$
L_{A}\left(\phi / O_{E}\right)=\prod_{\mathfrak{P}} \frac{\left[\frac{O_{E}}{\mathfrak{P}}\right]_{A}}{\left[\frac{O_{E}}{\mathfrak{P}}\right]_{A}-1}=\prod_{\mathfrak{P}}\left(1-\frac{1}{\left[\frac{O_{E}}{\mathfrak{P}}\right]_{A}}\right)^{-1}=\zeta_{O_{E}}(1) .
$$

\subsection{Equivariant $A$-harmonic series: a detailed example.}

We keep the notation of Section 3.2. Let $z$ be an indeterminate over $K_{\infty}$, and recall that $\mathbb{T}_{z}\left(K_{\infty}\right)$ denotes the Tate algebra in the variable $z$ with coefficients in $K_{\infty}$. Recall that:

$$
\begin{aligned}
H_{\infty} & =H \otimes_{K} K_{\infty}, \\
\mathbb{T}_{z}\left(H_{\infty}\right) & =H \otimes_{K} \mathbb{T}_{z}\left(K_{\infty}\right) .
\end{aligned}
$$

For $n \in \mathbb{Z}$, we set:

$$
Z_{B}(n ; z)=\sum_{d \geq 0} \sum_{\substack{\mathfrak{I} \in \mathcal{I}(B), \mathfrak{I} \subset B, \operatorname{deg}\left(N_{E / K}(\mathfrak{I})\right)=d}}\left[\frac{O_{E}}{\mathfrak{I}}\right]_{A}^{-n} z^{d} .
$$

Then, by [19, Theorem 8.9.2, for all $n \in \mathbb{Z}, Z_{B}(n ;$.$) defines an entire function on$ $\mathbb{C}_{\infty}$, and:

Observe that:

$$
\forall n \in \mathbb{N}, \quad Z_{B}(-n ; z) \in A[z] .
$$

$$
\forall n \in \mathbb{Z}, \quad Z_{B}(n ; z) \in \mathbb{T}_{z}\left(K_{\infty}\right)
$$

and:

$$
\forall n \geq 1, \quad Z_{B}(n ; z)=\prod_{\mathfrak{P}}\left(1-\frac{z^{\operatorname{deg}\left(N_{H / K}(\mathfrak{P})\right)}}{\left[\frac{O_{E}}{\mathfrak{P}}\right]_{A}^{n}}\right)^{-1} \in \mathbb{T}_{z}\left(K_{\infty}\right)^{\times} .
$$

Finally, we note that:

$$
Z_{B}(1 ; 1)=\zeta_{B}(1)
$$

Recall that $G=\operatorname{Gal}(H / K)$. Then $G \simeq \operatorname{Gal}(H(z) / K(z))$ acts on $\mathbb{T}_{z}\left(H_{\infty}\right)$. We denote by $\mathbb{T}_{z}\left(H_{\infty}\right)[G]$ the non-commutative group ring where the commutation rule is given by:

$$
\forall h, h^{\prime} \in \mathbb{T}_{z}\left(H_{\infty}\right), \forall g, g^{\prime} \in G, \quad h g \cdot h^{\prime} g^{\prime}=h g\left(h^{\prime}\right) g g^{\prime} .
$$

Recall that for $I \in \mathcal{I}(A)$, we have set (3.1):

$$
\sigma_{I}=(I, H / K) \in G \text {. }
$$

Lemma 3.5. Let $n \in \mathbb{Z}$. The following infinite sum converges in $\mathbb{T}_{z}\left(H_{\infty}\right)[G]$ :

$$
\mathcal{L}(\phi / B ; n ; z):=\sum_{d \geq 0} \sum_{\substack{I \in \mathcal{I}(A), I \subset A, \operatorname{deg} I=d}} \frac{z^{\operatorname{deg} I}}{\psi(I)^{n}} \sigma_{I} .
$$

Furthermore, for all $n \geq 1$, we have:

$$
\mathcal{L}(\phi / B ; n ; z)=\prod_{P}\left(1-\frac{z^{\operatorname{deg} P}}{\psi(P)^{n}} \sigma_{P}\right)^{-1} \in\left(\mathbb{T}_{z}\left(H_{\infty}\right)[G]\right)^{\times}
$$


and for all $n \leq 0$ :

$$
\mathcal{L}(\phi / B ; n ; z) \in B[z][G] .
$$

Proof. Let $n \geq 1$. First let's observe that for any place $w$ of $H$ above $\infty$ :

$$
\lim _{I \subset A, \operatorname{deg} I \rightarrow+\infty} w(\psi(I))=+\infty .
$$

Let $P$ be a maximal ideal of $A$. Note that:

$$
\forall k \geq 0, \quad \psi\left(P^{k+1}\right)=\sigma_{P}\left(\psi\left(P^{k}\right)\right) \psi(P)=\sigma_{P}^{k}(\psi(P)) \psi\left(P^{k}\right) .
$$

Thus:

$$
\sum_{m \geq 0} \frac{z^{m \operatorname{deg} P}}{\psi\left(P^{m}\right)^{n}} \sigma_{P}^{m} \in \mathbb{T}_{z}\left(H_{\infty}\right)[G]
$$

and we have:

$$
\left(1-\frac{z^{\operatorname{deg} P}}{\psi(P)^{n}} \sigma_{P}\right)\left(\sum_{m \geq 0} \frac{z^{m \operatorname{deg} P}}{\psi\left(P^{m}\right)^{n}} \sigma_{P}^{m}\right)=\left(\sum_{m \geq 0} \frac{z^{m \operatorname{deg} P}}{\psi\left(P^{m}\right)^{n}} \sigma_{P}^{m}\right)\left(1-\frac{z^{\operatorname{deg} P}}{\psi(P)^{n}} \sigma_{P}\right)=1 .
$$

Thus, we have:

$$
\left(1-\frac{z^{\operatorname{deg} P}}{\psi(P)^{n}} \sigma_{P}\right)^{-1}:=\sum_{m \geq 0} \frac{z^{m \operatorname{deg} P}}{\psi\left(P^{m}\right)^{n}} \sigma_{P}^{m} \in\left(\mathbb{T}_{z}\left(H_{\infty}\right)[G]\right)^{\times} .
$$

Let $P, Q$ be two distinct maximal ideals of $A$. We have:

$\left(1-\frac{z^{\operatorname{deg} P}}{\psi(P)^{n}} \sigma_{P}\right)\left(1-\frac{z^{\operatorname{deg} Q}}{\psi(Q)^{n}} \sigma_{Q}\right)=\left(1-\frac{z^{\operatorname{deg} Q}}{\psi(Q)^{n}} \sigma_{Q}\right)\left(1-\frac{z^{\operatorname{deg} P}}{\psi(P)^{n}} \sigma_{P}\right)=\left(1-\frac{z^{\operatorname{deg}(P Q)}}{\psi(P Q)^{n}} \sigma_{P Q}\right)$.

Therefore:

$$
\mathcal{L}(\phi / B ; n ; z)=\prod_{P}\left(1-\frac{z^{\operatorname{deg} P}}{\psi(P)^{n}} \sigma_{P}\right)^{-1}=\sum_{I \in \mathcal{I}(A), I \subset A} \frac{z^{\operatorname{deg} I}}{\psi(I)^{n}} \sigma_{I} \in\left(\mathbb{T}_{z}\left(H_{\infty}\right)[G]\right)^{\times} .
$$

Let $n \in \mathbb{Z}$. For $d \in \mathbb{N}$, we set:

$$
S_{\psi, d}(B ; n)=\sum_{\substack{I \in \mathcal{I}(A), I \subset A, \operatorname{deg} I=d}} \psi(I)^{-n} \sigma_{I} \in H[G] .
$$

Let $h$ be the order of $\frac{\mathcal{I}(A)}{\mathcal{P}_{+}(A)}$. Let $I_{1}, \ldots, I_{h} \in \mathcal{I}(A) \cap A$ be a system of representatives of $\frac{\mathcal{I}(A)}{\mathcal{P}_{+}(A)}$. Then:

$$
S_{\psi, d}(B ; n)=\sum_{j=1}^{h} \psi\left(I_{j}\right)^{-n} \sigma_{I_{j}} \sum_{\substack{a \in K^{\times}, \operatorname{sgn}(a)=1, a I_{j} \subset A, \operatorname{deg}\left(a I_{j}\right)=d}} a^{-n} .
$$

Now, let's assume that $n \leq 0$. Then, by [5], Lemma 3.2, there exists an integer $d_{0}(n, \psi, H) \in \mathbb{N}$ such that, for all $d \geq d_{0}(n, \psi, H)$, for all $j \in\{1, \ldots, h\}$, we have:

$$
\sum_{\substack{a \in K^{\times}, \operatorname{sgn}(a)=1, a I_{j} \subset A, \operatorname{deg}\left(a I_{j}\right)=d}} a^{-n}=0 .
$$

Therefore, for $d \geq d_{0}(n, \psi, H)$, we have:

$$
S_{\psi, d}(B ; n)=0 .
$$


Thus:

$$
\forall n \in \mathbb{N}, \quad \mathcal{L}(\phi / B ;-n ; z) \in B[z][G] .
$$

The element $\mathcal{L}(\phi / B):=\mathcal{L}(\phi / B ; 1 ; 1) \in\left(H_{\infty}[G]\right)^{\times}$will be called the equivariant $A$-harmonic series attached to $\phi / B$.

Note that $\mathcal{L}(\phi / B ; 1 ; z)$ induces a $\mathbb{T}_{z}\left(K_{\infty}\right)$-linear map $\mathcal{L}(\phi / B ; 1 ; z): \mathbb{T}_{z}\left(H_{\infty}\right) \rightarrow$ $\mathbb{T}_{z}\left(H_{\infty}\right)$. Since $\mathbb{T}_{z}\left(H_{\infty}\right)$ is a free $\mathbb{T}_{z}\left(K_{\infty}\right)$-module of rank $[H: K]$ (recall that $\mathbb{T}_{z}\left(K_{\infty}\right)$ is a principal ideal domain), $\operatorname{det}_{\mathbb{T}_{z}\left(K_{\infty}\right)} \mathcal{L}(\phi / B ; 1 ; z)$ is well-defined. We also observe that $\mathcal{L}(\phi / B)$ induces a $K_{\infty}$-linear map $\mathcal{L}(\phi / B): H_{\infty} \rightarrow H_{\infty}$, and we denote by $\operatorname{det}_{K_{\infty}} \mathcal{L}(\phi / B)$ its determinant. Recall that ev : $\mathbb{T}_{z}\left(H_{\infty}\right) \rightarrow H_{\infty}$ is the $H_{\infty}$-linear map given by:

$$
\forall f \in \mathbb{T}_{z}\left(H_{\infty}\right), \quad \operatorname{ev}(f)=\left.f\right|_{z=1} .
$$

Observe that, if $\left\{e_{1}, \ldots, e_{n}\right\}$ is a $K$-basis of $H / K$ (recall that $n=[H: K]$ ), then:

$$
\begin{aligned}
H_{\infty} & =\oplus_{i=1}^{n} K_{\infty} e_{i}, \\
\mathbb{T}_{z}\left(H_{\infty}\right) & =\oplus_{i=1}^{n} \mathbb{T}_{z}\left(K_{\infty}\right) e_{i} .
\end{aligned}
$$

We deduce that:

$$
\operatorname{det}_{K_{\infty}} \mathcal{L}(\phi / B)=\operatorname{ev}\left(\operatorname{det}_{\mathbb{T}_{z}\left(K_{\infty}\right)} \mathcal{L}(\phi / B ; 1 ; z)\right) .
$$

Theorem 3.6. We have:

$$
\operatorname{det}_{\mathbb{T}_{z}\left(K_{\infty}\right)} \mathcal{L}(\phi / B ; 1 ; z)=Z_{B}(1 ; z) .
$$

In particular:

$$
\operatorname{det}_{K_{\infty}} \mathcal{L}(\phi / B)=\zeta_{B}(1) .
$$

Proof. First, we recall that, by Lemma 3.5 we have the following equality in $\mathbb{T}_{z}\left(H_{\infty}\right)[G]$ :

$$
\prod_{P}\left(1-\frac{z^{\operatorname{deg} P}}{\psi(P)} \sigma_{P}\right)^{-1}=\mathcal{L}(\phi / B ; 1 ; z),
$$

where $P$ runs through the maximal ideals of $A$, and:

$$
\left(1-\frac{z^{\operatorname{deg} P}}{\psi(P)} \sigma_{P}\right)^{-1}=\sum_{n \geq 0} \frac{z^{n \operatorname{deg} P}}{\psi\left(P^{n}\right)} \sigma_{P^{n}} .
$$

By the properties of $\psi$ (Lemma 3.2), we have:

$$
\lim _{N \rightarrow+\infty} \prod_{\operatorname{deg} P \geq N}\left(1-\frac{z^{\operatorname{deg} P}}{\psi(P)} \sigma_{P}\right)^{-1}=1 .
$$

Thus:

$$
\operatorname{det}_{\mathbb{T}_{z}\left(K_{\infty}\right)} \mathcal{L}(\phi / B ; 1 ; z)=\prod_{P} \operatorname{det}_{\mathbb{T}_{z}\left(K_{\infty}\right)}\left(1-\frac{z^{\operatorname{deg} P}}{\psi(P)} \sigma_{P}\right)^{-1} .
$$

Thus, we are led to compute:

$$
\operatorname{det}_{\mathbb{T}_{z}\left(K_{\infty}\right)}\left(1-\frac{z^{\operatorname{deg} P}}{\psi(P)} \sigma_{P}\right) .
$$

But $1-\frac{z^{\operatorname{deg} P}}{\psi(P)} \sigma_{P}$ induces a $K[z]$-linear map on $H[z]$. Thus: 


$$
\operatorname{det}_{\mathbb{T}_{z}\left(K_{\infty}\right)}\left(1-\frac{z^{\operatorname{deg} P}}{\psi(P)} \sigma_{P}\right)=\left.\operatorname{det}_{K[z]}\left(1-\frac{z^{\operatorname{deg} P}}{\psi(P)} \sigma_{P}\right)\right|_{H[z]} .
$$

Let $e \geq 1$ be the order of $P$ in $\frac{\mathcal{I}(A)}{\mathcal{P}_{+}(A)}$. Write $\xi=\left.\frac{z^{\operatorname{deg} P}}{\psi(P)} \sigma_{P}\right|_{H[z]}$. We have $\xi^{e}=$ $\frac{z^{e \operatorname{deg} P}}{\psi\left(P^{e}\right)} \in K[z]$. Since $e$ is the order of $\sigma_{P}$ in $G$, by Dedekind's Theorem $\sigma_{P}^{0}, \sigma_{P}$, $\ldots, \sigma_{P}^{e-1}$ are linearly independent over $H(z)$. We deduce that $X^{e}-\frac{z^{e \operatorname{deg} P}}{\psi\left(P^{e}\right)}$ is the minimal polynomial of $\xi$ over $K(z)$ and also over $H^{\left\langle\sigma_{P}\right\rangle}(z)$, and that:

$$
\left.\operatorname{det}_{K[z]}\left(1-\frac{z^{\operatorname{deg} P}}{\psi(P)} \sigma_{P}\right)\right|_{H[z]}=\left(1-\frac{z^{e \operatorname{deg} P}}{\psi\left(P^{e}\right)}\right)^{\frac{[H: K]}{e}} .
$$

Now, let $\mathfrak{P}$ be a maximal ideal of $B$ above $P$. Then, by class field theory, we have:

$$
\left[\frac{B}{\mathfrak{P}}: \frac{A}{P}\right]=e .
$$

Therefore:

$$
\left[\frac{B}{\mathfrak{P}}\right]_{A}=\psi\left(P^{e}\right)
$$

Thus:

$$
\left.\operatorname{det}_{K[z]}\left(1-\frac{z^{\operatorname{deg} P}}{\psi(P)} \sigma_{P}\right)\right|_{H[z]}=\prod_{\mathfrak{P} \mid P}\left(1-\frac{z^{\operatorname{deg}\left(N_{H / K}(\mathfrak{P})\right)}}{\left[\frac{B}{\mathfrak{P}}\right]_{A}}\right) .
$$

Finally, we get:

$$
\operatorname{det}_{\mathbb{T}_{z}\left(K_{\infty}\right)} \mathcal{L}(\phi / B ; 1 ; z)=\prod_{\mathfrak{P}}\left(1-\frac{z^{\operatorname{deg}\left(N_{H / K}(\mathfrak{P})\right)}}{\left[\frac{B}{\mathfrak{P}}\right]_{A}}\right)^{-1},
$$

where $\mathfrak{P}$ runs through the maximal ideals of $B$. Thus:

$$
\operatorname{det}_{\mathbb{T}_{z}\left(K_{\infty}\right)} \mathcal{L}(\phi / B ; 1 ; z)=Z_{B}(1 ; z) .
$$

Now:

$$
\operatorname{det}_{K_{\infty}} \mathcal{L}(\phi / B)=\operatorname{ev}\left(\operatorname{det}_{T_{z}\left(K_{\infty}\right)} \mathcal{L}(\phi / B ; 1 ; z)\right)=\operatorname{ev}\left(Z_{B}(1 ; z)\right)=\zeta_{B}(1) .
$$

Although this is not evident, the above theorem reflects a class formula à la Taelman which will be proved in Section 3.5.

\subsection{Stark units.}

We keep the notation of the previous sections. We will need the following basic result:

Lemma 3.7. Let $L / K$ be a finite extension, and let $O_{L}$ be the integral closure of $A$ in L. Let $\rho: A \rightarrow O_{L}\{\tau\}$ be a Drinfeld module of rank $r \geq 1$. Let $\exp _{\rho}, \log _{\rho} \in$ $1+L\{\{\tau\}\} \tau$ be such that:

Write:

$$
\begin{gathered}
\forall a \in A, \quad \exp _{\rho} a=\rho_{a} \exp _{\rho} \\
\exp _{\rho} \log _{\rho}=\log _{\rho} \exp _{\rho}=1
\end{gathered}
$$

$$
\begin{aligned}
\exp _{\rho} & =\sum_{i \geq 0} e_{i}(\rho) \tau^{i}, \\
\log _{\rho} & =\sum_{i \geq 0} l_{i}(\rho) \tau^{i},
\end{aligned}
$$


with $e_{i}(\rho), l_{i}(\rho) \in L$.

1) Let $P$ be a maximal ideal of $A$. Let $A_{P}$ be the $P$-adic completion of $A$. Then:

$$
\begin{aligned}
& \forall n \geq 0, \quad P^{q^{n}} e_{n}(\rho) O_{L} \subset P O_{L} \otimes_{A} A_{P}, \\
& \forall n \geq 0, \quad P^{\left[\frac{n}{\operatorname{deg} P}\right]} l_{n}(\rho) O_{L} \subset O_{L} \otimes_{A} A_{P} .
\end{aligned}
$$

2) Let $\sigma: L \hookrightarrow \bar{K}_{\infty}$ be a field homomorphism such that $\left.\sigma\right|_{K}=\operatorname{Id}_{K}$. Then, there exist $n(\rho, \sigma) \in \mathbb{N}, C(\rho, \sigma) \in] 0 ;+\infty[$, such that:

$$
\forall n \geq n(\rho, \sigma), \quad v_{\infty}\left(\sigma\left(e_{n}(\rho)\right)\right) \geq C(\rho, \sigma) n q^{n} .
$$

Proof.

1) Let $\theta \in A \backslash \mathbb{F}_{q}$ such that $\theta A_{P}=P A_{P}$. Let $d=r \operatorname{deg}(\theta)$, and let's write:

$$
\rho_{\theta}=\sum_{j=0}^{d} \rho_{\theta, j} \tau^{j}
$$

From $\exp _{\rho} \theta=\rho_{\theta} \exp _{\rho}$, we get:

$$
\forall n \geq 0, \quad\left(\theta^{q^{n}}-\theta\right) e_{n}(\rho)=\sum_{l=1}^{d} \rho_{\theta, l} e_{n-l}(\rho)^{q^{l}}
$$

where $e_{i}=0$ if $i<0$. Since $e_{0}(\rho)=1$, one proves by induction on $n \geq 0$ that

$$
e_{n}(\rho) \theta^{q^{n}} \in \theta^{\inf \left\{q-1, q^{n}\right\}} O_{L} \otimes_{A} A_{P} .
$$

Observe that:

$$
\forall a \in A, \quad a \log _{\rho}=\log _{\rho} \rho_{a}
$$

Thus:

$$
\forall a \in A, \forall n \geq 0, \quad\left(a-a^{q^{n}}\right) l_{n}(\rho)=\sum_{l=1}^{r \operatorname{deg} a} l_{n-l}(\rho) \rho_{a, l}^{q^{n-l}} .
$$

Thus, if $n \not \equiv 0(\bmod \operatorname{deg} P)$, we get:

$$
l_{n}(\rho) O_{E} \otimes_{A} A_{P} \subset \sum_{l=1}^{n} l_{n-l}(\rho) O_{L} \otimes_{A} A_{P} .
$$

If $n \equiv 0(\bmod \operatorname{deg} P)$, we have:

$$
\left(\theta-\theta^{q^{n}}\right) l_{n}(\rho)=\sum_{l=1}^{d} l_{n-l}(\rho) \rho_{\theta, l}^{q^{n-l}}
$$

In any case, we get:

$$
\theta^{\left[\frac{n}{\operatorname{deg} P}\right]} l_{n}(\rho) \in \sum_{l=1}^{n} \theta^{\left[\frac{n-l}{\operatorname{deg} P}\right]} l_{n-l}(\rho) O_{L} \otimes_{A} A_{P} .
$$

Since $l_{0}(\rho)=1$, we get the desired second assertion by induction on $n \geq 0$.

2) This is a consequence of the proof of [19, Theorem 4.6.9. We give a proof for the convenience of the reader. We keep the previous notation. In particular, let $\theta \in A \backslash \mathbb{F}_{q}$, and write:

$$
\rho_{\theta}=\sum_{j=0}^{r \operatorname{deg}(\theta)} \rho_{\theta, j} \tau^{j}, \quad \text { with } \quad \rho_{\theta, j} \in \bar{K}_{\infty} .
$$


Recall that $\rho_{\theta, 0}=\theta$. Set $d=r \operatorname{deg}(\theta)$. Then:

$$
\forall n \geq 0, \quad\left(\theta^{q^{n}}-\theta\right) e_{n}(\rho)=\sum_{l=1}^{d} \rho_{\theta, l} e_{n-l}(\rho)^{q^{l}} .
$$

Set $u=\frac{\operatorname{deg}(\theta)}{d_{\infty}}=-v_{\infty}(\theta) \geq 1$. We get :

$$
\frac{v_{\infty}\left(e_{n}(\rho)\right)}{q^{n}} \geq u+\inf \left\{\frac{v_{\infty}\left(e_{n-j}(\rho)\right)}{q^{n-j}}+\frac{v_{\infty}\left(\rho_{\theta, j}\right)}{q^{n}}, j=1, \ldots, d\right\} .
$$

Let $\beta \in] 0 ; u\left[\right.$. There exists an integer $n_{0}$ such that:

$$
\forall n \geq n_{0}, \quad \inf \left\{\frac{v_{\infty}\left(\rho_{\theta, j}\right)}{q^{n}}, j=1, \ldots, d\right\} \geq \beta-u
$$

Therefore:

$$
\forall n \geq n_{0}, \quad \frac{v_{\infty}\left(e_{n}(\rho)\right)}{q^{n}} \geq \beta+\inf \left\{\frac{v_{\infty}\left(e_{n-j}(\rho)\right)}{q^{n-j}}, j=1, \ldots, d\right\} .
$$

Thus, for $n \in\left[n_{0} ; n_{0}+d-1\right]$, we get:

$$
\frac{v_{\infty}\left(e_{n}(\rho)\right)}{q^{n}} \geq \beta+\inf \left\{\frac{v_{\infty}\left(e_{n_{0}-j}(\rho)\right)}{q^{n_{0}-j}}, j=1, \ldots, d\right\} .
$$

Set:

$$
C=\inf \left\{\frac{v_{\infty}\left(e_{n_{0}-j}(\rho)\right)}{q^{n_{0}-j}}, j=1, \ldots, d\right\} .
$$

By induction, we show that if $n \geq n_{0}+m d, m \in \mathbb{N}$, then:

$$
\frac{v_{\infty}\left(e_{n}(\rho)\right)}{q^{n}} \geq \beta(m+1)+C .
$$

Therefore there exist $n_{1} \geq n_{0}, C^{\prime}, C \in \mathbb{Q}$, with $C^{\prime}>0$, such that:

$$
\forall n \geq n_{1}, \quad v_{\infty}\left(e_{n}(\rho)\right) \geq C^{\prime} n q^{n}+C .
$$

Let $E / K$ be a finite abelian extension $H \subset E$. Let $G=\operatorname{Gal}(E / K)$. We denote by $S_{E}$ the set of maximal ideals $P$ of $A$ which are wildly ramified in $E / K$ (note that we can have $S_{E}=\emptyset$ ). Let $P$ be a maximal ideal of $A$ such that $P \notin S_{E}$. We fix a maximal ideal $\mathfrak{P}$ of $O_{E}$ above $P$. Let $D_{P} \subset G$ be the decomposition group associated to $P$, i.e. $D_{P}=\{g \in G, g(\mathfrak{P})=\mathfrak{P}\}$. We have a natural surjective homomorphism $D_{P} \rightarrow \operatorname{Gal}\left(\frac{O_{E}}{\mathfrak{P}} / \frac{A}{P}\right), g \mapsto \bar{g}$. Let $I_{P}$ be the inertia group at $P$, i.e. $I_{P}=\operatorname{ker}\left(D_{P} \rightarrow \operatorname{Gal}\left(\frac{O_{E}}{\mathfrak{P}} / \frac{A}{P}\right)\right)$. Then, since $P \notin S_{E}$, we have:

$$
\left|I_{P}\right| \not \equiv 0 \quad(\bmod p) \text {. }
$$

Let $\operatorname{Frob}_{P} \in \operatorname{Gal}\left(\frac{O_{E}}{\mathfrak{P}^{3}} / \frac{A}{P}\right)$ be the Frobenius at $P$, i.e.

We set:

$$
\forall x \in \frac{O_{E}}{\mathfrak{P}}, \quad \operatorname{Frob}_{P}(x)=x^{q^{\operatorname{deg} P}} .
$$

If $P \in S_{E}$, we set:

$$
\sigma_{P, O_{E}}:=\frac{1}{\left|I_{P}\right|} \sum_{g \in D_{P}, \bar{g}=\operatorname{Frob}_{P}} g \in \mathbb{F}_{p}[G]
$$

$$
\sigma_{P, O_{E}}=0 .
$$


Note that, if $L / K$ is a finite abelian extension, $L \subset E$, and if $P$ is unramified in $L$ with $P \notin S_{E}$, then:

$$
\left.\sigma_{P, O_{E}}\right|_{L}=(P, L / K)
$$

If $I \in \mathcal{I}(A), I \subset A, I=\prod_{P} P^{m_{P}}$, we set:

$$
\sigma_{I, O_{E}}=\prod_{P} \sigma_{P, O_{E}}^{m_{P}} \in \mathbb{F}_{p}[G] .
$$

For all $n \in \mathbb{Z}$, we set:

$$
\mathcal{L}\left(\phi / O_{E} ; n ; z\right)=\sum_{d \geq 0} \sum_{\substack{I \in \mathcal{I}(A), I \subset A, \operatorname{deg} I=d}} \frac{z^{d}}{\psi(I)^{n}} \sigma_{I, O_{E}} \in H[G][[z]] .
$$

By the proof of Lemma 3.5, we have:

$$
\forall n \in \mathbb{Z}, \quad \mathcal{L}\left(\phi / O_{E} ; n ; z\right) \in \mathbb{T}_{z}\left(H_{\infty}\right)[G]
$$

and:

$$
\mathcal{L}\left(\phi / O_{E} ; 1 ; z\right)=\prod_{P}\left(1-\frac{z^{\operatorname{deg} P}}{\psi(P)} \sigma_{P, O_{E}}\right)^{-1} \in\left(\mathbb{T}_{z}\left(H_{\infty}\right)[G]\right)^{\times} .
$$

Note that, if $L / K$ is a finite abelian extension, $H \subset L \subset E$, we have:

$$
\left.\mathcal{L}\left(\phi / O_{E} ; 1 ; z\right)\right|_{\mathbb{T}_{z}\left(L_{\infty}\right)}=\left.\left(\prod_{P \in S_{E} \backslash S_{L}}\left(1-\frac{z^{\operatorname{deg} P}}{\psi(P)} \sigma_{P, O_{L}}\right) \mathcal{L}\left(\phi / O_{L} ; 1 ; z\right)\right)\right|_{\mathbb{T}_{z}\left(L_{\infty}\right)} .
$$

We set:

$$
I\left(O_{E}\right)=\prod_{P \in S_{E}} P
$$

Recall that

$$
U\left(\widetilde{\phi} / O_{E}[z]\right)=\left\{f \in \mathbb{T}_{z}\left(E_{\infty}\right), \exp _{\tilde{\phi}}(f) \in O_{E}[z]\right\}
$$

Theorem 3.8. We always have:

$$
\psi\left(I\left(O_{E}\right)\right) \mathcal{L}\left(\phi / O_{E} ; 1 ; z\right) O_{E}[z] \subset U\left(\widetilde{\phi} / O_{E}[z]\right) .
$$

Furthermore, if $S_{E}=\emptyset$, we have an equality:

$$
\mathcal{L}\left(\phi / O_{E} ; 1 ; z\right) O_{E}[z]=U\left(\widetilde{\phi} / O_{E}[z]\right) .
$$

Proof. We divide the proof into several steps.

1) We will first work in $E[[z]]$. Observe that $\exp _{\tilde{\phi}}: E[[z]] \rightarrow \widetilde{\phi}(E[[z]])$ is an isomorphism of $A$-modules. In fact, if we write: $\log _{\phi}=\sum_{i \geq 0} l_{i}(\phi) \tau^{i}$, then we set:

$$
\log _{\widetilde{\phi}}=\sum_{i \geq 0} l_{i}(\phi) z^{i} \tau^{i}
$$

Thus, $\log _{\widetilde{\phi}}$ converges on $E[[z]]$, and $\log _{\widetilde{\phi}} \exp _{\widetilde{\phi}}=\exp _{\widetilde{\phi}} \log _{\widetilde{\phi}}=1$.

2) Let $P$ be a maximal ideal of $A$. Let $R_{P}=S^{-1} O_{E} \subset E$, where $S=A \backslash P$. Then:

$$
P R_{P}=\psi(P) R_{P} .
$$

By Lemma 3.7, we have:

$$
\begin{aligned}
& \exp _{\widetilde{\phi}}\left(P R_{P}[[z]]\right) \subset P R_{P}[[z]], \\
& \log _{\tilde{\phi}}\left(P R_{P}[[z]]\right) \subset P R_{P}[[z]] .
\end{aligned}
$$


Thus:

$$
\exp _{\widetilde{\phi}}\left(P R_{P}[[z]]\right)=P R_{P}[[z]] .
$$

3) Recall that there exists a sign-normalized rank one Drinfeld module $\varphi:=P * \phi$ : $A \hookrightarrow B\{\tau\}$ such that:

$$
\forall a \in A, \quad \phi_{P} \phi_{a}=\varphi_{a} \phi_{P} .
$$

Furthermore ([19], Theorem 7.4.8):

$$
\forall a \in A, \quad \varphi_{a}=\sigma_{P}\left(\phi_{a}\right):=\sum_{i=0}^{r \operatorname{deg} a} \sigma_{P}\left(\phi_{a, i}\right) \tau^{i} .
$$

Thus:

$$
\begin{aligned}
\exp _{\varphi}=\sigma_{P}\left(\exp _{\phi}\right) & :=\sum_{i \geq 0} \sigma_{P}\left(e_{i}(\phi)\right) \tau^{i} \\
\log _{\varphi}=\sigma_{P}\left(\log _{\phi}\right) & :=\sum_{i \geq 0} \sigma_{P}\left(l_{i}(\phi)\right) \tau^{i}
\end{aligned}
$$

In particular:

$$
\begin{gathered}
\phi_{P} \exp _{\phi}=\sigma_{P}\left(\exp _{\phi}\right) \psi(P), \\
\psi(P) \log _{\phi}=\sigma_{P}\left(\log _{\phi}\right) \phi_{P} .
\end{gathered}
$$

The same properties hold for $\widetilde{\phi}$.

4) Let's set:

$$
U\left(\widetilde{\phi} / R_{P}[[z]]\right)=\left\{x \in E[[z]] ; \exp _{\widetilde{\phi}}(x) \in R_{P}[[z]]\right\} .
$$

Let's assume that $P \notin S_{E}$. Then, by 1) and 2), $\exp _{\tilde{\phi}}$ induces an isomorphism of $A$-modules:

$$
\frac{E[[z]]}{P R_{P}[[z]]} \simeq \widetilde{\phi}\left(\frac{E[[z]]}{P R_{P}[[z]]}\right) .
$$

Therefore, we get an isomorphism of $A$-modules:

$$
\frac{U\left(\widetilde{\phi} / R_{P}[[z]]\right)}{P R_{P}[[z]]} \simeq \widetilde{\phi}\left(\frac{R_{P}[[z]]}{P R_{P}[[z]]}\right)
$$

Now observe that:

$$
\left(\widetilde{\phi}_{P}-z^{\operatorname{deg} P} \sigma_{P, O_{E}}\right) \widetilde{\phi}\left(\frac{R_{P}[[z]]}{P R_{P}[[z]]}\right)=\{0\} .
$$

Furthermore, if $x \in E[[z]] \backslash R_{P}[[z]]$, then one can easily verify that:

$$
\left(\widetilde{\phi}_{P}-z^{\operatorname{deg} P} \sigma_{P, O_{E}}\right)(x) \notin P R_{P}[[z]] .
$$

Thus:

$$
\widetilde{\phi}\left(\frac{R_{P}[[z]]}{P R_{P}[[z]]}\right)=\left\{x \in \widetilde{\phi}\left(\frac{E[[z]]}{P R_{P}[[z]]}\right),\left(\widetilde{\phi}_{P}-z^{\operatorname{deg} P} \sigma_{P, O_{E}}\right)(x)=0\right\} .
$$

Let $x \in E[[z]]$, we deduce that:

$$
x \in U\left(\widetilde{\phi} / R_{P}[[z]]\right) \Leftrightarrow\left(\widetilde{\phi}_{P}-z^{\operatorname{deg} P} \sigma_{P, O_{E}}\right)\left(\exp _{\widetilde{\phi}}(x)\right) \in P R_{P}[[z]] .
$$

Observe that, by 3 ), we have:

$$
\sigma_{P, O_{E}}\left(\exp _{\widetilde{\phi}}\right)=\exp _{\widetilde{\varphi}},
$$

and also:

$$
\widetilde{\phi}_{P} \exp _{\tilde{\phi}}=\sigma_{P, O_{E}}\left(\exp _{\tilde{\phi}}\right) \psi(P) .
$$


Thus:

$$
x \in U\left(\widetilde{\phi} / R_{P}[[z]]\right) \Leftrightarrow \exp _{\widetilde{\varphi}}\left(\psi(P) x-z^{\operatorname{deg} P} \sigma_{P, O_{E}}(x)\right) \in P R_{P}[[z]] .
$$

Applying (3.2) for $\varphi$, we have:

$$
x \in U\left(\widetilde{\phi} / R_{P}[[z]]\right) \Leftrightarrow \psi(P) x-z^{\operatorname{deg} P} \sigma_{P, O_{E}}(x) \in P R_{P}[[z]] .
$$

Thus:

$$
U\left(\widetilde{\phi} / R_{P}[[z]]\right)=\left(1-\frac{z^{\operatorname{deg} P}}{\psi(P)} \sigma_{P, O_{E}}\right)^{-1} R_{P}[[z]]
$$

5) Let $P$ be a maximal ideal of $A$. If $P \notin S_{E}$, by 4 ), we have:

$$
U\left(\widetilde{\phi} / R_{P}[[z]]\right)=\psi\left(I\left(O_{E}\right)\right) \mathcal{L}\left(\phi / O_{E} ; 1 ; z\right) R_{P}[[z]]=\left(1-\frac{z^{\operatorname{deg} P}}{\psi(P)} \sigma_{P, O_{E}}\right)^{-1} R_{P}[[z]] .
$$

If $P \in S_{E}$, then:

$$
\psi\left(I\left(O_{E}\right)\right) \mathcal{L}\left(\phi / O_{E} ; 1 ; z\right) R_{P}[[z]]=P R_{P}[[z]] \subset U\left(\widetilde{\phi} / R_{P}[[z]]\right) .
$$

Since $\psi\left(I\left(O_{E}\right)\right) \mathcal{L}\left(\phi / O_{E} ; 1 ; z\right) \in \mathbb{T}_{z}\left(H_{\infty}\right)[G]$, we get:

$$
\psi\left(I\left(O_{E}\right)\right) \mathcal{L}\left(\phi / O_{E} ; 1 ; z\right) R[z] \subset \mathbb{T}_{z}\left(E_{\infty}\right) .
$$

Observe that $O_{E}[[z]]=\bigcap_{P} R_{P}[[z]]$. Therefore, we get:

$$
\exp _{\widetilde{\phi}}\left(\psi\left(I\left(O_{E}\right)\right) \mathcal{L}\left(\phi / O_{E} ; 1 ; z\right) O_{E}[z]\right) \subset O_{E}[[z]] \cap \mathbb{T}_{z}\left(E_{\infty}\right)=O_{E}[z]
$$

Thus, we get the first assertion.

Now, let's assume that $S_{E}=\emptyset$. We have:

$$
\bigcap_{P} U\left(\widetilde{\phi} / R_{P}[[z]]\right)=\left\{x \in E_{\infty}[[z]], \exp _{\widetilde{\phi}}(x) \in O_{E}[[z]]\right\}
$$

By 4), we get:

$$
\prod_{P}\left(1-\frac{z^{\operatorname{deg} P}}{\psi(P)} \sigma_{P, O_{E}}\right)\left\{x \in E_{\infty}[[z]], \exp _{\widetilde{\phi}}(x) \in O_{E}[[z]]\right\}=O_{E}[[z]] .
$$

Thus:

$$
\left\{x \in E_{\infty}[[z]], \exp _{\widetilde{\phi}}(x) \in O_{E}[[z]]\right\}=\mathcal{L}\left(\phi / O_{E} ; 1 ; z\right) O_{E}[[z]]
$$

Hence:

$$
U(\widetilde{\phi} / R[z])=\mathcal{L}\left(\phi / O_{E} ; 1 ; z\right) O_{E}[[z]] \cap \mathbb{T}_{z}\left(E_{\infty}\right) .
$$

Since $\mathcal{L}\left(\phi / O_{E} ; 1 ; z\right) \in\left(\mathbb{T}_{z}\left(H_{\infty}\right)[G]\right)^{\times}$, we have:

$$
\mathcal{L}\left(\phi / O_{E} ; 1 ; z\right) O_{E}[[z]] \cap \mathbb{T}_{z}\left(E_{\infty}\right)=\mathcal{L}\left(\phi / O_{E} ; 1 ; z\right) O_{E}[z] .
$$

This concludes the proof of the theorem. 


\subsection{A class formula à la Taelman.}

Recall that ev : $\mathbb{T}_{z}\left(E_{\infty}\right) \rightarrow E_{\infty}$ is the evaluation at $z=1$.

Definition 3.9. We define the equivariant A-harmonic series $\mathcal{L}\left(\phi / O_{E}\right)$ attached to $\phi / O_{E}$ by:

$$
\mathcal{L}\left(\phi / O_{E}\right)=\operatorname{ev}\left(\mathcal{L}\left(\phi / O_{E} ; 1 ; z\right)\right) \in\left(H_{\infty}[G]\right)^{\times} .
$$

Note that:

$$
\mathcal{L}\left(\phi / O_{E}\right)=\prod_{P}\left(1-\frac{1}{\psi(P)} \sigma_{P, O_{E}}\right)^{-1}=\sum_{I \in \mathcal{I}(A), I \subset A} \frac{1}{\psi(I)} \sigma_{I, O_{E}} .
$$

Theorem 3.10. We have:

$$
\alpha_{A}\left(\phi / O_{E}\right)=1
$$

i.e.

$$
\zeta_{O_{E}}(1)=\left[O_{E}: U\left(\phi / O_{E}\right)\right]_{A}\left[H\left(\phi / O_{E}\right)\right]_{A}
$$

Furthermore:

$$
\psi\left(I\left(O_{E}\right)\right) \mathcal{L}\left(\phi / O_{E}\right) O_{E} \subset U_{S t}\left(\phi / O_{E}\right),
$$

and

$$
\left[\frac{U_{S t}\left(\phi / O_{E}\right)}{\psi\left(I\left(O_{E}\right)\right) \mathcal{L}\left(\phi / O_{E}\right) O_{E}}\right]_{A}=\left[\phi\left(\frac{O_{E}}{I\left(O_{E}\right) O_{E}}\right)\right]_{A} .
$$

Proof.

1) Let $J \subset I\left(O_{E}\right)$ be a finite product of maximal ideals of $A$. Set:

$$
\begin{aligned}
\mathcal{L}_{J}\left(\phi / O_{E}\right) & :=\prod_{P}\left(1-\frac{1}{\psi(P)} \sigma_{P, O_{E}}\right)^{-1} \in\left(H_{\infty}[G]\right)^{\times}, \\
\mathcal{L}_{J}\left(\phi / O_{E} ; 1 ; z\right) & :=\prod_{P}\left(1-\frac{z^{\operatorname{deg} P}}{\psi(P)} \sigma_{P, O_{E}}\right)^{-1} \in\left(\mathbb{T}_{z}\left(H_{\infty}\right)[G]\right)^{\times},
\end{aligned}
$$

where $P$ runs through the maximal ideals of $A$ that do not divide $J$.

By Lemma 3.7 and the proof of Theorem 3.8, we have:

$$
\left\{x \in E[[z]], \exp _{\widetilde{\phi}}(x) \in \psi(J) O_{E}[[z]]\right\}=\psi(J) \mathcal{L}_{J}\left(\phi / O_{E} ; 1 ; z\right) O_{E}[[z]] .
$$

We can conclude as in the proof of Theorem 3.8 that:

$$
\psi(J) \mathcal{L}_{J}\left(\phi / O_{E} ; 1 ; z\right) O_{E}[z]=\left\{x \in \mathbb{T}_{z}\left(E_{\infty}\right), \exp _{\widetilde{\phi}}(x) \in \psi(J) O_{E}[z]\right\} .
$$

Therefore, we have a short exact sequence of $A$-modules:

$$
\begin{aligned}
0 \rightarrow & \frac{U\left(\widetilde{\phi} / O_{E}[z]\right)}{\psi(J) \mathcal{L}_{J}\left(\phi / O_{E} ; 1 ; z\right) O_{E}[z]} \rightarrow \widetilde{\phi}\left(\frac{O_{E}[z]}{\psi(J) O_{E}[z]}\right) \rightarrow \\
& \rightarrow \frac{\mathbb{T}_{z}\left(E_{\infty}\right)}{\psi(J) O_{E}[z]+\exp _{\tilde{\phi}}\left(\mathbb{T}_{z}\left(E_{\infty}\right)\right)} \rightarrow H\left(\widetilde{\phi} / O_{E}[z]\right) \rightarrow 0 .
\end{aligned}
$$

Note that $\widetilde{\phi}\left(\frac{O_{E}[z]}{\psi(J) O_{E}[z]}\right)$ is a finitely generated and free $\mathbb{F}_{q}[z]$-module. Let $\rho$ be the Drinfeld module defined over $O_{E}$ such that:

$$
\exp _{\rho}=\psi(J)^{-1} \exp _{\phi} \psi(J) \text {. }
$$

Then, the map $x \mapsto \psi(J)^{-1} x$ induces an isomorphism of $A$-modules (the left module is an $A$-module via $\phi$ and the right module is an $A$-module via $\rho)$ :

$$
\frac{\mathbb{T}_{z}\left(E_{\infty}\right)}{\psi(J) O_{E}[z]+\exp _{\tilde{\phi}}\left(\mathbb{T}_{z}\left(E_{\infty}\right)\right)} \simeq H\left(\widetilde{\rho} / O_{E}[z]\right) .
$$


Observe that ker ev $=(z-1) \mathbb{T}_{z}\left(E_{\infty}\right)$. Furthermore, since $O_{E}[z] \cap(z-1) \mathbb{T}_{z}\left(E_{\infty}\right)=$ $(z-1) O_{E}[z]$, we have :

$$
\begin{gathered}
U\left(\widetilde{\phi} / O_{E}[z]\right) \cap \operatorname{ker} \mathrm{ev}=(z-1) U\left(\widetilde{\phi} / O_{E}[z]\right) \\
\psi(J) \mathcal{L}_{J}\left(\phi / O_{E} ; 1 ; z\right) O_{E}[z] \cap \operatorname{ker} \mathrm{ev}=(z-1) \psi(J) \mathcal{L}_{J}\left(\phi / O_{E} ; 1 ; z\right) O_{E}[z]
\end{gathered}
$$

Thus, the evaluation at $z=1$ induces the following exact sequence of $A$-modules:

$0 \rightarrow(z-1) \frac{U\left(\tilde{\phi} / O_{E}[z]\right)}{\psi(J) \mathcal{L}_{J}\left(\phi / O_{E} ; 1 ; z\right) O_{E}[z]} \rightarrow \frac{U\left(\tilde{\phi} / O_{E}[z]\right)}{\psi(J) \mathcal{L}_{J}\left(\phi / O_{E} ; 1 ; z\right) O_{E}[z]} \rightarrow \frac{U_{S t}\left(\phi / O_{E}\right)}{\psi(J) \mathcal{L}_{J}\left(\phi / O_{E}\right) O_{E}} \rightarrow 0$.

Note also that the evaluation at $z=1$ induces a sequence of $A$-modules:

$$
0 \rightarrow(z-1) \widetilde{\phi}\left(\frac{O_{E}[z]}{\psi(J) O_{E}[z]}\right) \rightarrow \widetilde{\phi}\left(\frac{O_{E}[z]}{\psi(J) O_{E}[z]}\right) \rightarrow \phi\left(\frac{O_{E}}{\psi(J) O_{E}}\right) \rightarrow 0 .
$$

For an $\mathbb{F}_{q}[z]$-module $M$, we denote by $M[z-1]$ the $(z-1)$-torsion. By (3.3), (3.4), (3.5) and the Snake Lemma, we get the following exact sequence of finite $A$-modules:

$$
\begin{aligned}
0 \rightarrow H\left(\widetilde{\rho} / O_{E}[z]\right)[z-1] & \rightarrow H\left(\widetilde{\phi} / O_{E}[z]\right)[z-1] \rightarrow \frac{U_{S t}\left(\phi / O_{E}\right)}{\psi(J) \mathcal{L}_{J}\left(\phi / O_{E}\right) O_{E}} \rightarrow \\
& \rightarrow \phi\left(\frac{O_{E}}{\psi(J) O_{E}}\right) \rightarrow H\left(\rho / O_{E}\right) \rightarrow H\left(\phi / O_{E}\right) \rightarrow 0 .
\end{aligned}
$$

By the proof of Theorem 2.7 we have:

$$
\begin{aligned}
& {\left[H\left(\widetilde{\rho} / O_{E}[z]\right)[z-1]\right]_{A}=\left[H\left(\rho / O_{E}\right)\right]_{A},} \\
& {\left[H\left(\widetilde{\phi} / O_{E}[z]\right)[z-1]\right]_{A}=\left[H\left(\phi / O_{E}\right)\right]_{A} .}
\end{aligned}
$$

Thus:

$$
\left[\frac{U_{S t}\left(\phi / O_{E}\right)}{\psi(J) \mathcal{L}_{J}\left(\phi / O_{E}\right) O_{E}}\right]_{A}=\left[\phi\left(\frac{O_{E}}{J O_{E}}\right)\right]_{A}
$$

2) Now, we have:

$$
\left[O_{E}: \mathcal{L}_{J}\left(\phi / O_{E}\right) O_{E}\right]_{A}=\frac{\operatorname{det}_{K_{\infty}} \mathcal{L}_{J}\left(\phi / O_{E}\right)}{\operatorname{sgn}\left(\operatorname{det}_{K_{\infty}} \mathcal{L}_{J}\left(\phi / O_{E}\right)\right)}
$$

Thus:

$$
\left[O_{E}: \psi(J) \mathcal{L}_{J}\left(\phi / O_{E}\right) O_{E}\right]_{A}=\left[\frac{O_{E}}{J O_{E}}\right]_{A} \frac{\operatorname{det}_{K_{\infty}} \mathcal{L}_{J}\left(\phi / O_{E}\right)}{\operatorname{sgn}\left(\operatorname{det}_{K_{\infty}} \mathcal{L}_{J}\left(\phi / O_{E}\right)\right)} .
$$

And finally, we get:

$$
\left[O_{E}: U_{S t}\left(\phi / O_{E}\right)\right]_{A}=\frac{\left[\frac{O_{E}}{J O_{E}}\right]_{A}}{\left[\phi\left(\frac{O_{E}}{J O_{E}}\right)\right]_{A}} \frac{\operatorname{det}_{K_{\infty}} \mathcal{L}_{J}\left(\phi / O_{E}\right)}{\operatorname{sgn}\left(\operatorname{det}_{K_{\infty}} \mathcal{L}_{J}\left(\phi / O_{E}\right)\right)} .
$$

Set:

$$
L_{J}=\prod_{\mathfrak{P} \mid J} \frac{\left[\frac{O_{E}}{\mathfrak{P}}\right]_{A}}{\left[\phi\left(\frac{O_{E}}{\mathfrak{P}}\right)\right]_{A}}
$$

Then:

$$
\left[O_{E}: U_{S t}\left(\phi / O_{E}\right)\right]_{A}=L_{J} \frac{\operatorname{det}_{K_{\infty}} \mathcal{L}_{J}\left(\phi / O_{E}\right)}{\operatorname{sgn}\left(\operatorname{det}_{K_{\infty}} \mathcal{L}_{J}\left(\phi / O_{E}\right)\right)}
$$


3) Let $N \geq 1$, and we define $J_{N}$ to be the l.c.m. of the product of all maximal ideals of degree $\leq N$ and $I\left(O_{E}\right)$. We have:

$$
\begin{gathered}
\lim _{N \rightarrow+\infty} L_{J_{N}}=L_{A}\left(\phi / O_{E}\right), \\
\lim _{N \rightarrow+\infty} \mathcal{L}_{J_{N}}\left(\phi / O_{E}\right)=1 .
\end{gathered}
$$

In particular:

$$
\lim _{N \rightarrow+\infty} \operatorname{det}_{K_{\infty}} \mathcal{L}_{J_{N}}\left(\phi / O_{E}\right)=1
$$

Thus:

$$
\left[O_{E}: U_{S t}\left(\phi / O_{E}\right)\right]_{A}=L_{A}\left(\phi / O_{E}\right) .
$$

If we apply Theorem 2.7 and Proposition 3.4, we get:

$$
\zeta_{O_{E}}(1)=\left[O_{E}: U\left(\phi / O_{E}\right)\right]_{A}\left[H\left(\phi / O_{E}\right)\right]_{A} .
$$

\section{Log-Algebraicity Theorem}

\subsection{A refinement of Anderson's log-algebraicity theorem.}

We keep the notation of the previous sections.

Lemma 4.1. Let $E / K$ be a finite separable extension, $H \subset E$. Let $P$ be a maximal ideal of $A$ which is unramified in $E$. Let $\lambda_{P} \in \bar{K} \backslash\{0\}$ be a root of $\phi_{P}$. Then:

$$
O_{E\left(\lambda_{P}\right)}=O_{E}\left[\lambda_{P}\right] \text {. }
$$

Proof. Let $F=E\left(\lambda_{P}\right)$. Recall that $F / E$ is a finite abelian extension unramified outside $P, \infty$, and totally ramified at $P$ ([19], Proposition 7.5.18). We also have:

$$
[F: E]=q^{\operatorname{deg} P}-1 .
$$

Write: $\phi_{P}=\sum_{k=0}^{\operatorname{deg} P} \phi_{P, k} \tau^{k}, \phi_{P, k} \in B \subset O_{E}$. Recall that $\phi_{P, 0}=\psi(P)$ and $\phi_{P, \operatorname{deg} P}=1$. Furthermore, $P$ is unramified in $E / K$ and:

$$
\psi(P) O_{E}=P O_{E} .
$$

Let:

$$
G(X)=\sum_{k=0}^{\operatorname{deg} P} \phi_{P, k} X^{q^{k}-1} \in O_{E}[X] .
$$

Then, for any maximal ideal $\mathfrak{P}$ of $O_{E}$ above $P$ :

$$
G(X) \equiv X^{q^{\operatorname{deg} P}-1} \quad(\bmod \mathfrak{P}) .
$$

This implies that $G(X)$ is an Eisenstein polynomial at $\mathfrak{P}$ for every maximal ideal of $O_{E} \mathfrak{P}$ above $P$. Furthermore:

$$
X G^{\prime}(X)+G(X)=\psi(P) .
$$

Therefore:

$$
N_{F / E}\left(G^{\prime}\left(\lambda_{P}\right)\right) O_{E}=P^{q^{\operatorname{deg} P}-2} O_{E} .
$$

But $P^{q^{\operatorname{deg} P}-2} O_{E}$ is the discriminant of $O_{F} / O_{E}$. Thus $O_{F}=O_{E}\left[\lambda_{P}\right]$. 
Let $E / K$ be a finite abelian extension, $H \subset E$. Let $n \geq 0$ be an integer, let $X_{1}, \ldots, X_{n}$ be $n$ indeterminates over K. Let $\tau: E\left[X_{1}, \ldots, X_{n}\right][[z]] \rightarrow E\left[X_{1}, \ldots, X_{n}\right][[z]]$ be the $\mathbb{F}_{q}[[z]]$-homomorphism continuous for the $z$-adic topology such that:

$$
\forall f \in E\left[X_{1}, \ldots, X_{n}\right], \quad \tau(f)=f^{q} .
$$

For a non-zero ideal $I$ of $A$ and for $f=\sum_{i_{1}, \ldots, i_{n} \in \mathbb{N}} f_{i_{1}, \ldots, i_{n}} X_{1}^{i_{1}} \cdots X_{n}^{i_{n}} \in E\left[X_{1}, \ldots, X_{n}\right]$, with $f_{i_{1}, \ldots, i_{n}} \in E$, we set:

$$
I *_{E} f=\sum_{i_{1}, \ldots, i_{n} \in \mathbb{N}} \sigma_{I, O_{E}}\left(f_{i_{1}, \ldots, i_{n}}\right) \phi_{I}\left(X_{1}\right)^{i_{1}} \cdots \phi_{I}\left(X_{n}\right)^{i_{n}},
$$

where $\sigma_{I, O_{E}}$ is defined in Section 3.4. Recall that $I\left(O_{E}\right)$ is the product of maximal ideals of $A$ that are wildly ramified in $E / K$.

Theorem 4.2. For all $f \in O_{E}\left[X_{1}, \ldots, X_{n}\right]$, we have:

$$
\exp _{\widetilde{\phi}}\left(\psi\left(I\left(O_{E}\right)\right) \sum_{I \in \mathcal{I}(A), I \subset A} \frac{I *_{E} f}{\psi(I)} z^{\operatorname{deg} I}\right) \in O_{E}\left[X_{1}, \ldots, X_{n}, z\right] .
$$

In particular, for all $f \in B\left[X_{1}, \ldots, X_{n}\right]$, we have:

$$
\exp _{\widetilde{\phi}}\left(\sum_{I \in \mathcal{I}(A), I \subset A} \frac{I *_{H} f}{\psi(I)} z^{\operatorname{deg} I}\right) \in B\left[X_{1}, \ldots, X_{n}, z\right] .
$$

Remark 4.3. This result is a generalization of the Log-Algebraicity Theorems established in [1], 2] (in these papers the theorem is proved for $E=H, d_{\infty}=1$ and $n \leq 1)$. Furthermore, the result in the case $E=H$ can be proved along the same lines as that used to prove [2, Theorem 3. Following 9], Section 2.6, we will show below how Theorem 3.8 implies the Log-Algebraicity Theorem. Observe also that the case $n=0$ is a direct consequence of Theorem 3.8 .

Proof. Let's write:

$$
\exp _{\tilde{\phi}}\left(\psi\left(I\left(O_{E}\right)\right) \sum_{I} \frac{I *_{E} f}{\psi(I)} z^{\operatorname{deg} I}\right)=\sum_{m \geq 0} g_{m}\left(X_{1}, \ldots, X_{n}\right) z^{m},
$$

with $g_{m}\left(X_{1}, \ldots, X_{n}\right) \in E\left[X_{1}, \ldots, X_{n}\right]$.

1) Let $P_{1}, \ldots, P_{n}$ be $n$ distinct maximal ideals of $A$ which are unramified in $E$, with $q^{\operatorname{deg} P_{i}} \geq 3, i=1, \ldots, n$, and for $i=1, \ldots, n$, let $\lambda_{i} \neq 0$ be a root of $\phi_{P_{i}}$. Set:

$$
F=E\left(\lambda_{1}, \ldots, \lambda_{n}\right) .
$$

Then $F / E$ is unramified outside $P_{1}, \ldots, P_{n}, \infty, F / K$ a finite abelian extension of $K$ which is tamely ramified at $P_{1}, \ldots, P_{n}$. Let $O_{F}$ be the integral closure of $A$ in $F$. Let $Q$ be any maximal ideal of $A$, if $Q$ is not wildly ramified in $E$, we have ([19], Proposition 7.5.4):

and:

$$
\sigma_{Q, O_{F}}\left(\lambda_{i}\right)=\phi_{Q}\left(\lambda_{i}\right), \quad \text { if } \quad Q \neq P_{i},
$$

$$
\sigma_{P_{i}, O_{F}}\left(\lambda_{i}\right)=0
$$

We deduce that:

$$
\left.\psi\left(I\left(O_{E}\right)\right) \sum_{I} \frac{I *_{E} f}{\psi(I)} z^{\operatorname{deg} I}\right|_{X_{i}=\lambda_{i}}=\psi\left(I\left(O_{F}\right)\right) \mathcal{L}\left(\phi / O_{F} ; 1 ; z\right) f\left(\lambda_{1}, \ldots, \lambda_{n}\right) .
$$


Therefore, by Theorem 3.8, we get:

$$
\forall m \geq 0, \quad g_{m}\left(\lambda_{1}, \ldots, \lambda_{n}\right) \in O_{F} .
$$

Let $i \in\{1, \ldots, n\}$. Then:

$$
E\left(\lambda_{i}\right) \cap E\left(\lambda_{1}, \ldots \lambda_{i-1}, \lambda_{i+1}, \ldots, \lambda_{n}\right)=E .
$$

Furthermore, the discriminant of $O_{E\left(\lambda_{i}\right)} / O_{E}$ and $O_{E\left(\lambda_{1}, \ldots \lambda_{i-1}, \lambda_{i+1}, \ldots, \lambda_{n}\right)} / O_{E}$ are relatively prime, thus, by Lemma 4.1, we have:

$$
O_{F}=O_{E}\left[\lambda_{1}, \ldots, \lambda_{n}\right]
$$

Finally, for $m \geq 0$, for $n$ distinct maximal ideals $P_{1}, \ldots, P_{n}$ of $A$ that are unramified in $E / K$, with $q^{\operatorname{deg} P_{i}} \geq 3, i=1, \ldots, n$, and for $i=1, \ldots, n$, if $\lambda_{i} \neq 0$ be a root of $\phi_{P_{i}}$, then we have:

$$
g_{m}\left(\lambda_{1}, \ldots, \lambda_{n}\right) \in O_{E}\left[\lambda_{1}, \ldots, \lambda_{n}\right]
$$

This implies:

$$
\forall m \geq 0, \quad g_{m}\left(X_{1}, \ldots, X_{n}\right) \in O_{E}\left[X_{1}, \ldots, X_{n}\right] .
$$

2) We fix a $K$-embedding of $\bar{K}$ in $\mathbb{C}_{\infty}$. For $\sigma \in \operatorname{Gal}(H / K)$, let $\Lambda\left(\phi^{\sigma}\right) \subset \mathbb{C}_{\infty}$ be the $A$-module of periods of $\phi^{\sigma}$, and let $\Lambda\left(\phi^{\sigma}\right) K_{\infty}$ be the $K_{\infty}$-vector space generated by $\Lambda\left(\phi^{\sigma}\right)$. Then $\frac{\Lambda\left(\phi^{\sigma}\right) K_{\infty}}{\Lambda\left(\phi^{\sigma}\right)}$ is compact, thus there exists a constant $C \in \mathbb{R}$ such that:

$$
\forall \sigma \in \operatorname{Gal}(H / K), \forall x \in \Lambda\left(\phi^{\sigma}\right) K_{\infty}, \quad v_{\infty}\left(\exp _{\phi^{\sigma}}(x)\right) \geq C .
$$

Recall that, if $\sigma \in \operatorname{Gal}(H / K)$, then there exists a non-zero ideal $J$ of $A$ such that $\sigma=(J, H / K)=\sigma_{J}$, and we have ([19], Theorem 7.4.8):

$$
\phi_{J} \phi_{a}=\phi_{a}^{\sigma} \phi_{J} .
$$

Thus:

In particular:

$$
\exp _{\phi^{\sigma}} \psi(J)=\phi_{J} \exp _{\phi} .
$$

$$
\begin{gathered}
\Lambda\left(\phi^{\sigma}\right)=\psi(J) J^{-1} \Lambda(\phi), \\
\Lambda\left(\phi^{\sigma}\right) K_{\infty}=\psi(J) \Lambda(\phi) K_{\infty} .
\end{gathered}
$$

Therefore, there exists a constant $C^{\prime} \in \mathbb{R}$, such that:

$\forall \sigma \in \operatorname{Gal}(H / K), \forall x_{1}, \ldots, x_{n} \in \Lambda\left(\phi^{\sigma}\right) K_{\infty}, \forall I \in \mathcal{I}(A), \quad v_{\infty}\left(\left.I *_{E}^{\sigma} f^{\sigma}\right|_{X_{i}=\exp _{\phi} \sigma\left(x_{i}\right)}\right) \geq C^{\prime}$, where $*_{E}^{\sigma}$ is the map $*$ attached to $\phi^{\sigma}$. Now, recall that $\exp _{\phi}=\sum_{j \geq 0} e_{j}(\phi) \tau^{j}$, then there exists a constant $C^{\prime \prime}>0$ such that (Lemma 3.7):

$$
\forall \sigma \in \operatorname{Gal}(H / K), \forall j \gg 0, \quad v_{\infty}\left(e_{j}\left(\phi^{\sigma}\right)\right) \geq C^{\prime \prime} j q^{j} .
$$

Note also that there exists $C^{\prime \prime \prime} \in \mathbb{R}$ such that:

$$
\forall \sigma \in \operatorname{Gal}(H / K), \forall I \in \mathcal{I}(A), \operatorname{deg} I=m \gg 0, \quad v_{\infty}\left(\frac{1}{\sigma(\psi(I))}\right) \geq \frac{m}{d_{\infty}}+C^{\prime \prime \prime} .
$$

This implies that there exists an integer $m_{0} \in \mathbb{N}$, such that:

$\forall m \geq m_{0}, \forall \sigma \in \operatorname{Gal}(E / K), \forall \lambda_{1}, \ldots, \lambda_{n} \in \exp _{\phi^{\sigma}}\left(\Lambda\left(\phi^{\sigma}\right) K_{\infty}\right), \quad v_{\infty}\left(g_{m}^{\sigma}\left(\lambda_{1}, \ldots, \lambda_{n}\right)\right)>0$.

3) Let $m_{0} \in \mathbb{N}$ be as in 2). Let $\lambda_{1}, \ldots, \lambda_{n}$ be $n$ torsion points for $\phi$. Let $F=$ $E\left(\lambda_{1}, \ldots, \lambda_{n}\right)$. Then $F / K$ is a finite abelian extension. Let $w$ be a place of $F$ above $\infty$. Let $i_{w}: E \rightarrow \mathbb{C}_{\infty}$ be the $K$-embedding of $F$ in $\mathbb{C}_{\infty}$ corresponding to $w$. Then there exists $\sigma \in \operatorname{Gal}(F / K)$ such that:

$$
\forall m \geq 0, \quad i_{w}\left(g_{m}\left(\lambda_{1}, \ldots, \lambda_{n}\right)\right)=\sigma\left(g_{m}\left(\lambda_{1}, \ldots, \lambda_{n}\right)\right)=g_{m}^{\sigma}\left(\sigma\left(\lambda_{1}\right), \ldots, \sigma\left(\lambda_{n}\right)\right) .
$$


Observe that $\sigma\left(\lambda_{i}\right) \in \exp _{\phi^{\sigma}}\left(\Lambda\left(\phi^{\sigma}\right) K_{\infty}\right), i=1, \ldots, n$ ([19], Proposition 7.5.16). Therefore:

$$
\forall m \geq m_{0}, \quad w\left(g_{m}\left(\lambda_{1}, \ldots, \lambda_{n}\right)\right)>0 .
$$

Thus, we get that for any place $w$ of $F$ above $\infty$ :

$$
\forall m \geq m_{0}, \quad w\left(g_{m}\left(\lambda_{1}, \ldots, \lambda_{n}\right)\right)>0 .
$$

But by 1$), \forall m \geq 0, g_{m}\left(\lambda_{1}, \ldots, \lambda_{n}\right) \in O_{F}$. Since $O_{F}$ is the set of elements of $F$ which are regular outside the places of $F$ above $\infty$, we deduce that:

$$
\forall m \geq m_{0}, \quad g_{m}\left(\lambda_{1}, \ldots, \lambda_{n}\right)=0 .
$$

And the above property is true for any $n$ torsion points of $\phi$, thus:

$$
\forall m \geq m_{0}, \quad g_{m}\left(X_{1}, \ldots, X_{n}\right)=0 .
$$

M. Papanikolas informed us that, together with N. Green, they obtained explicit formulas for Anderson's Log-Algebraicity Theorem ([1, Theorem 5.1.1) when the genus $g$ of $K$ is one and $d_{\infty}=1$.

\subsection{Several variable $L$-series and shtukas.}

In this section, we present an alternative approach to the several variable LogAlgebraicity Theorem (Theorem 4.2) by using the seminal works of Drinfeld [12], [13, 14] (see also [1, 27, and [19, Chapter 6).

We recall some notation for the convenience of the reader. Let $X / \mathbb{F}_{q}$ be a smooth projective geometrically irreducible curve of genus $g$ whose function field is $K$. We will consider $\infty$ as a closed point of $X$ of degree $d_{\infty}$. Recall that $K_{\infty}$ is the completion of $K$ at $\infty, \bar{K}_{\infty}$ is a fixed algebraic closure of $K_{\infty}$, and $\mathbb{C}_{\infty}$ is the completion of $\bar{K}_{\infty}$. Let $\operatorname{sgn}: K_{\infty}^{\times} \rightarrow \mathbb{F}_{\infty}^{\times}$be a sign function $\left(\mathbb{F}_{\infty}\right.$ is the residue field of $K_{\infty}$ and $d_{\infty}=\left[\mathbb{F}_{\infty}: \mathbb{F}_{q}\right]$ ), i.e. $\operatorname{sgn}$ is a group homomorphism such that $\left.\operatorname{sgn}\right|_{\mathbb{F}_{\infty}^{\times}}=\left.\operatorname{Id}\right|_{\mathbb{F}_{\infty}^{\times}}$. We fix $\pi \in K \cap \operatorname{Ker}(\operatorname{sgn})$ and such that $K_{\infty}=\mathbb{F}_{\infty}((\pi))$.

We set $\bar{X}=X \otimes_{\mathbb{F}_{q}} \mathbb{C}_{\infty}$, and $\bar{A}:=A \otimes_{\mathbb{F}_{q}} \mathbb{C}_{\infty}$. Then $F:=\operatorname{Frac}(\bar{A})$ is the function field of $\bar{X}$. We identify $\mathbb{C}_{\infty}$ with its image $1 \otimes \mathbb{C}_{\infty}$ in $F$. Note that $\bar{A}$ is the set of elements of $F / \mathbb{C}_{\infty}$ which are "regular outside $\infty$ ". We denote by $\tau: F \rightarrow F$ the $K$-algebra homomorphism such that:

$$
\left.\tau\right|_{\bar{A}}=\operatorname{Id}_{A} \otimes \operatorname{Frob}_{\mathbb{C}_{\infty}} .
$$

For $m \geq 0$, we also set:

$$
\forall x \in F, \quad x^{(m)}=\tau^{m}(x) .
$$

Let $P$ be a point of $\bar{X}\left(\mathbb{C}_{\infty}\right)$. We denote by $P^{(i)}$ the point of $\bar{X}\left(\mathbb{C}_{\infty}\right)$ obtained by applying $\tau^{i}$ to the coordinates of $P$. If $D \in \operatorname{Div}(\bar{X}), D=\sum_{j=1}^{n} n_{P_{j}}\left(P_{j}\right), P_{j} \in$ $\bar{X}\left(\mathbb{C}_{\infty}\right), n_{P_{j}} \in \mathbb{Z}$, we set:

$$
D^{(i)}=\sum_{j=1}^{n} n_{P_{j}}\left(P_{j}^{(i)}\right)
$$

If $D=(x), x \in F^{\times}$, then:

$$
D^{(i)}=\left(x^{(i)}\right)
$$


We fix a point $\bar{\infty}$ of $X\left(\mathbb{C}_{\infty}\right)$ above $\infty$. Let $\xi$ be the point of $\bar{X}\left(\mathbb{C}_{\infty}\right)$ corresponding to the kernel of the map $\bar{A} \rightarrow \mathbb{C}_{\infty}, \sum x_{i} \otimes a_{i} \mapsto \sum x_{i} a_{i}$. Let $\rho: K \rightarrow K \otimes 1, x \mapsto x \otimes 1$. Then:

$$
F=\mathbb{C}_{\infty}(\rho(K))
$$

By [27] (see also [19], section 7.11), there exists a function $f \in F^{\times}$, such that:

$$
V^{(1)}-V+(\xi)-(\bar{\infty})=(f)
$$

for some effective divisor $V$ of $\bar{X} / \mathbb{C}_{\infty}$ of degree $g$. The points $\xi$ and $\bar{\infty}^{(-1)}$ do not belong to the support of $V([27$, Corollary 0.3 .3$)$. We identify the completion of $F$ at $\bar{\infty}$ with:

$$
\mathbb{C}_{\infty}\left(\left(\frac{1}{t}\right)\right)
$$

where $t=\rho\left(\pi^{-1}\right)$. We have a natural sign function $\overline{\operatorname{sgn}}: \mathbb{C}_{\infty}\left(\left(\frac{1}{t}\right)\right)^{\times} \rightarrow \mathbb{C}_{\infty}^{\times}$attached to $\frac{1}{t}$. We normalize $f$ such that $\overline{\operatorname{sgn}}(f)=1$.

We set:

$$
\begin{gathered}
(\infty)=\sum_{i=0}^{d_{\infty}-1}\left(\bar{\infty}^{(i)}\right) \\
W\left(\mathbb{C}_{\infty}\right)=\bigcup_{m \geq 0} L(V+m(\infty)),
\end{gathered}
$$

where:

$$
L(V+m(\infty))=\left\{x \in F^{\times},(x)+V+m(\infty) \geq 0\right\} \cup\{0\} .
$$

Observe that for $i>0$ :

$$
\left(f f^{(1)} \cdots f^{(i-1)}\right)=V^{(i)}-V+(\xi)+\cdots+\left(\xi^{(i-1)}\right)-\sum_{k=0}^{i-1}\left(\bar{\infty}^{(k)}\right) .
$$

We have (see for example [27], paragraph 0.3.5):

$$
W\left(\mathbb{C}_{\infty}\right)=\oplus_{i \geq 0} \mathbb{C}_{\infty} f \cdots f^{(i-1)} .
$$

If $L$ is a sub- $\mathbb{F}_{q}$-algebra of $\mathbb{C}_{\infty}$, we set:

$$
W(L)=\oplus_{i \geq 0} L f \cdots f^{(i-1)} .
$$

Let $a \in A$, then we can write:

$$
\rho(a)=a \otimes 1=\sum_{i=0}^{\operatorname{deg} a} \phi_{a, i} f \cdots f^{(i-1)},
$$

where $\phi_{a, i} \in \mathbb{C}_{\infty}$, and:

$$
\begin{gathered}
\phi_{a, \operatorname{deg} a}=\overline{\operatorname{sgn}}(a), \\
\phi_{a, 0}=a .
\end{gathered}
$$

In particular, note that $\bar{\infty}$ does not belong to the support of $V$. The map $\phi: A \rightarrow$ $\mathbb{C}_{\infty}\{\tau\}$ such that:

$$
\forall a \in A, \quad \phi_{a}=\sum \phi_{a, i} \tau^{i}
$$

is a sign-normalized rank one Drinfeld module by the Drinfeld correspondence attached to $f([27]$, paragraph 0.3.5, see also [19], section 7.11$)$. Let's write:

$$
\exp _{\phi}=\sum e_{i}(\phi) \tau^{i}, \quad e_{i}(\phi) \in \mathbb{C}_{\infty}
$$


We have ([27, Proposition 0.3.6):

$$
\forall i \geq 0, \quad e_{i}(\phi)=\frac{1}{\left.f \cdots f^{(i-1)}\right|_{\xi^{(i)}}} .
$$

Let $\mathbb{H}=\operatorname{Frac}(A \otimes B) \subset F$. By Drinfeld's correspondence (see [19], Chapter 6), $f \in \mathbb{H}$. Thus:

$$
f=t+\sum_{i \geq 0} f_{i} \frac{1}{t^{i}} \in H\left(\left(\frac{1}{t}\right)\right) \subset \mathbb{C}_{\infty}\left(\left(\frac{1}{t}\right)\right)
$$

where $f_{i} \in H, \forall i \geq 0$.

We view $\mathbb{H}$ as a function field over $\rho(K)=K \otimes 1$. Let $\mathbb{K}=\operatorname{Frac}(A \otimes A)$. Let $\infty$ be the unique place of $\mathbb{K} / \rho(K)$ which is above the place $\infty$ of $K / \mathbb{F}_{q}$. Then the completion of $\mathbb{K}$ above $\infty$ is:

$$
\mathbb{K}_{\infty}=\rho(K)\left(\mathbb{F}_{\infty}\right)((1 \otimes \pi)) .
$$

Observe that the set of elements of $\mathbb{K} / \rho(K)$ which are regular outside $\infty$ is:

$$
\mathbb{A}:=A[\rho(K)]=K \otimes A .
$$

We set $\mathbb{B}:=B[\rho(K)]=K \otimes B$, then $\mathbb{B}$ is the integral closure of $\mathbb{A}$ in $\mathbb{H}$. Let $G=$ $\operatorname{Gal}(H / K) \simeq \operatorname{Gal}(\mathbb{H} / \mathbb{K})$. Let $\varphi: \mathbb{A} \rightarrow \mathbb{H}\{\tau\}$ be the $\rho(K)$-algebra homomorphism such that:

$$
\forall a \in A, \quad \varphi_{a}=\sum_{i=0}^{\operatorname{deg} a} \phi_{a, i} f \cdots f^{(i-1)} \tau^{i} \in \mathbb{H}\{\tau\} .
$$

Let $\exp _{\varphi} \in \mathbb{H}\{\{\tau\}\}$ be the following element:

$$
\exp _{\varphi}=\sum_{i \geq 0} f \cdots f^{(i-1)} e_{i}(\phi) \tau^{i}=\sum_{i \geq 0} \frac{f \cdots f^{(i-1)}}{\left.f \cdots f^{(i-1)}\right|_{\xi^{(i)}}} \tau^{i} .
$$

Then:

$$
\forall a \in \mathbb{A}, \quad \exp _{\varphi} a=\varphi_{a} \exp _{\varphi} .
$$

Let $\mathbb{H}_{\infty}=\mathbb{H} \otimes_{\mathbb{K}} \mathbb{K}_{\infty}$, then $\exp _{\varphi}$ converges on $\mathbb{H}_{\infty}$.

Let $\mathfrak{P}$ be a maximal ideal of $B$. Then $\mathfrak{P B}$ is a maximal ideal of $\mathbb{B}$. Let $v_{\mathfrak{P}}$ : $\mathbb{H} \rightarrow \mathbb{Z} \cup\{+\infty\}$ be the valuation on $\mathbb{H}$ attached to $\mathfrak{P B}$. Since for all $a \in A$, $\rho(a)=\sum_{j=0}^{\operatorname{deg} a} \phi_{a, j} f \cdots f^{(j-1)}$, we deduce that:

$$
\forall i \geq 0, \quad v_{\mathfrak{P}}\left(f^{(i)}\right)=q^{i} v_{\mathfrak{P}}(f)=0 .
$$

However, we warn the reader that, if $g>0$, we have:

$$
f \notin \mathbb{B} \text {. }
$$

We set:

$$
W(B)=\oplus_{i \geq 0} B f \cdots f^{(i-1)} .
$$

Lemma 4.4.

1) $W(B)$ is a $A \otimes B$-module containing $A \otimes B$, furthermore $W(B)$ is a $A \otimes A$-module via $\varphi$.

2) Let $W(B) \mathbb{B}$ be the $\mathbb{B}$-module generated by $W(B)$. Let $\mathfrak{P}$ be a maximal ideal of $B$. The inclusion $\mathbb{B} \subset W(B) \mathbb{B}$ induces an equality:

$$
\frac{\mathbb{B}}{\mathfrak{P B}}=\frac{W(B) \mathbb{B}}{\mathfrak{P} W(B) \mathbb{B}} .
$$

3) $W(B) \mathbb{B}$ is a fractional ideal of $\mathbb{B}$. In particular, it is discrete in $\mathbb{H}_{\infty}$. 
Proof. We have:

$$
\forall i \geq 0, \forall a \in A, \quad \rho(a) f \cdots f^{(i-1)}=\sum_{j=0}^{\operatorname{deg} a} \phi_{a, j}^{q^{i}} f \cdots f^{(i+j-1)} \in W(B) .
$$

Observe that:

$$
\forall i, j \geq 0, \quad f \cdots f^{(j-1)} \tau^{j}\left(f \cdots f^{(i-1)}\right)=f \cdots f^{(i+j-1)} .
$$

The assertion 1) follows.

We set: $O_{\mathfrak{P}}=\left\{x \in \mathbb{H}, v_{\mathfrak{P}}(x) \geq 0\right\}$. Since $\frac{O_{\mathfrak{P}}}{\mathfrak{P} O_{\mathfrak{P}}} \simeq \frac{\mathbb{B}}{\mathfrak{P} \mathbb{B}}$ and $\mathbb{B} \subset W(B) \mathbb{B} \subset O_{\mathfrak{P}}$, the assertion 2) holds.

Let's prove the assertion 3). Note that $A \otimes H$ is the set of elements of $\mathbb{H}$ which are regular outside $\bar{\infty}$. By the expression (4.1) of the divisor of $f \cdots f^{(i-1)}, i \geq 0$, there exists $a \in A \otimes B \backslash\{0\}$ such that:

$$
\forall i \geq 0, \quad \text { af } \cdots f^{(i-1)} \in A \otimes H .
$$

Since for every maximal ideal $\mathfrak{P}$ of $B$, and for all $i \geq 0, v_{\mathfrak{P}}\left(f \cdots f^{(i-1)}\right)=0$, we deduce that:

$$
\forall i \geq 0, a f \cdots f^{(i-1)} \in A \otimes B
$$

Thus, there exists $a \in \mathbb{B} \backslash\{0\}$ such that $a W(B) \subset \mathbb{B}$. Since $\mathbb{B}$ is discrete in $\mathbb{H}_{\infty}$, we get the desired result.

Let's observe that, by Lemma 4.4 $W(B) \mathbb{B}$ is an $\mathbb{A}$-module via $\varphi$. Let $\mathfrak{P}$ be a maximal ideal of $B$, then, again by Lemma $4.4, \frac{\mathbb{B}}{\mathfrak{P} \mathbb{B}}$ is an $\mathbb{A}$-module via $\varphi$, and we denote this latter $\mathbb{A}$-module by $\varphi\left(\frac{\mathbb{B}}{\mathfrak{B} \mathbb{B}}\right)$.

Lemma 4.5. Let $\mathfrak{P}$ be a maximal ideal of $B$. Then:

$$
\operatorname{Fitt}_{\mathbb{A}} \varphi\left(\frac{\mathbb{B}}{\mathfrak{P B} B}\right)=\left(\left[\frac{B}{\mathfrak{P} B}\right]_{A}-\rho\left(\left[\frac{B}{\mathfrak{P} B}\right]_{A}\right)\right) \mathbb{A} .
$$

Proof. Recall that:

$$
\left[\frac{B}{\mathfrak{P} B}\right]_{A}=\psi\left(P^{e}\right)
$$

where $e=\operatorname{dim}_{\frac{A}{P}} \frac{B}{\mathfrak{P}}$. Set $a A=P^{e}$ where $\operatorname{sgn}(a)=1$. Then:

$$
\rho(a)=\sum \phi_{a, i} f \cdots f^{(i-1)} .
$$

Therefore:

$$
\forall x \in \frac{\mathbb{B}}{\mathfrak{P B}}, \quad \varphi_{a-\rho(a)}(x)=0 .
$$

Thus, by similar arguments to those of [6], Lemma 5.8, we have an $\mathbb{A}$-module isomorphism:

$$
\varphi\left(\frac{\mathbb{B}}{\mathfrak{P B}}\right) \simeq \frac{\mathbb{A}}{(a-\rho(a)) \mathbb{A}} .
$$

If $M$ is an $\mathbb{A}$-module such that $M$ is a finite dimensional $\rho(K)$-vector space and its Fitting ideal is principal, $\operatorname{Fitt}_{\mathbb{A}}(M)=x \mathbb{A}$, then we set:

$$
[M]_{\mathbb{A}}=\frac{x}{\operatorname{sgn}(x)} .
$$


By the above Lemma, we can form the $L$-series attached to $\varphi / W(B)$ :

$$
L(\varphi / W(B))=\prod_{\mathfrak{P}} \frac{\left[\frac{\mathbb{B}}{\mathfrak{P} \mathbb{B}}\right]_{\mathbb{A}}}{\left[\varphi\left(\frac{\mathbb{B}}{\mathfrak{P} \mathbb{B}}\right)\right]_{\mathbb{A}}}=\prod_{\mathfrak{P}}\left(1-\frac{\rho\left(\left[\frac{B}{\mathfrak{P} B}\right]_{A}\right)}{\left[\frac{B}{\mathfrak{P} B}\right]_{A}}\right)^{-1} \in \mathbb{K}_{\infty} \times .
$$

Note that $L(\varphi / W(B))$ is in fact an element in the $\infty$-adic completion of $K_{\infty}[\rho(A)]=$ $A \otimes K_{\infty}$ which is an affinoid algebra over $K_{\infty}$, and $L(\varphi / W(B))$ is a special value of a twisted zeta function (see [5], Section 5.2).

We denote by $\tau: \mathbb{H}_{\infty} \rightarrow \mathbb{H}_{\infty}$ the continuous homomorphism of $\rho(K)$-algebras such that $\forall x \in H_{\infty}, \tau(x)=x^{q}$. Let $z$ be an indeterminate. The map $\tau: \mathbb{H}_{\infty} \rightarrow \mathbb{H}_{\infty}$ extends uniquely into a continuous homomorphism (for the $z$-adic topology) of $\mathbb{F}_{q}[[z]]$-algebras $\tau: \mathbb{H}_{\infty}[[z]] \rightarrow \mathbb{H}_{\infty}[[z]]$. Let $\mathbb{T}_{z}\left(\mathbb{H}_{\infty}\right) \subset \mathbb{H}_{\infty}[[z]]$ be the $\infty$-adic completion of $\mathbb{H}_{\infty}[z]$, i.e. an element $g \in \mathbb{T}_{z}\left(\mathbb{H}_{\infty}\right)$ can be uniquely written $g=$ $\sum_{i \geq 0} g_{i} z^{i}, g_{i} \in \mathbb{H}_{\infty}$, such that $\lim _{i \rightarrow+\infty} g_{i}=0$. We also denote by $\mathbb{T}_{z}\left(\mathbb{K}_{\infty}\right)$ the $\infty$-adic completion of $\mathbb{K}_{\infty}[z]$. Note that $\mathbb{T}_{z}\left(\mathbb{H}_{\infty}\right)$ is a free $\mathbb{T}_{z}\left(\mathbb{K}_{\infty}\right)$-module of rank $[H: K]$, and if $\left(e_{1}, \ldots, e_{n}\right)$ is a $K$-basis of $\left.H(n=[H: K])\right)$, then:

$$
\mathbb{T}_{z}\left(\mathbb{H}_{\infty}\right)=\oplus_{i=1}^{n} e_{i} \mathbb{T}_{z}\left(\mathbb{K}_{\infty}\right) .
$$

Observe also that $G$ acts on $\mathbb{T}_{z}\left(\mathbb{H}_{\infty}\right)$ and $\mathbb{T}_{z}\left(\mathbb{H}_{\infty}\right)$ is a free $\mathbb{T}_{z}\left(\mathbb{K}_{\infty}\right)[G]$-module of rank one by the normal basis Theorem. We denote by $\mathbb{T}_{z}\left(\mathbb{H}_{\infty}\right)[G]$ the ring:

$$
\mathbb{T}_{z}\left(\mathbb{H}_{\infty}\right)[G]:=\oplus_{\sigma \in G} \mathbb{T}_{z}\left(\mathbb{H}_{\infty}\right) \sigma
$$

where the product rule is given by:

$$
\forall \sigma_{1}, \sigma_{2} \in G, \forall g_{1}, g_{2} \in \mathbb{T}_{z}\left(\mathbb{H}_{\infty}\right), \quad\left(g_{1} \sigma_{1}\right)\left(g_{2} \sigma_{2}\right)=g_{1} \sigma_{1}\left(g_{2}\right) \sigma_{1} \sigma_{2} .
$$

Let's set:

$$
\exp _{\widetilde{\varphi}}=\sum_{i \geq 0} \frac{f \cdots f^{(i-1)}}{\left.f \cdots f^{(i-1)}\right|_{\xi^{(i)}}} z^{i} \tau^{i} \in \mathbb{H}[z]\{\{\tau\}\} .
$$

Let $I$ be a non-zero ideal of $A$. We set:

$$
u_{I}=\sum_{i=0}^{\operatorname{deg} I} \phi_{I, i} f \cdots f^{(i-1)} \in W(B),
$$

where $\phi_{I}=\sum_{i=0}^{\operatorname{deg} I} \phi_{I, i} \tau^{i}, \phi_{I, i} \in B$. Note that if $I=a A$, we have:

$$
u_{I}=\frac{\rho(a)}{\operatorname{sgn}(a)} \text {. }
$$

Furthermore, we prove (see [1], Section 3.7 for the case $d_{\infty}=1$ ):

Lemma 4.6. Let $I, J$ be two non-zero ideals of $A$. We have:

$$
\begin{gathered}
\left.u_{I}\right|_{\xi}=\psi(I), \\
\sigma_{I}(f) u_{I}=f \tau\left(u_{I}\right), \\
u_{I J}=\sigma_{I}\left(u_{J}\right) u_{I} .
\end{gathered}
$$

Proof. The fact that $\left.u_{I}\right|_{\xi}=\psi(I)$ comes from the definition of $u_{I}$. Note that we have a natural isomorphism of $B$-modules:

$$
\gamma_{\phi}: W(B) \simeq B\{\tau\}, \quad f \cdots f^{(i-1)} \mapsto \tau^{i} .
$$

In particular:

$$
\begin{gathered}
\forall x \in W(B), \quad \gamma_{\phi}\left(f x^{(1)}\right)=\tau \gamma_{\phi}(x), \\
\forall x \in W(B), \forall a \in A, \quad \gamma_{\phi}(\rho(a) x)=\gamma_{\phi}(x) \phi_{a} .
\end{gathered}
$$


By explicit reciprocity law (see [19], Theorem 7.4.8), we have:

$$
\forall a \in A, \quad \phi_{I} \phi_{a}=\sigma_{I}(\phi)_{a} \phi_{I} .
$$

By direct calculations, we deduce from this:

$$
\sigma_{I}(f) u_{I}=f \tau\left(u_{I}\right),
$$

Now, let $J$ be a non-zero ideal of $A$. We have:

$$
\gamma_{\phi}\left(u_{I J}\right)=\phi_{I J}=\sigma_{I}\left(\phi_{J}\right) \phi_{I}
$$

But, since $\forall i \geq 0, \sigma_{I}\left(f \cdots f^{(i-1)}\right) u_{I}=f \cdots f^{(i-1)} u_{I}^{(i)}$, we have :

$$
\gamma_{\phi}\left(\sigma_{I}\left(u_{J}\right) u_{I}\right)=\sigma_{I}\left(\phi_{J}\right) \phi_{I} .
$$

Thus:

$$
u_{I J}=\sigma_{I}\left(u_{J}\right) u_{I}
$$

We deduce that if $P, Q$ are maximal ideals of $A$ :

$\left(1-\frac{u_{P}}{\psi(P)} z^{\operatorname{deg} P} \sigma_{P}\right)\left(1-\frac{u_{Q}}{\psi(Q)} z^{\operatorname{deg} Q_{\sigma_{Q}}}\right)=\left(1-\frac{u_{Q}}{\psi(Q)} z^{\operatorname{deg}(Q)} \sigma_{Q}\right)\left(1-\frac{u_{P}}{\psi(P)} z^{\operatorname{deg} P} \sigma_{P}\right)$.

For every integer $n \geq 1$, we set:

$$
\left(1-\frac{u_{P}}{\psi(P)^{n}} z^{\operatorname{deg} P} \sigma_{P}\right)^{-1}:=\sum_{k \geq 0} \frac{u_{P^{k}}}{\psi\left(P^{k}\right)^{n}} z^{k \operatorname{deg} P} \sigma_{P^{k}} \in \mathbb{T}_{z}\left(\mathbb{H}_{\infty}\right)[G] .
$$

We define:

$$
\forall n \geq 1, \quad \mathcal{L}(\varphi ; n ; z)=\prod_{P}\left(1-\frac{u_{P}}{\psi(P)^{n}} z^{\operatorname{deg} P} \sigma_{P}\right)^{-1} \in\left(\mathbb{T}_{z}\left(\mathbb{H}_{\infty}\right)[G]\right)^{\times},
$$

where $P$ runs through the maximal ideals of $A$. Note that, for any $n \geq 1, \mathcal{L}(\varphi ; n ; z)$ induces a $\mathbb{T}_{z}\left(\mathbb{K}_{\infty}\right)$-linear endomorphism of $\mathbb{T}_{z}\left(\mathbb{H}_{\infty}\right)$, and we denote by $\operatorname{det}_{\mathbb{T}_{z}\left(\mathbb{K}_{\infty}\right)} \mathcal{L}(\varphi ; n ; z)$ its determinant. Let's set:

$$
W(B[z])=\oplus_{i \geq 0} B[z] f \cdots f^{(i-1)} \subset \mathbb{H}[z] .
$$

Proposition 4.7. We have:

$$
\forall n \geq 1, \quad \operatorname{det}_{\mathbb{T}_{z}\left(\mathbb{K}_{\infty}\right)} \mathcal{L}(\varphi ; n ; z)=\prod_{\mathfrak{P}}\left(1-\frac{\rho\left(\left[\frac{B}{\mathfrak{P} B}\right]_{A}\right) z^{\operatorname{deg} N_{H / K}(\mathfrak{P})}}{\left[\frac{B}{\mathfrak{P} B}\right]_{A}^{n}}\right)^{-1} \in \mathbb{T}_{z}\left(\mathbb{K}_{\infty}\right)^{\times}
$$

where $\mathfrak{P}$ runs through the maximal ideals of $B$.

Proof. The proof is similar to that of Theorem 3.6. We give a sketch of the proof for the convenience of the reader.

Let $n \geq 1$. We have:

$$
\operatorname{det}_{\mathbb{T}_{z}\left(\mathbb{K}_{\infty}\right)} \mathcal{L}(\varphi ; n ; z)=\prod_{P} \operatorname{det}_{\mathbb{K}[z]}\left(1-\left.\frac{u_{P}}{\psi(P)^{n}} z^{\operatorname{deg} P} \sigma_{P}\right|_{\mathbb{H}[z]}\right)^{-1} .
$$

Let $P$ be a maximal ideal of $A$. Let $e \geq 1$ be the order of $P$ in $\operatorname{Pic}(A)$. Then $1, \sigma_{P}, \ldots, \sigma_{P}^{e-1}$ are linearly independent over $\mathbb{H}(z)$. We have :

$$
\left(\frac{u_{P}}{\psi(P)^{n}} z^{\operatorname{deg} P} \sigma_{P}\right)^{e}=\frac{\rho\left(\psi\left(P^{e}\right)\right) z^{e \operatorname{deg} P}}{\psi\left(P^{e}\right)^{n}} \in \mathbb{K}[z] .
$$


Thus the minimal polynomial of $\left.\frac{u_{P}}{\psi(P)^{n}} z^{\operatorname{deg} P} \sigma_{P}\right|_{\mathbb{H}(z)}$ over $\mathbb{K}(z)$ (and also over $\left.\mathbb{H}^{\left\langle\sigma_{P}\right\rangle}(z)\right)$ is equal to:

$$
X^{e}-\frac{\rho\left(\psi\left(P^{e}\right)\right) z^{e \operatorname{deg} P}}{\psi\left(P^{e}\right)^{n}} \in \mathbb{K}[z][X] .
$$

Therefore the characteristic polynomial of $\left.\frac{u_{P}}{\psi(P)^{n}} z^{\operatorname{deg} P} \sigma_{P}\right|_{\mathbb{H}(z)}$ over $\mathbb{K}(z)$ is equal to:

$$
\left(X^{e}-\frac{\rho\left(\psi\left(P^{e}\right)\right) z^{e \operatorname{deg} P}}{\psi\left(P^{e}\right)^{n}}\right)^{\frac{[H: K]}{e}} .
$$

One obtains the desired result by the same arguments as that used in the proof of Theorem 3.6 .

Remark 4.8. Let $L=\rho(K)\left(\mathbb{F}_{\infty}\right)\left(\left(q^{d_{\infty}}-1 \sqrt{-\pi}\right)\right)$, and let $\tau: L \rightarrow L$ be the continuous morphism of $\rho(K)$-algebras such that $\forall x \in \mathbb{F}_{\infty}\left(\left(q^{d \infty}-1 \sqrt{-\pi}\right)\right), \tau(x)=x^{q}$. Then there exists an element $\omega \in L^{\times}$(unique up to the multiplication of an element in $\left.\rho(K)^{\times}\right)$such that:

$$
\tau(\omega)=f \omega .
$$

This element is a generalization of the special function introduced by G. Anderson and D. Thakur in [3. The existence of this element (combined with the log-algebraicity theorem) gives new arithmetic informations on special values of $L$-series. We refer the interested reader to a forthcoming work of the authors.

\subsection{Stark units and several variable log-algebraicity theorem.}

We set:

$$
U(\widetilde{\varphi} / W(B[z]))=\left\{x \in \mathbb{T}_{z}\left(\mathbb{H}_{\infty}\right), \exp _{\widetilde{\varphi}}(x) \in W(B[z])\right\} .
$$

The following result is a twisted (by the shtuka function $f$ ) version of [1], Theorem 5.1.1. :

Theorem 4.9. We have:

$$
U(\widetilde{\varphi} / W(B[z]))=\mathcal{L}(\varphi ; 1 ; z) W(B[z]) .
$$

In particular,

$$
\exp _{\widetilde{\varphi}}(\mathcal{L}(\varphi ; 1 ; z) W(B[z])) \subset W(B[z]),
$$

Proof. The proof is similar to that of Theorem 3.8. We give a sketch of the proof for the convenience of the reader.

Observe that $\exp _{\widetilde{\varphi}}: \mathbb{H}[[z]] \rightarrow \mathbb{H}[[z]]$ is an isomorphism of $\mathbb{A}[[z]]$-modules. Furthermore, if we set:

$$
W(H[[z]])=\oplus_{i \geq 0} H[[z]] f \cdots f^{(i-1)},
$$

we get:

$$
\exp _{\widetilde{\varphi}}(W(H[[z]]))=W(H[[z]])
$$

Let:

$$
W(B[[z]])=\oplus_{i \geq 0} B[[z]] f \cdots f^{(i-1)} \subset \mathbb{H}[[z]] .
$$

Let $P$ be a maximal ideal of $A$. Let $W_{P}=S^{-1} W(B[[z]])$, where $S=A \backslash P$. Then:

$$
P W_{P}=\psi(P) W_{P} .
$$

By Lemma 3.7, we have:

$$
\exp _{\widetilde{\varphi}}\left(P W_{P}\right)=P W_{P} .
$$


If

$$
\phi_{P}=\sum_{i=0}^{\operatorname{deg} P} \phi_{P, i} \tau^{i}
$$

we set:

$$
\widetilde{\varphi}_{P}=\sum_{i=0}^{\operatorname{deg} P} \phi_{P, i} f \cdots f^{(i-1)} z^{i} \tau^{i}
$$

We have:

$$
\widetilde{\varphi}_{P} \exp _{\widetilde{\varphi}}=\exp _{\sigma_{P}} \psi(P)
$$

where:

$$
\exp _{\sigma_{P}}=\sum_{i \geq 0} \sigma_{P}\left(e_{i}(\phi)\right) f \cdots f^{(i-1)} z^{i} \tau^{i}
$$

Let's set:

$$
U\left(\widetilde{\varphi} / W_{P}\right)=\left\{x \in \mathbb{H}[[z]], \exp _{\widetilde{\phi}}(x) \in W_{P}\right\} \subset W(H[[z]]) .
$$

We have an isomorphism of $A[[z]]$-modules induced by $\exp _{\widetilde{\varphi}}$ :

$$
\frac{U\left(\widetilde{\varphi} / W_{P}\right)}{P W_{P}} \simeq \widetilde{\varphi}\left(\frac{W_{P}}{P W_{P}}\right) .
$$

Note that:

$$
\forall i \geq 0, \quad \sigma_{P}\left(f \cdots f^{(i-1)}\right) u_{P}=f \cdots f^{(i-1)} \tau^{i}\left(u_{P}\right) \in W(B) .
$$

Therefore:

$$
\left(\widetilde{\varphi}_{P}-z^{\operatorname{deg} P} u_{P} \sigma_{P}\right) \widetilde{\varphi}\left(\frac{W_{P}}{P W_{P}}\right)=\{0\}
$$

Since $u_{P}$ is a "P-unit", for $x \in W(H[[z]]) \backslash W_{P},\left(\widetilde{\varphi}_{P}-z^{\operatorname{deg} P} u_{P} \sigma_{P}\right)(x)$ is not $P$-integral as an element of $\mathbb{H}[[z]]$. Thus:

$$
\widetilde{\varphi}\left(\frac{W_{P}}{P W_{P}[[z]]}\right)=\left\{x \in \widetilde{\varphi}\left(\frac{W(H[[z]])}{P W_{P}}\right),\left(\widetilde{\varphi}_{P}-z^{\operatorname{deg} P} u_{P} \sigma_{P}\right)(x)=0\right\} .
$$

Let $x \in W(H[[z]])$, we deduce that:

$$
x \in U\left(\widetilde{\varphi} / W_{P}\right) \Leftrightarrow\left(\widetilde{\varphi}_{P}-z^{\operatorname{deg} P} u_{P} \sigma_{P}\right)\left(\exp _{\widetilde{\varphi}}(x)\right) \in P W_{P} .
$$

Thus:

$$
x \in U\left(\widetilde{\varphi} / W_{P}\right) \Leftrightarrow \exp _{\sigma_{P}}\left(\psi(P) x-z^{\operatorname{deg} P} u_{P} \sigma_{P}(x)\right) \in P W_{P} .
$$

Lemma 3.7 implies:

$$
x \in U\left(\widetilde{\varphi} / W_{P}\right) \Leftrightarrow \psi(P) x-z^{\operatorname{deg} P} u_{P} \sigma_{P}(x) \in P W_{P} .
$$

Thus:

$$
U\left(\tilde{\varphi} / W_{P}\right)=\left(1-\frac{z^{\operatorname{deg} P} u_{P}}{\psi(P)} \sigma_{P}\right)^{-1} W_{P} .
$$

Observe that $W(B[[z]])=\bigcap_{P} W_{P}$. We conclude that:

$$
W(B[[z]])=\exp _{\widetilde{\varphi}}(\mathcal{L}(\varphi ; 1 ; z) W(B[[z]])) .
$$

By Lemma 4.4, we get:

$$
\exp _{\widetilde{\varphi}}(\mathcal{L}(\varphi ; 1 ; z) W(B[z])) \subset \mathbb{T}_{z}\left(\mathbb{H}_{\infty}\right) \cap W(B[[z]])=W(B[z]) .
$$

Recall that:

$$
U(\widetilde{\varphi} / W(B[z]))=\left\{x \in \mathbb{T}_{z}\left(\mathbb{H}_{\infty}\right), \exp _{\widetilde{\varphi}}(x) \in W(B[z])\right\}
$$


Then:

$$
U(\widetilde{\varphi} / W(B[z]))=\mathcal{L}(\varphi ; 1 ; z) W(B[[z]]) \cap \mathbb{T}_{z}\left(\mathbb{H}_{\infty}\right) .
$$

But recall that:

$$
\mathcal{L}(\varphi ; 1 ; z) \in\left(\mathbb{T}_{z}\left(\mathbb{H}_{\infty}\right)[G]\right)^{\times}
$$

Thus:

$$
U(\widetilde{\varphi} / W(B[z]))=\mathcal{L}(\varphi ; 1 ; z) W(B[z])
$$

Let ev $: \mathbb{T}_{z}\left(\mathbb{H}_{\infty}\right) \rightarrow \mathbb{H}_{\infty}$ be the evaluation map at $z=1$. Then by Proposition 4.7. we get:

$$
L(\varphi / W(B))=\operatorname{det}_{\mathbb{K}_{\infty}} \operatorname{ev}(\mathcal{L}(\varphi ; 1 ; z))
$$

where:

$$
\operatorname{ev}(\mathcal{L}(\varphi ; 1 ; z))=\prod_{P}\left(1-\frac{u_{P}}{\psi(P)} \sigma_{P}\right)^{-1}=\sum_{I} \frac{u_{I}}{\psi(I)} \sigma_{I} \in\left(\mathbb{H}_{\infty}[G]\right)^{\times},
$$

where $I$ runs through the non-zero ideals of $A$. Furthermore, by the above Theorem:

$$
\exp _{\varphi}(\operatorname{ev}(\mathcal{L}(\varphi ; 1 ; z)) W(B)) \subset W(B)
$$

And also:

$$
\exp _{\varphi}(\operatorname{ev}(\mathcal{L}(\varphi ; 1 ; z)) W(B) \mathbb{B}) \subset W(B) \mathbb{B} .
$$

If we define the regulator of Stark units $\operatorname{ev}(\mathcal{L}(\varphi ; 1 ; z)) W(B) \mathbb{B}$ as follows:

$$
[W(B) \mathbb{B}: \operatorname{ev}(\mathcal{L}(\varphi ; 1 ; z)) W(B) \mathbb{B}]_{\mathbb{A}}:=\operatorname{det}_{\mathbb{K}_{\infty}} \operatorname{ev}(\mathcal{L}(\varphi ; 1 ; z)),
$$

then:

$$
L(\varphi / W(B))=[W(B) \mathbb{B}: \operatorname{ev}(\mathcal{L}(\varphi ; 1 ; z)) W(B) \mathbb{B}]_{\mathbb{A}} .
$$

We now briefly discuss the several variable version of Theorem 4.9. Let $s \geq 0$ be an integer. Let:

where:

$$
K_{s}=\operatorname{Frac}\left(A^{\otimes s}\right)
$$

$$
A^{\otimes s}=A \otimes_{\mathbb{F}_{q}} \cdots \otimes_{\mathbb{F}_{q}} A .
$$

If $s=0$, then $K_{0}=\mathbb{F}_{q}$. Let:

$$
\begin{aligned}
& \mathbb{H}_{s}=\operatorname{Frac}\left(A^{\otimes s} \otimes_{\mathbb{F}_{q}} B\right), \\
& \mathbb{K}_{s}=\operatorname{Frac}\left(A^{\otimes s} \otimes_{\mathbb{F}_{q}} A\right) .
\end{aligned}
$$

For $i=1, \ldots, s$, let:

$$
\rho_{i}: A \rightarrow \mathbb{H}_{s}, \quad a \mapsto(1 \otimes \cdots 1 \otimes a \otimes \cdots \otimes 1) \otimes 1,
$$

where $a$ appears at the $i$-th position. We still denote by $\rho_{i}: \mathbb{H} \rightarrow \mathbb{H}_{s}$ the homomorphism of $H$-algebras such that:

$$
\forall a \in A, \quad \rho_{i}(\rho(a))=\rho_{i}(a) .
$$

We view $\mathbb{H}_{s}$ and $\mathbb{K}_{s}$ as functions fields over $K_{s} \otimes 1$. Let $\infty$ be the unique place of $\mathbb{K}_{s} / K_{s} \otimes 1$ above the place $\infty$ of $K / \mathbb{F}_{q}$. Then:

$$
\mathbb{K}_{s, \infty}=\left(K_{s} \otimes 1\right)\left(\mathbb{F}_{\infty}\right)\left(\left(1^{\otimes s} \otimes \pi\right)\right),
$$

and we set:

$$
\mathbb{H}_{s, \infty}=\mathbb{H}_{s} \otimes_{\mathbb{K}_{s}} \mathbb{K}_{s, \infty}
$$


Let $\mathbb{T}_{z}\left(\mathbb{H}_{s, \infty}\right)$ be the Tate algebra in the variable $z$ with coefficients in $\mathbb{H}_{s, \infty}$. Let $\tau: \mathbb{T}_{z}\left(\mathbb{H}_{s, \infty}\right) \rightarrow \mathbb{T}_{z}\left(\mathbb{H}_{s, \infty}\right)$ be the continuous homomorphism of $\left(K_{s} \otimes 1\right)[z]$-algebras such that:

$$
\forall x \in H_{\infty}, \quad \tau(x)=x^{q} .
$$

Let's set:

$$
W_{s}(B[z])=\oplus_{i_{1}, \ldots i_{s} \geq 0} B[z] \prod_{j=1}^{s} \rho_{j}(f) \cdots \tau^{\left(i_{j}-1\right)}\left(\rho_{j}(f)\right) \subset \mathbb{H}_{s}[z] .
$$

In particular $W_{0}(B[z])=B[z]$. By similar arguments as those of the proof of Lemma 4.4, we show that $W_{s}(B[z])$ is discrete in $\mathbb{T}_{z}\left(\mathbb{H}_{s, \infty}\right)$. For $n \geq 1$, we set:

$$
\mathcal{L}\left(\varphi_{s} ; n ; z\right):=\prod_{P}\left(1-\frac{\prod_{j=1}^{s} \rho_{j}\left(u_{P}\right)}{\psi(P)^{n}} z^{\operatorname{deg} P} \sigma_{P}\right)^{-1} \in\left(\mathbb{T}_{z}\left(\mathbb{H}_{s, \infty}\right)[G]\right)^{\times},
$$

where $P$ runs through the maximal ideals of $A$. Then, by the same proof as that of Proposition 4.7 for all $n \geq 1$, we get:

$$
\operatorname{det}_{\mathbb{T}_{z}\left(\mathbb{K}_{s, \infty}\right)} \mathcal{L}\left(\varphi_{s} ; n ; z\right)=\prod_{\mathfrak{P}}\left(1-\frac{\left(\prod_{j=1}^{s} \rho_{j}\left(\left[\frac{B}{\mathfrak{P} B}\right]_{A}\right)\right) z^{\operatorname{deg} N_{H / K}(\mathfrak{P})}}{\left[\frac{B}{\mathfrak{P} B}\right]_{A}^{n}}\right)^{-1} \in \mathbb{T}_{z}\left(\mathbb{K}_{s, \infty}\right)^{\times},
$$

where $\mathfrak{P}$ runs through the maximal ideals of $B$.

We define:

$$
\exp _{\widetilde{\varphi}_{s}}=\sum_{i \geq 0} e_{i}(\phi)\left(\prod_{j=1}^{s} \rho_{j}(f) \cdots \tau^{i-1}\left(\rho_{j}(f)\right)\right) z^{i} \tau^{i} \in \mathbb{H}_{s}\{\{\tau\}\}
$$

Then $\exp _{\widetilde{\varphi}_{s}}$ converges on $\mathbb{T}_{s}\left(\mathbb{H}_{s, \infty}\right)$, and we set:

$$
U\left(\widetilde{\varphi}_{s} / W_{s}(B[z])\right)=\left\{x \in \mathbb{T}_{z}\left(\mathbb{H}_{s, \infty}\right), \exp _{\widetilde{\varphi}_{s}}(x) \in W_{s}(B[z])\right\} .
$$

By similar arguments as those of the proof of Theorem 4.9, we get:

Corollary 4.10. We have:

$$
U\left(\widetilde{\varphi}_{s} / W_{s}(B[z])\right)=\mathcal{L}\left(\varphi_{s} ; 1 ; z\right) W_{s}(B[z]) .
$$

Example 4.11. We consider the Carlitz example, where $g=0$ and $d_{\infty}=1$. Observe that there exists $\theta \in K$ such that $\operatorname{sgn}(\theta)=1$, and $A=\mathbb{F}_{q}[\theta]$. Thus, $K=\mathbb{F}_{q}(\theta)$, and $K_{\infty}=\mathbb{F}_{q}\left(\left(\frac{1}{\theta}\right)\right)$.

Let $\phi: A \rightarrow \bar{K}_{\infty}\{\tau\}$ be the Carlitz module defined by

$$
\phi_{\theta}=\theta+\tau \text {. }
$$

Then the Carlitz exponential is given by:

$$
\exp _{\phi}=\sum_{i \geq 0} \frac{1}{D_{i}} \tau^{i}
$$

where for $i \geq 0, D_{i}=\prod_{k=0}^{i-1}\left(\theta^{q^{i}}-\theta^{q^{k}}\right)$.

The Hilbert class field $H$ of $K$ is $K$, and then $B=A$. Then, the shtuka function $f \in K \otimes H$ associated to the Carlitz module via the Drinfeld correspondence is given by:

$$
f=\theta \otimes 1-1 \otimes \theta
$$

Let $s \geq 0$ be an integer. For $i=1, \ldots, s$, let $t_{i}=\rho_{i}(\theta)$. We have:

$$
\rho_{i}(f)=t_{i}-\theta,
$$




$$
\begin{gathered}
\mathbb{H}_{s}=\mathbb{K}_{s}=\mathbb{F}_{q}\left(t_{1}, \ldots, t_{s}, \theta\right), \\
\mathbb{H}_{s, \infty}=\mathbb{K}_{s, \infty}=\mathbb{F}_{q}\left(t_{1}, \ldots, t_{s}\right)\left(\left(\frac{1}{\theta}\right)\right) .
\end{gathered}
$$

For $i \geq 0, j=1, \cdots s$, set:

$$
b_{i}\left(t_{j}\right)=\prod_{k=0}^{i-1}\left(t_{j}-\theta^{q^{k}}\right)
$$

We get:

$$
W_{s}(B[z])=A\left[t_{1}, \ldots, t_{s}\right][z]
$$

Observe that:

$$
\exp _{\widetilde{\varphi}_{s}}=\sum_{i \geq 0} \frac{\prod_{j=1}^{s} b_{i}\left(t_{j}\right)}{D_{i}} \tau^{i}
$$

We have:

$$
\mathcal{L}\left(\varphi_{s} ; 1 ; z\right)=\sum_{a \in A_{+}} \frac{a\left(t_{1}\right) \cdots a\left(t_{s}\right)}{a} z^{\operatorname{deg}_{\theta} a},
$$

where $A_{+}$denotes the set of monic polynomials in $A=\mathbb{F}_{q}[\theta]$. In particular, for $s=1$, we recover the zeta function introduced by Pellarin [21].

Corollary 4.10 implies:

$$
\exp _{\widetilde{\varphi}_{s}}\left(\mathcal{L}\left(\varphi_{s} ; 1 ; z\right) A\left[t_{1}, \ldots, t_{s}, z\right]\right) \subset A\left[t_{1}, \ldots, t_{s}, z\right] .
$$

We refer the interested reader to [4, [6], [7, 9], for arithmetic applications of this latter result.

\subsection{Another proof of Anderson's log-algebraicity theorem.}

Corollary 4.12. Let $n \geq 0$ and let $X_{1}, \ldots, X_{n}, z$ be $n+1$ indeterminates over $K$.

Let $\tau: K\left[X_{1}, \ldots, X_{n}\right][[z]] \rightarrow K\left[X_{1}, \ldots, X_{n}\right][[z]]$ be the continuous $\mathbb{F}_{q}[[z]]$-algebra homomorphism for the $z$-adic topology such that $\forall x \in K\left[X_{1}, \ldots, X_{n}\right], \tau(x)=x^{q}$. Then:

$$
\forall b \in B, \quad \exp _{\widetilde{\phi}}\left(\sum_{I} \frac{\sigma_{I}(b)}{\psi(I)} \phi_{I}\left(X_{1}\right) \cdots \phi_{I}\left(X_{n}\right) z^{\operatorname{deg} I}\right) \in B\left[X_{1}, \ldots, X_{n}, z\right],
$$

where I runs through the non-zero ideals of A, and:

$$
\exp _{\widetilde{\phi}}=\sum_{i \geq 0} e_{i}(\phi) z^{i} \tau^{i}
$$

Proof. We first treat the case $n=0$. Let $b \in B$. By Theorem 4.9, we get:

$$
\forall k \geq 0, \quad \sum_{\operatorname{deg} I+i=k} e_{i}(\phi) f \cdots f^{(i-1)} \frac{\tau^{i}\left(u_{I} \sigma_{I}(b)\right)}{\psi(I)^{q^{i}}} \in W(B),
$$

and:

$$
\forall k \gg 0, \quad \sum_{\operatorname{deg} I+i=k} e_{i}(\phi) f \cdots f^{(i-1)} \frac{\tau^{i}\left(u_{I} \sigma_{I}(b)\right)}{\psi(I)^{q^{i}}}=0 .
$$

The coefficient of $f \cdots f^{(k-1)}$ in $\sum_{\operatorname{deg} I+i=k} e_{i}(\phi) f \cdots f^{(i-1)} \frac{\tau^{i}\left(u_{I} \sigma_{I}(b)\right)}{\psi(I)^{q^{i}}}$ is:

$$
\sum_{\operatorname{deg} I+i=k} e_{i}(\phi) \frac{\sigma_{I}(b)^{q^{i}}}{\psi(I)^{q^{i}}} .
$$


Therefore:

$$
\begin{aligned}
& \forall k \geq 0, \quad \sum_{\operatorname{deg} I+i=k} e_{i}(\phi) \frac{\sigma_{I}(b)^{q^{i}}}{\psi(I)^{q^{i}}} \in B . \\
& \forall k \gg 0, \quad \sum_{\operatorname{deg} I+i=k} e_{i}(\phi) \frac{\sigma_{I}(b)^{q^{i}}}{\psi(I)^{q^{i}}}=0 .
\end{aligned}
$$

Thus:

$$
\exp _{\widetilde{\phi}}\left(\sum_{I} \frac{\sigma_{I}(b)}{\psi(I)} z^{\operatorname{deg} I}\right) \in B[z] .
$$

We now assume that $n \geq 1$. We have an isomorphism of $B[z]$-modules

$$
\gamma: W(B[z]) \rightarrow \oplus_{i_{1}, \ldots, i_{n} \geq 0} B[z] X_{1}^{q^{i_{1}}} \cdots X_{n}^{q^{i_{n}}}
$$

such that:

$$
\forall i_{1}, \ldots, i_{n} \in \mathbb{N}, \quad \gamma\left(\prod_{j=1}^{n} \rho_{j}\left(f \cdots f^{\left(i_{j}-1\right)}\right)\right)=\prod_{j=1}^{n} X_{j}^{q^{i_{j}}} .
$$

Observe that:

$$
\gamma \circ \prod_{j=1}^{n} \rho_{j}(f) \tau=\tau \circ \gamma
$$

Furthermore:

$$
\gamma\left(\left(\prod_{j=1}^{n} \rho_{j}\left(u_{I}\right)\right)\right)=\phi_{I}\left(X_{1}\right) \cdots \phi_{I}\left(X_{n}\right)
$$

Thus, we get by Corollary 4.10

$$
\exp _{\widetilde{\varphi}_{n}}\left(\mathcal{L}\left(\varphi_{n} ; 1 ; z\right) b\right) \in W_{n}(B[z])
$$

and thus:

$$
\exp _{\tilde{\phi}}\left(\sum_{I} \frac{\sigma_{I}(b)}{\psi(I)} \phi_{I}\left(X_{1}\right) \cdots \phi_{I}\left(X_{n}\right) z^{\operatorname{deg} I}\right) \in \oplus_{i_{1}, \ldots i_{n} \geq 0} B[z] X_{1}^{q^{i_{1}}} \cdots X_{n}^{q^{i_{n}}}
$$

Remark 4.13. Let $s \geq 1$ be an integer and let $B\left\{\tau_{1}, \ldots, \tau_{s}\right\}$ be the non-commutative polynomial ring in the variables $\tau_{1}, \ldots, \tau_{s}$, such that:

$$
\begin{gathered}
\tau_{i} \tau_{j}=\tau_{j} \tau_{i}, \\
\forall b \in B, \forall n \geq 0, \quad \tau_{i}^{n} b=b^{q^{n}} \tau_{i} .
\end{gathered}
$$

For $i=1, \ldots, s$, we set:

$$
\forall a \in A, \quad \varphi_{i, a}=\sum_{j=0}^{\operatorname{deg} a} \phi_{a, j} \tau_{i}^{j} \in B\left\{\tau_{1}, \ldots, \tau_{s}\right\},
$$

and:

$$
\forall a \in A, \quad \varphi_{a}=\sum_{j=0}^{\operatorname{deg} a} \phi_{a, j} \tau^{j} \in B\left\{\tau_{1}, \ldots, \tau_{s}\right\},
$$

where $\tau=\tau_{1} \cdots \tau_{s}$. 
Let $W_{s}(B)=\oplus_{i_{1}, \ldots, i_{s}} B \prod_{j=1}^{s} \rho_{j}(f) \cdots \tau^{i_{j}-1}\left(\rho_{j}(f)\right) \subset \mathbb{H}_{s}$. Then $W_{s}(B)$ is an $A^{\otimes s} \otimes B$-module. Let $j \in\{1, \ldots s\}$. Let $a \in A$, we have a natural $B$-module homomorphism:

such that:

$$
\widetilde{\rho}_{j}(a): B\left\{\tau_{1}, \ldots, \tau_{s}\right\} \rightarrow B\left\{\tau_{1}, \ldots, \tau_{s}\right\}
$$

$$
\forall i_{1}, \ldots, i_{s} \in \mathbb{N}, \quad \widetilde{\rho}_{j}(a) \cdot\left(\tau_{1}^{i_{1}} \cdots \tau_{s}^{i_{s}}\right)=\left(\tau_{j}^{i_{j}} \varphi_{j, a}\right) \prod_{k=1, k \neq j}^{s} \tau_{k}^{i_{k}} .
$$

Observe that:

$$
\forall i, j \in\{1, \ldots s\}, \forall a, b \in A, \quad \widetilde{\rho}_{j}(a) \circ \widetilde{\rho}_{i}(b)=\widetilde{\rho}_{i}(b) \circ \widetilde{\rho}_{j}(a) .
$$

Thus $B\left\{\tau_{1}, \ldots, \tau_{s}\right\}$ becomes an $A^{\otimes s} \otimes B$-module via:

$$
\forall x \in B\left\{\tau_{1}, \ldots, \tau_{s}\right\}, \quad\left(\sum_{i} b_{i} \prod_{j=1}^{s} \rho_{j}\left(a_{i, j}\right)\right) \cdot x=\sum_{i} b_{i}\left(\prod_{j=1}^{s} \tilde{\rho}_{j}\left(a_{i, j}\right)\right)(x) .
$$

Then, by the proof of Corollary 4.12, we have an $A^{\otimes s} \otimes B$-module isomorphism:

$$
B\left\{\tau_{1}, \ldots, \tau_{s}\right\} \simeq W_{s}(B) .
$$

In particular, $B\left\{\tau_{1}, \ldots, \tau_{s}\right\}$ is a finitely generated $A^{\otimes s} \otimes B$-module of rank one. The case $s=1$ was already observed by G. Anderson ([19], page 230, line 21 - there is a misprint in line 24 , since in general $f \notin A \otimes_{\mathbb{F}_{q}} \mathbb{C}_{\infty}$ ). If $I$ is a non-zero ideal of $A$, we define $I * \cdot: B\left\{\tau_{1}, \ldots, \tau_{s}\right\} \rightarrow B\left\{\tau_{1}, \ldots, \tau_{s}\right\}$ to be the $B$-module homomorphism such that:

$$
I *\left(\tau_{1}^{i_{1}} \cdots \tau_{s}^{i_{s}}\right)=\sum_{j_{1}, \ldots, j_{s} \in\{0, \ldots, \operatorname{deg} I\}} \phi_{I, j_{1}}^{q^{i_{1}}} \cdots \phi_{I, j_{s}}^{q^{i_{s}}} \tau_{1}^{i_{1}+j_{1}} \cdots \tau_{s}^{i_{s}+j_{s}},
$$

where $\phi_{I}=\sum_{k=0}^{\operatorname{deg} I} \phi_{I, k} \tau^{k}$.

Let $\mathcal{L}: B\left\{\tau_{1}, \ldots, \tau_{s}\right\} \rightarrow H\left\{\left\{\tau_{1}, \ldots, \tau_{s}\right\}\right\}$ be defined as follows:

$$
\mathcal{L}\left(\sum_{i_{1}, \ldots i_{s}} b_{i_{1}, \ldots, i_{s}} \tau_{1}^{i_{1}} \cdots \tau_{s}^{i_{s}}\right)=\sum_{i_{1}, \ldots, i_{s}} \sum_{I} \frac{\sigma_{I}\left(b_{i_{1}, \ldots, i_{s}}\right)}{\psi(I)} I *\left(\tau_{1}^{i_{1}} \cdots \tau_{s}^{i_{s}}\right)
$$

Then by Corollary 4.10, we get that the multiplication by $\exp _{\phi} \in H\{\{\tau\}\}$ on $H\left\{\left\{\tau_{1}, \ldots, \tau_{s}\right\}\right\}$ yields to the following property:

$$
\forall x \in B\left\{\tau_{1}, \ldots, \tau_{s}\right\}, \quad \exp _{\phi}(\mathcal{L}(x)) \in B\left\{\tau_{1}, \ldots, \tau_{s}\right\} .
$$

\section{REFERENCES}

[1] G. Anderson, Rank one elliptic A-modules and A-harmonic series, Duke Mathematical Journal 73 (1994), 491-542.

[2] G. Anderson, Log-Algebraicity of Twisted $A$-Harmonic Series and Special Values of $L$-series in Characteristic $p$, Journal of Number Theory 60 (1996), 165-209.

[3] G. Anderson, D. Thakur, Tensor powers of the Carlitz module and zeta values, Annals of Mathematics 132 (1990), 159-191.

[4] B. Anglès, T. Ngo Dac, F. Tavares Ribeiro, Exceptional Zeros of $L$-series and BernoulliCarlitz Numbers (with an appendix by B. Anglès, D. Goss, F. Pellarin, F. Tavares Ribeiro), arXiv: 1511.06209 (2015).

[5] B. Anglès, T. Ngo Dac, F. Tavares Ribeiro, Twisted Characteristic $p$ Zeta Functions, to appear in Journal of Number Theory, arXiv:1603.04076

[6] B. Anglès, F. Pellarin, F. Tavares Ribeiro, with an appendix by F. Demeslay, Arithmetic of positive characteristic $L$-series values in Tate algebras, Compositio Mathematica 152 (2016), $1-61$. 
[7] B. Anglès, F. Pellarin, F. Tavares Ribeiro, Anderson-Stark Units for $\mathbb{F}_{q}[\theta]$, to appear in Transactions of the American Mathematical Society, arXiv: 1501.06804.

[8] B. Anglès, L. Taelman, with an appendix by V. Bosser, Arithmetic of characteristic $p$ special $L$-values, Proceedings of the London Mathematical Society 110 (2015), 1000-1032.

[9] B. Anglès, F. Tavares Ribeiro, Arithmetic of function fields units, to appear in Mathematische Annalen, DOI 10.1007/s00208-016-1405-2.

[10] L. Carlitz, On certain functions connected with polynomials in Galois field, Duke Mathematical Journal 1 (1935), 137-168.

[11] F. Demeslay, A class formula for $L$-series in positive characteristic, arXiv:1412.3704 (2014).

[12] V. G. Drinfeld, Elliptic modules, Mathematics of the USSR-Sbornik 23 (English transl.) (1976), 561-592.

[13] V. G. Drinfeld, Commutative subrings of some noncommutative rings, Functional Analysis and its Applications 11 (1977), 11-14.

[14] V. G. Drinfeld, Varieties of modules of F-sheaves, Functional Analysis and its Applications 21 (English transl.) (1987), 107-122.

[15] J. Fang, Special L-values of abelian t-modules, Journal of Number Theory 147 (2015), 300325.

[16] J. Fang, Equivariant Special $L$-values of abelian $t$-modules, arXiv: 1503.07243 (2015).

[17] J. Fang, Equivariant trace formula mod p, Comptes Rendus Mathématique 354 (2016), 335338.

[18] E.-U. Gekeler, On Finite Drinfeld Modules, Journal of Algebra 141 (1991), 187-203.

[19] D. Goss, Basic Structures of Function Field Arithmetic, Springer, 1996.

[20] M. Papanikolas, Log-Algebraicity on Tensor Powers of the Carlitz Module and Special Values of Goss $L$-Functions, in preparation.

[21] F. Pellarin, Values of certain $L$-series in positive characteristic, Annals of Mathematics $\mathbf{1 7 6}$ (2012), 2055-2093

[22] L. Taelman, A Dirichlet unit theorem for Drinfeld modules, Mathematische Annalen 348 (2010), 899-907.

[23] L. Taelman, Special L-values of Drinfeld modules, Annals of Mathematics 175 (2012), 369391.

[24] J. Tate, On the conjectures of Birch and Swinnerton-Dyer and a geometric analog, Séminaire N. Bourbaki, 1964-1966, exp. no. 306, 415-440.

[25] J. Tate, Les Conjectures de Stark sur les Fonctions $L$ d'Artin en $s=0$, Birkhäuser, 1984.

[26] D. Thakur, Drinfeld modules and arithmetic in function fields, Int. Math. Res. Notices 9 (1992), 185-197.

[27] D. Thakur, Shtukas and Jacobi sums, Inventiones mathematicae 111 (1993), 557-570.

[28] D. Thakur, Function Field Arithmetic, World Scientific, 2004.

Normandie Université, Université de Caen Normandie, Laboratoire de Mathématiques Nicolas Oresme, CNRS UMR 6139, Campus II, Boulevard Maréchal Juin, B.P. 5186, 14032 Caen Cedex, France.

E-mail address: bruno.angles@unicaen.fr,tuan.ngodac@unicaen.fr,floric.tavares-ribeiro@unicaen.fr 